\title{
uPTI: uniaxial permittivity tensor imaging of intrinsic density and anisotropy
}

\author{
Li-Hao Yeh ${ }^{1, \bigotimes}$, Ivan E. Ivanov $^{1}$, Bryant B. Chhun ${ }^{1}$, Syuan-Ming Guo ${ }^{1}$, Ezzat Hashemi ${ }^{2}$, Janie R. Byrum ${ }^{1}$, Juan A. \\ Pérez-Bermejo ${ }^{3}$, Huijun Wang ${ }^{4}$, Yanhao Yu ${ }^{4}$, Peter G. Kazansky ${ }^{4}$, Bruce R. Conklin ${ }^{3,5}$, May H. Han ${ }^{2}$, and Shalin B. Mehta ${ }^{1, \mathbb{}}$ \\ ${ }^{1}$ Chan Zuckerberg Biohub, San Francisco, CA, USA \\ ${ }^{2}$ Stanford University, Palo Alto, CA, USA \\ ${ }^{3}$ Gladstone Institutes, San Francisco, CA, USA \\ ${ }^{4}$ University of Southampton, Southampton, UK \\ ${ }^{5}$ University of California San Francisco, CA, USA \\ ${ }^{\square}$ correspondence: lihao.yeh@czbiohub.org and shalin.mehta@czbiohub.org
}

\begin{abstract}
Architecture of biological systems is important for their homeostatic functions and is predictive of pathology. Volumetric imaging of intrinsic density, anisotropy, and 3D orientation of cell and tissue components is informative, but still challenging. These physical properties can be described succinctly by the volumetric distribution of the specimen's permittivity tensor (PT). We report uniaxial permittivity tensor imaging (uPTI), a novel approach for label-free volumetric imaging with diffraction-limited resolution. uPTI combines the oblique illumination and the polarization imaging with high numerical apertures to encode the specimen's permittivity tensor into intensity modulations, which are decoded with a novel vector diffraction model and a multichannel convex optimization. The uPTI volumes of polystyrene beads and laser-fabricated anisotropic glass targets show that 3D uPT measurements are quantitative, with diffraction-limited spatial resolution of $0.23 \times 0.23 \times 0.8 \mu \mathrm{m}^{3}$. Automated 2D and 3D imaging of a mouse brain section reveals anatomy at multiple scales $(\mathrm{cm}-250 \mathrm{~nm})$ and demonstrates that uPTI enables analysis of the density, anisotropy, and orientation of axon bundles and single axons. We multiplex uPTI with fluorescence deconvolution microscopy to enable correlative analysis of physical and molecular architecture of iPSC-derived cardiomyocytes. uPTI can be added to an existing widefield microscope as a low cost module. We share our implementation of the forward model and optimization algorithms via open source repository. Collectively, the reported advances in optical design, image formation, reconstruction algorithms, and biological interpretation open multiple avenues to study architecture of organelles, cells and tissue sections, including human cells and tissues that are challenging to label.
\end{abstract}

Label-free Microscopy | Inverse algorithms | Density | Anisotropy | High Resolution | Myelination | Organelle architecture

\section{Introduction}

Label-free imaging of architecture of biological systems at the scales ranging from molecules to whole body has enabled multiple discoveries. Electron microscopy (EM), which reports the distribution of charge density in fixed specimens, routinely provides structural insights with spatial resolution of around a nanometer, but requires more than 16 months and systematically optimized pipelines (tissue screening, tissue sectioning, automated acquisition, volume assembly) to image $1 \mathrm{~mm}^{3}$ sized tissue (1). Magnetic resonance imaging (MRI), which reports the distribution of hydrogen density, can image dynamic architecture at the scale of organs deep into body, but requires custom-built coils to achieve resolution of $100 \mu \mathrm{m}$ (2). A variant of MRI, the diffusion weighted MRI, reports not just the density, but also the anisotropy of hydrogen (or water) diffusion, described by a diffusion tensor. Diffusion tensor imaging (DTI) has been instrumental in analyzing connectivity of white matter in brains. Similarly, comprehensive measurement of intrinsic architecture at the spatial scales of organelles and cells can provide new insights in biology and pathology. Corresponding spatial scales $(250 \mathrm{~nm}-1 \mathrm{~mm})$ can be analyzed in live specimens with visible wavelength light microscopy. Label-free light microscopy probes the density and anisotropy of bound electrons described by the permittivity tensor and bridges the resolution gap between EM and MRI. It can enable new investigations, for example, rapid analysis of architecture of mammalian brains and other organs, response of diverse cell types to infection or other perturbations, and identification of types and states of human cells in a clinical sample.

Macromolecular components of biological systems (nucleic acids, amino acids, lipids, and glycans) are dielectric at visible wavelengths, i.e., the electrons bound to macromolecules conduct electricity poorly, but oscillate in response to applied electric field and modify the incident electric field. The spatio-angular architecture of biological systems is described by spatial distribution of the permittivity tensor (3), which quantifies local angular distribution of bound electrons. To be more specific, the relative permittivity is a measure of how easily bound electrons in a material are polarized ${ }^{1}$ by an applied electric field. The more easily a material is polarized, the more it delays the electromagnetic wave travelling through it, and the more optically dense the material is. If the bound electrons resonate with the incident optical frequency, the material absorbs the light. At

\footnotetext{
${ }^{1}$ the polarization here refers to transient changes in charge distribution and not the plane of oscillation of electric field vector.
} 
visible wavelengths, biological specimens typically induce substantial phase delay, but negligible absorption, which makes them transparent. The permittivity of an isotropic material formed by disordered molecules, such as water, is a scalar independent of the direction and the polarization of the incident light. The permittivity of an anisotropic material formed by aligned molecules, such as collagen or lipid bi-layer, is a tensor that depends on the direction and polarization of the incident light (3). The permittivity tensor of a complex specimen can be decomposed into the isotropic component that reports the local density of macromolecules and the anisotropic component that reports the local angular distribution of macromolecules.

Majority of the label-free light microscopy methods assume that biological material is isotropic or is anisotropic with a single axis of symmetry. These assumptions amount to the measurement of the isotropic or anisotropic components of the uniaxial permittivity tensor (uPT). For example, quantitative phase microscopy images the isotropic component of uPT by measuring the density (optical path length) and has been used to analyze membrane mechanics (4), density and dynamics of cell organelles $(5,6)$, urine cytology (7), etc. Similarly, quantitative polarization microscopy images the anisotropic component of permittivity tensor (the retardance and the orientation) and has been used to study microtubule spindle $(8,9)$, white matter in human brain tissue slices (10-15), collagen architecture in the eye tissues $(16,17)$, etc. Both of these approaches are also used to quantify the optical properties of the fabricated materials $(18,19)$. Even though the isotropic and anisotropic components of uPT are measured separately, these contrast are generated simultaneously when light scatters from the specimen. Quantitative phase imaging methods that account for effects of diffraction can achieve spatial resolution of $0.2 \times 0.2 \times 0.4 \mu \mathrm{m}^{3}$ (Nanolive (20) and Tomocube (21)) and $0.3 \times 0.3 \times 1.1 \mu \mathrm{m}^{3}$ (GLIM (22)). However, majority of polarization imaging methods do not account for the effects of light diffraction in reconstruction and therefore do not achieve diffraction-limited spatial resolution. Hence, a method and a diffraction model that combine these approaches for complete measurement of uPT can improve the accuracy and the resolution of label-free microscopy.

In recent years, a few approaches for simultaneous measurement of the density and the anisotropy have been developed. Shribak et al. reported a method combining orientation-independent differential interference contrast microscopy (OI-DIC) and quantitative polarization microscopy that provides joint density and 2D anisotropy (retardance and orientation projected on the image plane) measurements in a $2 \mathrm{D}$ plane $^{2}$, without considering diffraction effects (23). Ptychography-based phase retrieval method has been extended with polarization sensitive components and Jones calculus for joint imaging of density and 3D anisotropy (principal retardance and 3D orientation) in a 2D plane considering diffraction $(24,25)$. Our recent work, quantitative label-free imaging with phase and polarization (QLIPP), combines phase from defocus (26-28) with quantitative polarization micorscopy (29-31) to measure density and 2D anisotropy in 3D space (32). Although these methods retrieve more information than traditional phase and polarization microscopy, they do not measure uniaxial permittivity tensor with diffraction-limited resolution, which can enable new studies with a light microscope, just as diffusion tensor imaging enabled new studies with MRI.

We report uniaxial permittivity tensor imaging (uPTI), a computational microscopy technique, which provides diffractionaware measurements of absorption, phase, principal retardance, 3D orientation, and symmetry in 2D and 3D space. uPTI captures these properties of the specimen by combining oblique illumination (33-36) with polarization-sensitive detection (10, $30,37-42)$. We implement this design on a research microscope with inexpensive add-on modules. We develop a vectorial partially coherent imaging model and the corresponding multi-channel deconvolution algorithm to extract spatial distribution of the components of the uniaxial permittivity tensor. We validate the accuracy and resolution with isotropic polystyrene beads and anisotropic laser-written glass targets. We demonstrate that uPTI allows analysis of the architecture of the mouse brain at scales of whole slice, axon bundles, and single axons. Finally, we show that uPTI measurements can be multiplexed with fluorescence deconvolution microscopy to enable analysis of the physical and molecular architecture of organelles in iPSCderived cardiomyocytes. These data establish a novel label-free measurement technology for comprehensive high-resolution imaging of biological architecture. With our inexpensive optical design and open source software, we aim to enable rapid adoption and refinement.

\section{Results}

\section{A: Imaging of uniaxial permittivity tensor}

The spatio-angular distribution of bound electrons (i.e. the permittivity tensor of the material) determines how specimen interacts with light and depends on the distribution of dry mass and material anisotropy. In this work, we assume that the material anisotropy has single axis of symmetry, i.e. the material is uniaxial. We illuminate the specimen from diverse directions and probe the scattered light with diverse polarizations to convert invisible volumetric distribution of uPT into visible volumetric distribution of intensities.

\section{Light path}

Figure 1A and Figure 1-supplement 1 show the optical setup of uPTI. We implemented uPTI on a Leica DMi8 inverted microscope with two add-on modules, the oblique illuminator and the polarization imaging module. The oblique illuminator is

\footnotetext{
${ }^{2}$ Since we are referring to spatio-angular coordinates, $2 \mathrm{D} / 3 \mathrm{D}$ anisotropy and orientation should be assumed to be angular dimensions, whereas $2 \mathrm{D}$ plane/3D volume should be assumed to be spatial dimensions.
} 
bioRxiv preprint doi: https://doi.org/10.1101/2020.12.15.422951; this version posted December 16, 2020. The copyright holder for this preprint (which was not certified by peer review) is the author/funder, who has granted bioRxiv a license to display the preprint in perpetuity. It is made available under aCC-BY-NC-ND 4.0 International license.

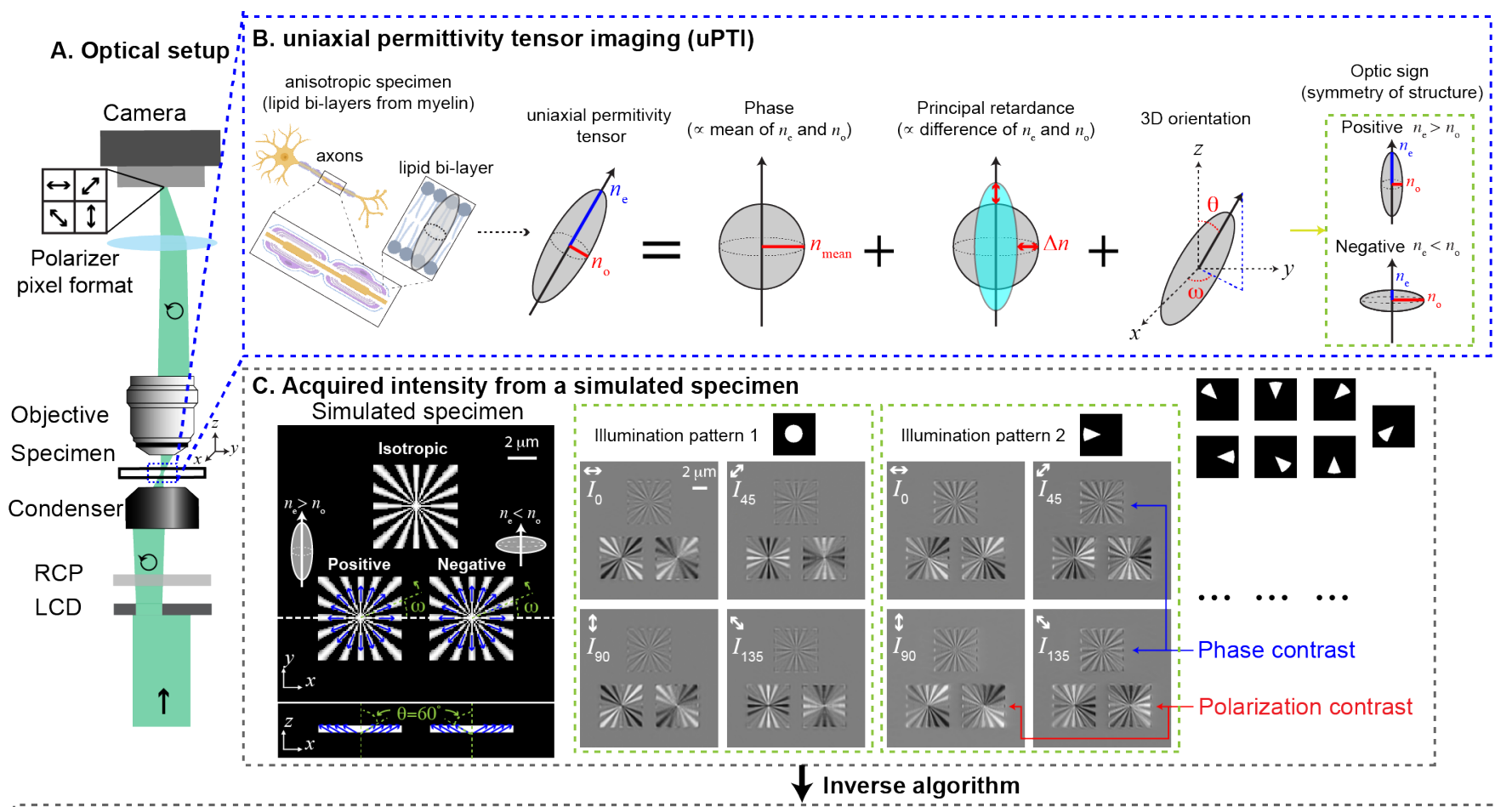

D. Reconstructed simulated specimen
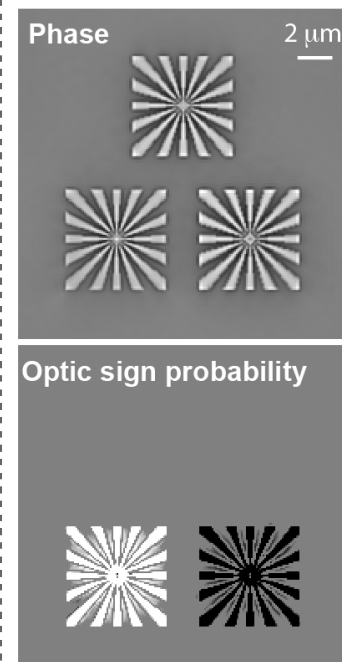
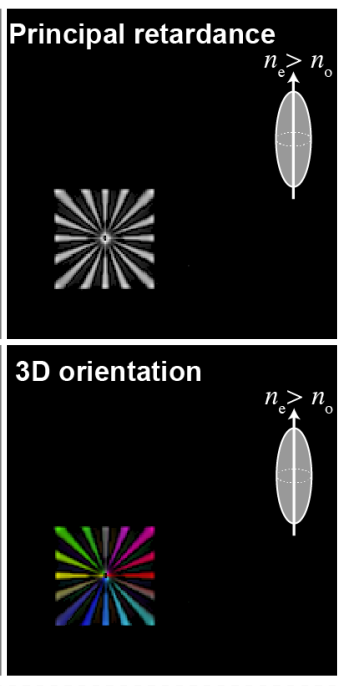

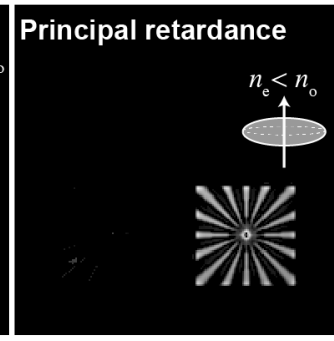

3D orientation

Fig. 1. Measurements with the uniaxial permittivity tensor imaging (uPTI): (A) Light path of the microscope. (B) Optical anisotropy arises from the angular anisotropy of biological architecture, e.g., ordered lipid bilayers in the myelinated axons give rise to a higher refractive index perpendicular to the bilayer. The optical anisotropy can be represented by a uniaxial permittivity tensor (UPT) or an indicatrix, which is an ellipsoidal surface that represents angular distribution of the optical path length over the spatial resolution of the imaging system. The uPT is parameterized by its 3D orientation $(\omega$ and $\theta)$ and two principal axes $\left(n_{\mathrm{e}}\right.$ and $\left.n_{\mathrm{O}}\right)$. The uPT can be separated and interpreted in terms of four physical quantities: the average delay in the light induced by the specimen (phase), the degree of anisotropy (principal retardance), 3D orientation, and the symmetry of the structure (optic sign, $n_{\mathrm{e}} \gtrless n_{\mathrm{o}}$ ). (C) Simulated raw images of a structure consisting of isotropic material, positive uniaxial material, and negative uniaxial material oriented radially (along the spokes) and inclined with $\theta=60^{\circ}$ illustrate dependence of intensity contrast on the polarization and the angle of illumination. We observe intensity modulations due to specimen properties in the simulated intensities. Phase variations are evident in isotropic material with off-axis illumination (illumination pattern 2), while anisotropy variations are better observed in different polarization channels. We also observe contrast variations across on-axis and off-axis illumination due to variations in out-of-plane orientation and optic sign. (D) Using an inverse algorithm based on convex optimization, we reconstruct phase, principal retardance, optic sign probability, and 3D orientation of the simulated target from intensities. The 3D orientation image shows false-color images in which the 3D orientations is shown by the color and the principal retardance is shown by the brightness of the color. We assign a color to the $3 \mathrm{D}$ orientation using a spherical colormap adopted from (43) (e.g. $\omega=0^{\circ}$ and $\theta=45^{\circ}$ for red, $\omega=180^{\circ}$ and $\theta=45^{\circ}$ for yellow) and shown in the last column. We report the $3 \mathrm{D}$ orientation of the symmetry axis of the permittivity tensor, independent of its optic sign. 
composed of a green color filter, a linear polarizer, an amplitude modulator, a right-hand circular polarizer, and a condenser lens. The light from an LED source is first filtered by a green filter and the linear polarizer before passing through the amplitude modulator placed in the front focal plane of the condenser lens. The amplitude modulator is constructed from a low-cost liquid crystal display (LCD, Adafruit, ST7735R) (36). The right-hand circular polarizer (RCP, Thorlabs, CP1R532) is placed after the amplitude modulator. This module enables computer-controlled oblique illumination with circular polarized light. It is compact enough to be placed close to the front focal plane of the high-NA condenser and allows illumnation of the specimen with the numerical aperture (NA) as high as 1.4 with good light coupling efficiency. The oblique circular polarized light then impinges on the specimen, generating scattered light going into the polarization imaging module. The polarization imaging module is formed by the microscope objective, tube lens, and a four-channel polarization sensitive camera (FLIR, BFS-U351S5P-C). The polarization sensitive camera has a grid of linear polarizers of $0^{\circ}, 45^{\circ}, 90^{\circ}, 135^{\circ}$ on top of the sensor and images four linearly polarized light states with a single exposure. A microscope with this camera can capture the projected retardance and 2D orientation of material's slow axis similar to other polarized light microscopes $(10,31,32,37,42)$. The module that enables acquisition of 3D anisotropy is the high-quality oblique illuminator. Overall, our modular design enables tomographic polarization imaging of specimens with diverse oblique illumination patterns.

\section{Interpretation of UPT}

Figure 1B illustrates how 3D anisotropy of a lipid bi-layer structure is described by a uniaxial permittivity tensor (uPT) or an indicatrix, an ellipsoidal surface parameterized by 3D orientation $(\omega$ and $\theta)$ of the symmetry axis, the ordinary refractive index $\left(n_{\mathrm{o}}\right)$ experienced by the waves with the electric field in the directions perpendicular to the symmetry axis, and the extraordinary refractive index $\left(n_{\mathrm{e}}\right)$ experienced by the waves with the electric field in the direction parallel to the symmetry axis. The uPT can be interpreted through four physical components: 1. average delay in the light induced by the specimen (phase proportional to the mean of $n_{\mathrm{e}}$ and $n_{\mathrm{o}}$ ), 2. degree of anisotropy (principal retardance proportional to the difference of $n_{\mathrm{e}}$ and $n_{\mathrm{o}}$ ), 3. 3D orientation of the symmetry axis or optic axis of the material (azimuthal angle $\omega$ and inclination $\theta$ ), and 4. symmetry of the structure around the optic axis (optic sign, $n_{\mathrm{e}} \gtrless n_{\mathrm{o}}$ ). Materials are referred to as positive or negative uniaxial depending on their optic sign (3). For example, the spatially resolved lipid bi-layer is a positive uniaxial material, whereas the anisotropy of the test target ( fig. 2) and the whole axons reported later in fig. 4 are negative uniaxial. To draw an analogy to the diffusion tensor imaging (DTI), the phase component of uPT is similar to the mean diffusivity component of DT, the principal retardance component of uPT is similar to the fractional anisotropy component of DT, and the 3D orientation of anisotropy of uPT is similar to the 3D orientation of the axial diffusivity of DT (44). The imaging resolution and depth of DTI and uPTI, however, is quite different. DTI is suitable for organ to tissue level of imaging deep inside the specimen's body, while uPTI is suitable for organelle, cell, and tissue level of imaging up to the depth of few tens of microns.

\section{Image acquisition and simulation}

To measure the uPT distribution, we image our specimen with diverse oblique illuminations and four linear polarization states. The choice of the illumination patterns projected on the LCD is discussed in Methods. We illustrate the transfer of material properties to intensities and reconstruction of material properties with a simulation in fig. 1C. We show raw images of a structure consisting of isotropic material $\left(n_{\mathrm{e}}=n_{\mathrm{o}}=1.52\right)$, positive uniaxial material $\left(n_{\mathrm{e}}=1.521, n_{\mathrm{o}}=1.519\right)$, and negative uniaxial material $\left(n_{\mathrm{e}}=1.519, n_{\mathrm{o}}=1.521\right)$ with the optic axes oriented at $\omega$ aligned radially (along the spokes) and inclined with $\theta=60^{\circ}$. The refractive index of the surrounding medium is set to 1.518 . Each type of material is $5.7 \times 5.7 \mu \mathrm{m}^{2}$ in size. We set the NA of illumination to be 1.4 and the NA of the objective to be 1.47 to mimic the experimental conditions of the later high-resolution imaging (fig. 2, 3C-3I, 4, 5). When a circular illumination pattern (illumination pattern 1) is displayed on the LCD, which corresponds to brightfield illumination, we see almost no contrast for the isotropic material and strong intensity modulations due to the anisotropic materials in four polarization channels. When the illumination pattern is a sector (illumination pattern 2), edges of both isotropic and anisotropic materials are highlighted, which is a demonstration of differential phase contrast introduced by oblique illumination (33-35). We also observe that the two anisotropic materials cause differential intensity modulations in the four polarization channels when the on-axis (brightfield) and the off-axis (sector) illuminations are used. This is due to the difference in the optic sign and the 3D orientation of optic axis of the material (38-40). The simulation so far is done for a 2D specimen. If the specimen is 3D in nature, i.e., the thickness of the specimen is larger than the depth of field of the microscope, instead of collecting intensity in a 2D plane, we collect a $z$-stack of four polarization channels for each of the nine oblique illumination patterns. In summary, we collect 36 ( 9 oblique illuminations and 4 polarization channels) 2D images or 3D $z$-stacks depending on whether the specimen is thinner than the depth of field. To account for background polarization effects introduced by components in the optical path other than the specimen, we also collect a dataset (36 2D images from 9 oblique illuminations and 4 polarization channels) at an empty field of view, which is used in the reconstruction of the physical properties of the specimen (see Methods).

\section{Reconstruction of UPT}

Extraction of components of uPT from measured intensities considering diffraction requires a vectorial partially coherent imaging model that expresses intensities in terms of the components of uPT. We develop and summarize this model in Methods 
which expresses a uPT as a scattering potential tensor and maps the scattering potential tensor to the Stokes parameters measured in the image plane. Scalar scattering potential is a scaled difference of the refractive indices of the specimen and the surrounding medium, which is a key concept employed in the diffraction tomography of 3D refractive index (density of bound electrons) (45). An extension of scattering potential, scattering matrix, has been proposed (46) for volumetric reconstruction of projected anisotropy, but not experimentally verified. Our work extends these concepts and introduces the scattering potential tensor, which allows reconstruction of volumetric distribution of density, 3D anisotropy, and material symmetry. The mapping between scattering potential tensor and intensities recorded by uPTI is nonlinear. The nonlinearity of the mapping makes the inverse problem challenging, because it can create ambiguities when searching for a unique solution. Fortunately, this mapping can be linearized when the scattering is weak enough such that the single-scattered photons contribute to the most of intensity variations. With this approximation, which corresponds to the first Born and weak object approximation (3), we build a forward model. Our forward model describes a linear relationship between the Stokes parameters and the scattering potential tensor via the transfer functions that depend on the parameters of the microscope. This forward model then serves as the theoretical foundation to develop a deconvolution algorithm that retrieves the UPT from high-dimensional acquisition. Before starting to reconstruct the components of the uPT, we first calibrate the mapping between Stokes parameters and the measured intensities (47), convert the measured intensities into the Stokes parameters, and do a background correction. The deconvolution algorithm (Methods) converts the Stokes parameters into physical properties summarized in fig. 1B. This computational framework allows us to transform the input intensities from fig. 1C into diffraction limited measurements of phase, principal retardance, optic sign probability, and 3D orientation of the specimen as shown in fig. 1D. The python software that implements the forward model, the reconstruction algorithm, the simulations, and illustration of reconstruction are available at https://github.com/mehta-lab/waveorder.

\section{B: Accuracy and spatial resolution of UPTI}

As illustrated in fig. 1, uPTI provides four distinct volumetric measurements: phase, principal retardance, 3D orientation, and the optic sign. To validate the accuracy and diffraction-limited volumetric resolution achievable by uPTI for these channels of information, we image three types of isotropic and anisotropic test targets. Each type of target characterizes different aspects of the method. uPTI is designed to use high NA partially coherent illumination and high NA imaging, which enables confocal-like depth sectioning in measured channels. All volumes reported in this result are acquired with an $1.4 \mathrm{NA}\left(\mathrm{NA}_{\mathrm{c}}\right)$ oil immersion condenser and 1.47NA $\left(\mathrm{NA}_{\mathrm{o}}\right)$ oil immersion objective.

\section{Accuracy}

We first characterize the accuracy of phase and principal retardance by imaging isotropic $3 \mu \mathrm{m}$ polystyrene beads embedded in oils of varied refractive indices as shown in fig. 2A. The refractive index (RI) of the polystyrene beads is known to be $n_{\text {beads }}=1.5956$ at wavelength of $532 \mathrm{~nm}$. Embedding the beads in the media of varying RI (from 1.5536 to 1.5826 ) changes the accumulated optical path length (theoretical phase) of the light as well as the amount of edge retardance (48) linearly. When the RI of the surrounding media is the same as the refractive index of the beads, there will be no accumulated phase and edge retardance. Such an embedding series allows us to characterize the linearity of phase and principal retardance measured with uPTI. In the phase images of the beads, we see that phase values drop as the RI of the immersion oil approaches the RI of the bead. We see similar trend in the edge retardance signal from the principal retardance measurements. Plotting the theoretical phase and the measured phase versus the difference of the RI between beads and oils, we find the measured phase matches well with the theoretical one. We also see that edge retardance varies linearly with the difference of the refractive index between beads and oils, which is in agreement of the measurement from (48). However, in this case, no theoretical values are shown for edge retardance, because theoretical models for edge retardance are yet to be established.

\section{Spatial resolution}

Next, we characterize the spatial resolution of uPTI by imaging $300 \mathrm{~nm}$ polystyrene beads embedded in oil with RI of 1.5536 (fig. 2B). In the phase image, we can resolve individual beads. In the principal retardance image, we can resolve edge retardance of the beads in a form of small rings. To quantify the resolution, we select the center bead in the phase image for Gaussian fits in $x$ and $z$ directions. Deconvolving the physical size of the bead from the fitted Gaussians, we obtain the shape of the point spread functions (PSFs) in $x$ and $z$ directions. The full width at half maximum (FWHM) of this PSF in $x$ and $z$ directions show that we achieve a transverse FWHM of $230 \mathrm{~nm}$ and an axial FWHM of $810 \mathrm{~nm}$. We use FWHM of a theoretical image of a point (3) with a lens of 1.4 NA to benchmark the transverse and axial resolutions. The theoretical transverse FWHM is $190 \mathrm{~nm}(0.51 \lambda / \mathrm{NA})$ and the axial FWHM is $543 \mathrm{~nm}\left(2 \lambda / \mathrm{NA}^{2}\right)$. The theoretical axial resolution is a function of transverse spatial frequency of the specimen (26). For a spherical bead of finite spatial frequency, the FWHM can be worse than an infinitesimal point. Our measured transverse FWHM and axial FWHM compare well with the expected FWHM. These results also illustrate that our deconvolution algorithm and parameters do not introduce artifacts. As illustrated by images in fig. 3 and fig. 5, our measurements provide confocal-like 3D resolution that allows us to resolve cross-sections of single axons and bands of sarcomeres. 
bioRxiv preprint doi: https://doi.org/10.1101/2020.12.15.422951; this version posted December 16, 2020. The copyright holder for this preprint

(which was not certified by peer review) is the author/funder, who has granted bioRxiv a license to display the preprint in perpetuity. It is made available under aCC-BY-NC-ND 4.0 International license.

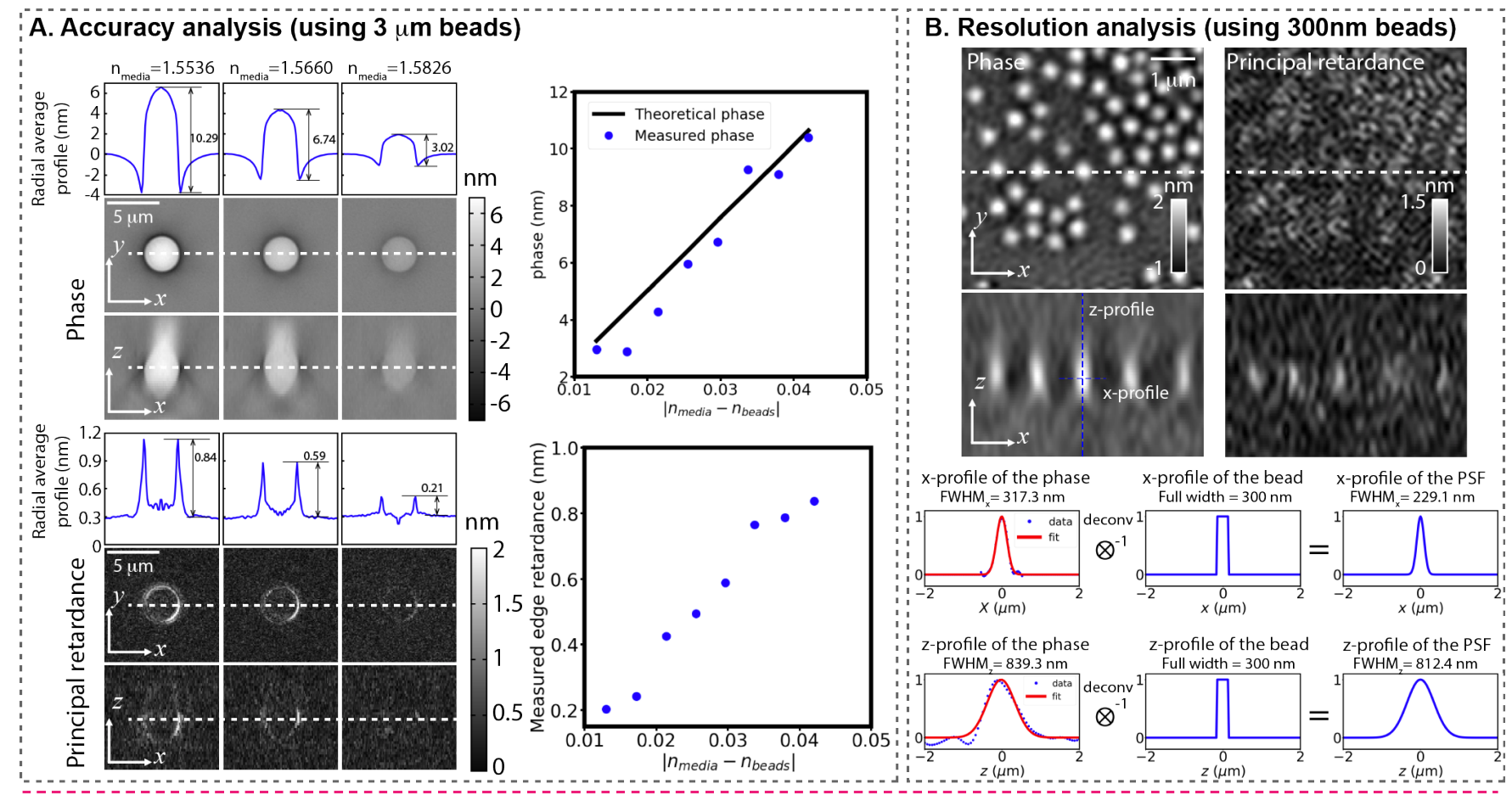

\section{Laser-written birefringent target}
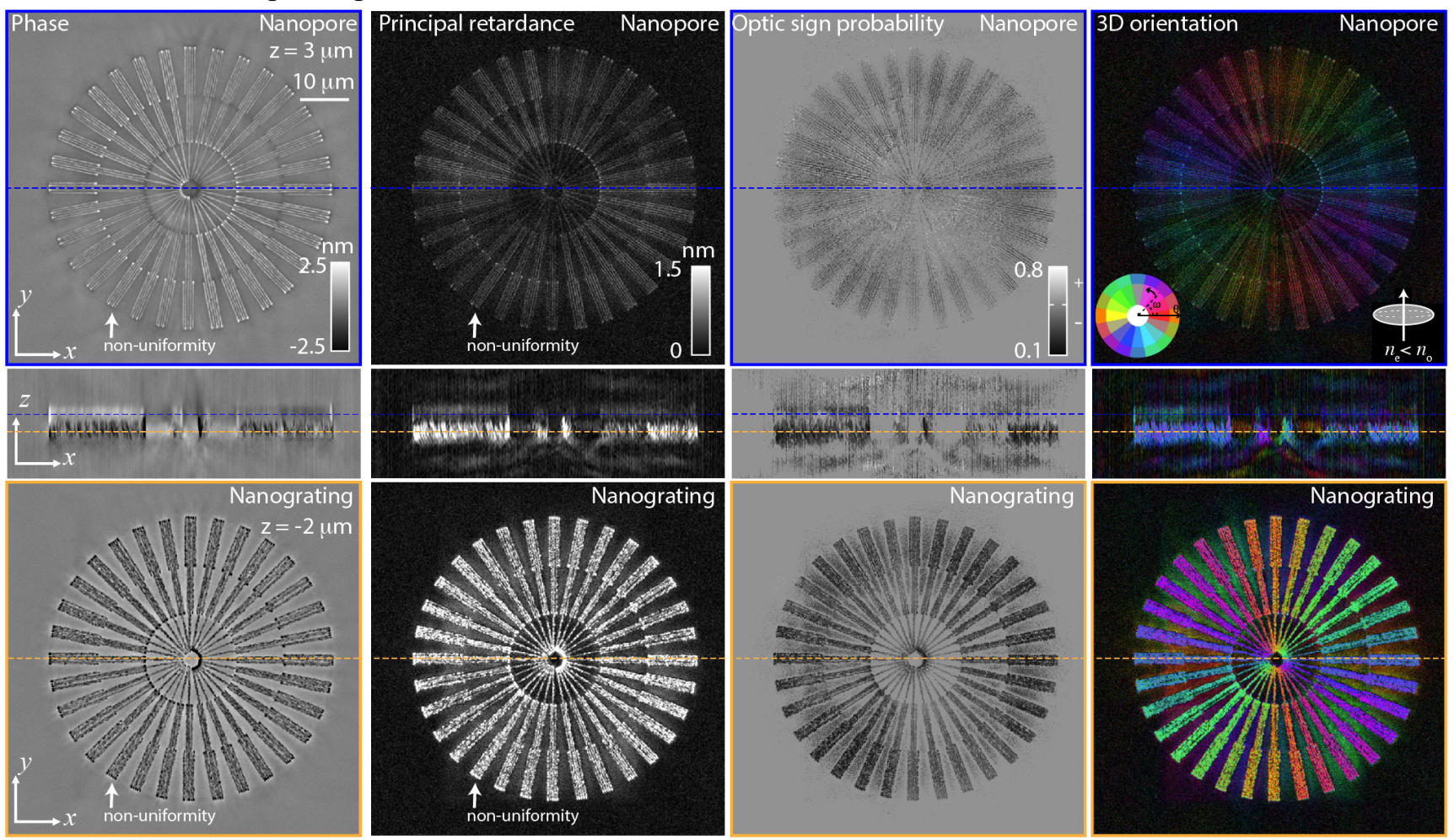

Fig. 2. Characterization of accuracy and resolution of uPTI: (A) Characterization of the accuracy of phase and principal retardance by imaging $3 \mu \mathrm{m}$ polystyrene beads immersed in oils with varying refractive indices (RI). Phase and principal retardance images of beads, and their corresponding radial average profiles show that measured phase and retardance decrease as the RI of the immersion oil approaches the RI of the bead. Plots of measured phase, theoretical phase, and edge retardance versus the difference of the RI of the immersion oil and the beads ( $n_{\text {beads }}=1.5956$ at $532 \mathrm{~nm}$ ) show that the measured values follow the expected trend (see Results). (B) Characterization of $3 \mathrm{D}$ spatial resolution by imaging $300 \mathrm{~nm}$ polystyrene beads immersed in oil with RI of 1.5536 . The phase images of beads show dense center and principal retardance images resolve edges of the beads. The phase of the center bead is selected for Gaussian fits in $x$ and $z$ directions. The Gaussian fits are deconvolved with the physical size of the bead to measure the full width at half maximum (FWHM) of the point spread function (PSF) in $x$ and $z$. (C) $x-y$ and $x-z$ slices of phase, principal retardance, optic sign probability, and $3 \mathrm{D}$ orientation volumes of a laser written glass enable determination of the nanograting and nanopore structures at different depths, for the first time to our knowledge. 


\section{Characterization of an anisotropic glass target}

Finally, we image a laser written anisotropic glass target shown in fig. 2C (through-focus video is shown in Video 1) to characterize the 3D orientation, verify estimation of optic sign, and demonstrate the utility of uPTI for metrology. The anisotropic target is made of fused silica modified with a polarized femtosecond laser (515 nm wavelength and 500-fs pulse duration) focused with 0.55 NA lens (Methods) (49). With uPTI, we identify two distinct laser-induced modifications (nanograting (50) and nanopore (19)) in different axial layers of the material. According to (19), nanograting modification of the material generates negative phase and stronger retardance, while nanopore modification generates positive phase and weaker retardance. uPTI estimates the target to have high probability of being negative uniaxial material, which agrees with the past observation (18, 19). The $x-y$ and $x-z$ sections through phase, principal retardance, and optic sign volumes match with these expected optical properties. Since this is a negative uniaxial material, the 3D orientation of the optic axis reports the fast axis of the material. We show 3D orientation in fig. 2C using the color sphere used in fig. 1D. The orientation of the optic axis in each spoke aligns with the $x-y$ plane and is orthogonal to the direction of the spokes, which matches with the axis of symmetry expected from the state of laser polarization used in the writing process. We clarify the same data with a RGB color sphere, which encodes 3D orientation by assigning RGB colors proportional to the projection of 3D orientation on $x, y$, and $z$ axes in Figure 2-supplement 1 . In addition, we measure subtle non-uniformity in phase and principal retardance at the ends of the line features along each spoke. These non-uniformities are due to aggregation of the modified glass, possibly associated with the trapping of defects within the focus of the scanning beam. Interestingly, the retardance and phase of the nanopore modification are stronger at the beginning of the structure, which indicates incubation mechanism, possibly associated with the trapping of defects during line scanning.

Another anisotropic target fabricated with different writing parameters (300-fs pulse duration, $515 \mathrm{~nm}$ wavelength, 0.55 NA) is shown in Figure 2-supplement 2 (through-focus video is shown in Video 2). This target has only one layer of nanograting modification. The double line-scan process used for writing this target eliminated the non-uniformities due to uneven aggregation of material. Collectively, these measurements show that uPTI can be a valuable technology for metrology and for reading novel optical storage devices, in addition to its use in biological imaging.

\section{C: Multi-scale imaging of mouse brain tissue with uPTI}

The architectural connectivity of mammalian brains can be inferred from the spatio-angular distribution of myelinated axons. MRI can provide measurement of spatio-angular distribution of axon bundles (44) and myelin fraction (52) with millimeter resolution. However, inference of the connectivity or pathology frequently requires micro-architectural ground truth (52, 53). Polarization microscopy is emerging as a label-free method for analyzing mesoscale connectivity and the architecture of brain tissue $(10-15,32,54)$ due to the following reasons: 1) High intrinsic anisotropy of the myelin sheath enables sensitive detection of distribution and orientation of axon fibers $(55,56), 2)$ Light microscopy can achieve sub-micron, single-axon resolution across large brains. Quantitative phase microscopy has also enabled imaging of brain architecture ex-vivo (57), in-vivo (58), and during disease (59).

A key limitation of all polarized light microscopy approaches has been that their light paths are not sensitive to the inclination of the 3D anisotropy and orientation. As a result, they report retardance and orientation projected on the microscope's image plane. Thus far, measurement of true anisotropy and 3D orientation of axons with diffraction-limited resolution has been an unsolved problem. With the diffraction-aware measurement of permittivity tensor, we reasoned that uPTI can measure the architecture of the brain tissue and enable high-resolution imaging of 3D orientation of axons. Here, we report measurements of phase, principal retardance, and 3D orientation at spatial scales ranging from $1 \mathrm{~cm}-1 \mu \mathrm{m}$ in $12 \mu \mathrm{m}$ thick sections of brain slices. At high resolutions, we acquire volumetric measurements and at low resolutions, we acquire planar measurements.

\section{D imaging of whole section}

First, we report planar (2D) measurements of phase, principal retardance, and 3D orientation of a section of adult mouse brain tissue. Figure 3A and 3B show the phase, principal retardance, and 3D orientation of an adult mouse brain located at level 51 of the Allen brain reference atlas (51). With the imaging and illumination NA of 0.55 (corresponding to spatial resolution of $\sim 0.5 \times 0.5 \times 3.2 \mu \mathrm{m}$ ), the imaging system measures anisotropy of myelin sheath averaged over whole axons. As a result, axons behave like a negative uniaxial material as we show in Figure 3-supplement 1B, whose 3D orientation is co-linear with axon axis $(55,56)$. Therefore, we assume all the axons are negative uniaxial material when computing 3D orientation at this resolution. Principal retardance and 3D orientation are rendered together with the brightness of the color encoding the principal retardance and color encoding the 3D orientation over the colorspehre. Phase shows overall morphology of the mouse brain, while principal retardance highlights the distribution of myelinated axons. As in other work (32, 54), important anatomical regions such as anterior commissure olfactory limb (aco), corpus callosum (cc), caudoputamen (CP), cortex (CTX), and ventricle (VL) are visible in both phase and principal retardance. In the 3D orientation image, we not only see the in-plane orientation aligned with the axon bundle, but also see a smooth transition in inclination from left to right over corpus callosum (yellow to pink colored region indicated by the top two white arrows in fig. 3B). We also notice that the left and right anterior commissure olfactory limb are inclined relative to the microscope axis (yellow and red colored stretches indicated by bottom two white arrows in fig. 3B). The same out-of-plane inclinations are also visible in bluish hue when the 3D orientation is 
bioRxiv preprint doi: https://doi.org/10.1101/2020.12.15.422951; this version posted December 16, 2020. The copyright holder for this preprint

(which was not certified by peer review) is the author/funder, who has granted bioRxiv a license to display the preprint in perpetuity. It is made available under aCC-BY-NC-ND 4.0 International license.
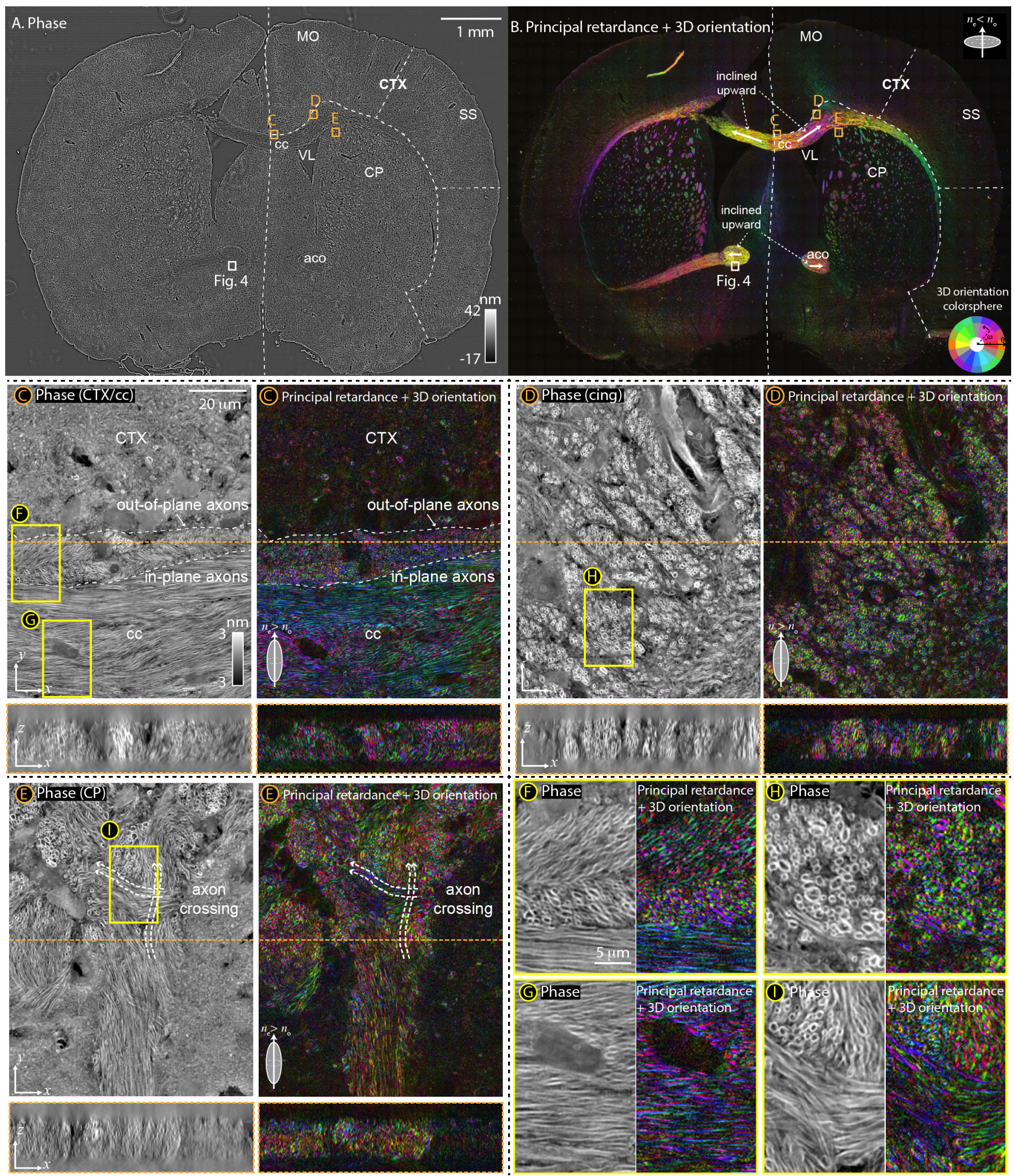

Fig. 3. Multi-scale imaging of the architecture of an adult mouse brain section with uPTI: (top row) $2 \mathrm{D}$ low-resolution imaging of the whole brain section with (A) phase, (B) principal retardance and 3D orientation channels. (bottom: C, D, and E) 3D high-resolution phase, principal retardance, and 3D orientation of three areas marked with orange boxes in the top row. At the imaging and illumination NA of 0.55 (spatial resolution of $\sim 0.5 \times 0.5 \times 3.2 \mu \mathrm{m}$ ), axons behave like negative uniaxial material (see fig. 4 . Therefore, we assume the negative uniaxial material when analyzing $3 \mathrm{D}$ orientation over the whole slide. Multiple anatomical landmarks are labeled according to the coronal section at level 51 of the Allen brain reference atlas (51), aco: anterior commissure (olfactory limb), cc: corpus callosum, CP: caudoputamen, CTX: cortex, MO: motor cortex, SS: somatosensory area, VL: ventricle. The white box is selected for further analysis in fig. 4. Following areas are imaged in high-resolution 3D mode with imaging NA of 1.47 and illumination NA of 1.4 (spatial resolution of $\sim 0.23 \times 0.23 \times 0.8 \mu \mathrm{m}$ ): (C) the boundary of the corpus callosum and the cortex region, (D) the cingulum bundle region, and $(E)$ the fiber crossing in the caudoputamen region. We further zoom in four parts from (C-E) to demonstrate complex axon networks in (F-I). With high resolution, we resolve the boundaries of individual axons, which behave as the positive uniaxial material with 3D orientation perpendicular to the surface of the membrane. 
encoded using RGB colorsphere Figure 3-supplement 1A.

\section{Volumetric, high resolution imaging of brain regions}

Next, we report high-resolution analysis of the brain tissue. Figure 3C-3E show $x-y$ and $x-z$ sections of three volumes imaged at high-resolution ( $1.47 \mathrm{NA}$, spatial resolution of $\sim 0.23 \times 0.23 \times 0.8 \mu \mathrm{m})$ in the section described above. Corresponding scans through $x-y, x-z, y-z$ sections are shown in Video 4-Video 6. At high resolution, uPTI measurements resolve myelin sheath around individual axons, which behaves like a positive uniaxial material with 3D orientation normal to the membrane (fig. 1A).

Figure 3C and Video 4 show the boundary between the cortex and the corpus callosum. While axons of corpus callosum are mostly parallel with the focal plane, axons at the boundary are inclined out-of-plane. The out-of-plane orientation of axons can be visually traced given high resolution of our data. Figure $3 \mathrm{~F}$ and $3 \mathrm{G}$ zoom in even further on the corpus callosum and on the boundary to highlight in-palne and out-of-plane patterns of axon organization. Figure 3D and Video 5 show rich out-of-plane axons distributed in the cingulum (cing) bundle region, which matches the result shown with correlative study of diffusion tensor imaging and fluorescence microscopy (60). Figure 3H zooms in further to show phase and 3D orientation of these out-of-plane axons. Figure 3E and Video 6 show the axon networks in the caudoputamen region, where we observe multiple axon crossings. A zoom in of a region with crossing axons is shown in fig. 3I. For all of the fields of view shown in fig. 3, the 3D orientation rendered with RGB color sphere and optic sign probability are shown in Figure 3-supplement 1. From 3D orientation images of fig. 3C-3I and Figure 3-supplement 1C-1I, we see that 3D orientation is radially oriented whether axons are traversing out-of-plane or in-plane, confirming that assumption of positive uniaxial anisotropy leads to reliable measurement of 3D orientation of myelin sheaths.

\section{Multi-resolution analysis}

Finally, we automate multi-scale imaging to acquire uPT measurements of mm-sized tissue sections with sub-micrometer 3D resolution. We automate this tiled acquisition using Micro-Manager (https://github.com/micro-manager), a python bridge to Micro-Manager (https://github.com/czbiohub/mm2python), and GPU-accelerated computational pipeline implemented on a compute cluster (Methods). We designed the analysis pipeline to enable robust reconstruction of uPT at any scale spanned by the acquisition. Measurements at larger scales are computed by filtering the components of the scattering potential tensor, which are then transformed into phase, retardance, and 3D orientation. Results of one such multi-scale analysis of the right corpus callosum region are shown in Video 3. At spatial scales larger than the typical size of axons, we compute the 3D orientation assuming a negative uniaxial material. When axon cross-sections are resolved, we visualize complex axon networks by displaying the phase and principal retardance through focus and at multiple locations. The data suggests that 3D orientation of axons detected at low resolution follows the same trend with the 3D structural orientation of axons at high-resolution.

We verify the quantitative correspondence between $3 \mathrm{D}$ orientation distributions measured with low-resolution (20x, $0.55 \mathrm{NA})$ and high-resolution $(63 \mathrm{x}, 1.47 \mathrm{NA})$ acquisitions. We image the $3 \mathrm{D}$ orientation in the white box in fig. $3 \mathrm{~B}$ at highresolution. We low-pass filter the high-resolution data to have similar spatial resolution as the low-resolution data and compute the 3D orientation histogram of within two sub-regions as shown in Figure 3-supplement 2. The azimuthal dimension of this histogram shows the in-plane orientation, $\omega$, and the radial dimension shows the inclination relative to the imaging axis, $\theta$. The histograms of 3D orientation of axon bundles in the low-resolution data and smoothed 3D orientation computed from highresolution data agree well, confirming that our pipeline provides physically meaningful measurements across spatial scales. These results also indicate that uPTI provides sensitive measurement of 3D anisotropy below the spatial resolution limit of the microscope, and uPT measurements with $\sim 3 \mu \mathrm{m}$ resolution can be used for fast imaging of 3D orientation of axons.

\section{D: Missing cone of orientations}

The sensitivity and resolution of spatio-angular imaging methods, such as DTI and uPTI, depend on the spatial frequency and 3D orientation of the structures relative to the geometry of the imaging system. Given the circularly symmetric, single axis design of uPTI, its spatial and angular resolution is uniform in $x-y$ plane. The range of orientations accessible by a microscope depend on the strength of the electric field components along the $x, y, z$-axes. In other words, the range of uPT inclinations that can be probed by a microscope is fundamentally limited by the highest angle of the plane wave that can traverse through the light path. Our 1.4NA illumination module provides state-of-the-art resolution for inclination relative to other methods $(23,24,32)$ that provide no resolution for inclination. Nevertheless, the orientations with the smallest inclination near $z$-axis remain inaccessible. In other words, just as the single view imaging systems exhibit the missing cone of spatial frequencies along the imaging axis, they also exhibit the 'missing cone of orientations' around the imaging axis. The analogous effect is also observed during spatio-angular imaging of fluorescent dipoles $(41,61,62)$. Detailed analysis of the missing cone of orientations in transmission imaging is beyond the scope of this paper. Here, we characterize the missing cone of orientations in the current design by evaluating the uPT measurements of axons as the inclination of axons changes.

High-resolution 3D orientation images of the axon networks in fig. 3C-3I show radial arrangement around the axis of axons suggesting that the anisotropy arises from the myelin sheath. If these 3D orientation measurements are accurate, the angular distribution of 3D orientation would follow the orientation of lipids, i.e., orientations perpendicular to the axon as the inclination 
bioRxiv preprint doi: https://doi.org/10.1101/2020.12.15.422951; this version posted December 16, 2020. The copyright holder for this preprint

(which was not certified by peer review) is the author/funder, who has granted bioRxiv a license to display the preprint in perpetuity. It is made available under aCC-BY-NC-ND 4.0 International license.
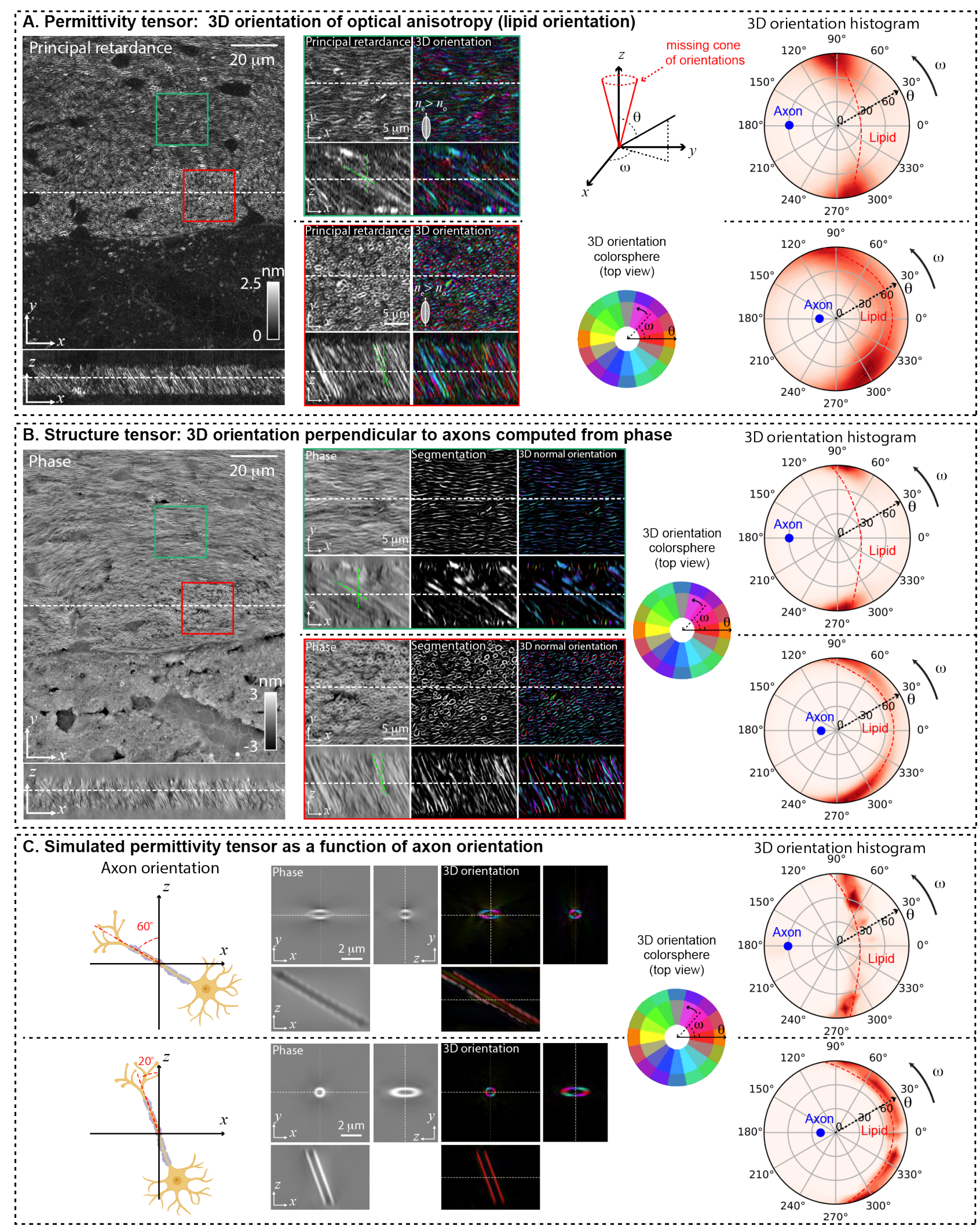

Fig. 4. Evaluation of the missing cone of orientations in uPT measurements with structure tensor analysis and wave optical simulations: Volumes of mouse brain architecture in anterior commissure region highlighted by the white box in fig. 3 shows a variety of 3D axon orientations. We compare 3D orientation distribution of the measured permittivity tensor (A), computed structure tensor (B), and simulated permittivity tensor (C). (A) (left) $x$-y and $x-z$ sections through principal retardance volume, (right) Principal retardance and $3 \mathrm{D}$ orientation of two volumes identified with green (axons tilted $60^{\circ}$ to the left from $z$-axis) and red (axons tilted $20^{\circ}$ to the left from $z$-axis) boxes. The histograms show the distribution of the 3D orientation in the two cropped volumes, indicating 3D orientation of the lipids (red) around axons. To aid interpretation, we placed a blue dot in each histogram to indicate the corresponding axon orientation in the selected volume. (B) (left) $x-y$ and $x-z$ sections through phase volume, (right) Phase, axon segmentation maps, and 3D orientation from the structure tensor of two corresponding volumes. The histograms show the distribution of the 3D orientation that is normal to the axon cross section. (C) We simulate a single axon oriented the same as the experiment to model expected phase, principal retardance, and 3D orientation. The histograms of the simulated 3D orientation shows distribution consistent with the measured permittivity tensor and the computed structure tensor. 
of axons changes. Figure 4 shows the distribution of 3D orientation evaluated by three different approaches as inclination of axons changes: 1) measured 3D orientation of the permittivity tensor , 2) computed 3D orientation based on the structure tensor analysis of the phase, and 3) simulated 3D orientation of a single axon modeled as a positive uniaxial cylindrical shell. With this analysis, we validate the range of validity of the 3D orientation measurements. Interestingly, we find that the missing cone of orientations affects accuracy of not only principal retardance, orientation, and optic axes (measures of anisotropy and symmetry), but also the accuracy of phase.

\section{Missing cone of orientations in measured permittivity tensor}

Figure 4A shows the principal retardance and 3D orientation of the uPT in the anterior commissure olfactory limb region cropped from the white box in fig. 3A and 3B. Two volumes with different axon inclinations are chosen (green and red regions of interest, ROIs) for further analysis. From the $x-z$ sections of these two volumes, we recognize that the green box contains axons mostly inclined at $60^{\circ}$ to the left of the $z$-axis and the red box contains axons mostly inclined at $20^{\circ}$ to the left of the $z$-axis. To visualize the orientation distribution of the lipids, we plot the histogram of the 3D orientation inside these two volumes. If the inclination of the axon is $0^{\circ}$ from the $z$-axis, we expect orientation of lipids to be in the focal plane evenly distributed in the azimuthal dimension, which will be a distribution around a circle with radius $\theta=90^{\circ}$ (The top row of Figure 4-supplement 3). With $20^{\circ}$ and $60^{\circ}$ inclinations of axons, we see a gradual rotation of this circle (collective 3D orientation of lipids) to the left side of the histogram, as expected. At high inclinations of the axon, we notice a gradual reduction in the density of orientations of lipids as lipids align along the $z$-axis. This drop in sensitivity is due to the poor transfer of optical properties of anisotropic material to intensity modulations as the material aligns with $z$-axis, i.e., the missing cone of orientations.

\section{Missing cone of orientations in computed structure tensor}

We evaluate the accuracy of phase measurements by analyzing the 3D orientation of lipids using a structure tensor computed from phase (Methods) of the same region as shown in fig. 4B. Phase is a measure of the isotropic component of uPT, independent from the 3D anisotropy (principal retardance and 3D orientation). The resolved axon structure in the phase image provides an independent source of 3D orientation measurement. The largest eigenvector of the structure tensor of the phase image reports the 3D orientation along the lipids and the smallest eigenvector reports the 3D orientation of the axis of axon. We show phase, segmentation map computed from eigenvalues of structure tensor, and 3D orientation of the largest eigenvector, which corresponds to the optically measured 3D orientation shown in fig. 4A. The 3D orientation from the phase image is also radially directed around axons like in the previous case. The histograms of this $3 \mathrm{D}$ orientation shows a good agreement with the histogram in fig. 4A. We again observe the reduction in the density of orientations of lipids when lipid orientation from structure tensor is close to the imaging axis.

In addition to verification of the lipid orientation, we also compare the axon orientation measured from the structure tensor (Figure 4-supplement 2 with axon orientation measured from the permittivity tensor (Figure 3 -supplement 2). We find that when the uPT orientation is outside the missing cone $\left(\theta \ll 0^{\circ}\right)$, both estimates of 3D orientation agree, but when the uPT orientation is inside the missing cone $\left(\theta \sim 0^{\circ}\right)$, both estimates disagree.

\section{Verification with simulations}

In order to better understand the boundaries of the missing cone, we implement simplified simulation of the imaging of single axons oriented at multiple inclinations. Figure $4 \mathrm{C}$ shows results of simulation for axons inclined $60^{\circ}$ and $20^{\circ}$ to the left from the $z$-axis. In this simulation, we assume an axon is a perfect cylindrical shell composed of uniform positive uniaxial material with $3 \mathrm{D}$ orientation pointing radially from the center of the cylinder. In the reconstruction of the simulations, we see that the circular cross-section of the axon is more visible in both the phase image and the 3D orientation image when the axon is inclined at $20^{\circ}$ than inclined at $60^{\circ}$, confirming our experimental observations. The phase depends on the inclination, because the estimate of the isotropic component is affected by the anisotropic component as shown in Eq. (12). The principal retardance becomes noisy as $\theta$ approaches $0^{\circ}$ as shown in Eq. (13). This effect also leads to a drop of the density in the 3D orientation histogram as the orientation of lipids approaches the imaging axis (when the axon is inclined at $60^{\circ}$ ). Comparison of the $3 \mathrm{D}$ orientation in the simulation (fig. 4C) with the 3D orientation in the experiment (fig. 4A) and the structure tensor analysis (fig. 4B) provides a calibration of the uPTI measurements as a function of inclination.

We also simulated single axons inclined at a range of angles as shown in Figure 4-supplement 3. The histogram of the 3D orientation shows a distribution that gradually shifts to the left when the axon is inclined more towards the focal plane, which is consistent with our observation. Through the simulation, we also found that the optic sign tends to be predicted as positive when the axon is aligned along the imaging axis (i.e. the lipids are oriented in-plane). When axons are inclined more in-plane (i.e. the lipids are oriented out-of-plane), the optic sign probability becomes noisier due to lack of sensitivity as $\theta$ approaches $0^{\circ}$ (the missing cone of orientations). The optic sign prediction also leans towards negative uniaxial. We observe similar trend in optic sign measurements as shown in Figure 4-supplement 1. This transition from positive to negative uniaxial optic sign could be because of the missing cone of orientations $\left(\theta \sim 0^{\circ}\right)$ or the averaging of the permittivity tensor within the spatial resolution of the microscope. The single-view implementation of uPTI we report provides higher resolution along the $x, y$ axes as compared to $z$ axis and hence the measurement is averaged over longer spatial extent along $z$ axis. When the axon is inclined in-plane, 
bioRxiv preprint doi: https://doi.org/10.1101/2020.12.15.422951; this version posted December 16, 2020. The copyright holder for this preprint (which was not certified by peer review) is the author/funder, who has granted bioRxiv a license to display the preprint in perpetuity. It is made available under aCC-BY-NC-ND 4.0 International license.

a microscope may average the axon membrane that covers wider distribution of the orientation than when the axon is inclined out-of-plane, causing the surrounding voxels to appear as negative uniaxial material.

Collectively, above data and analyses begin to illustrate the effect of missing cone of orientations in label-free spatio-angular imaging. Above analyses characterizes the range of validity of current implementation of uPTI and offers directions for further improvements in uPT measurements.

\section{E: Multiplexing of uPTI and fluorescence for imaging cardiomyocytes}

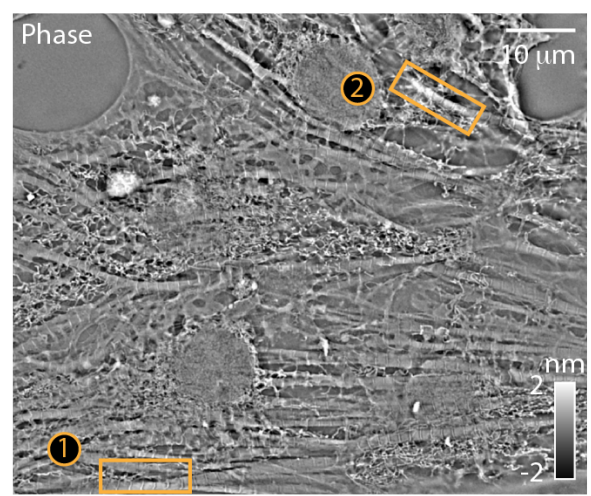

B. Schematic of sarcomere architecture

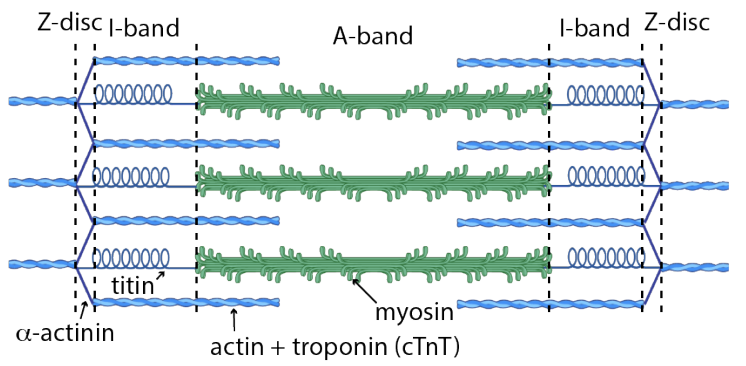

\section{A. Multi-modal imaging of cardiomyocytes}
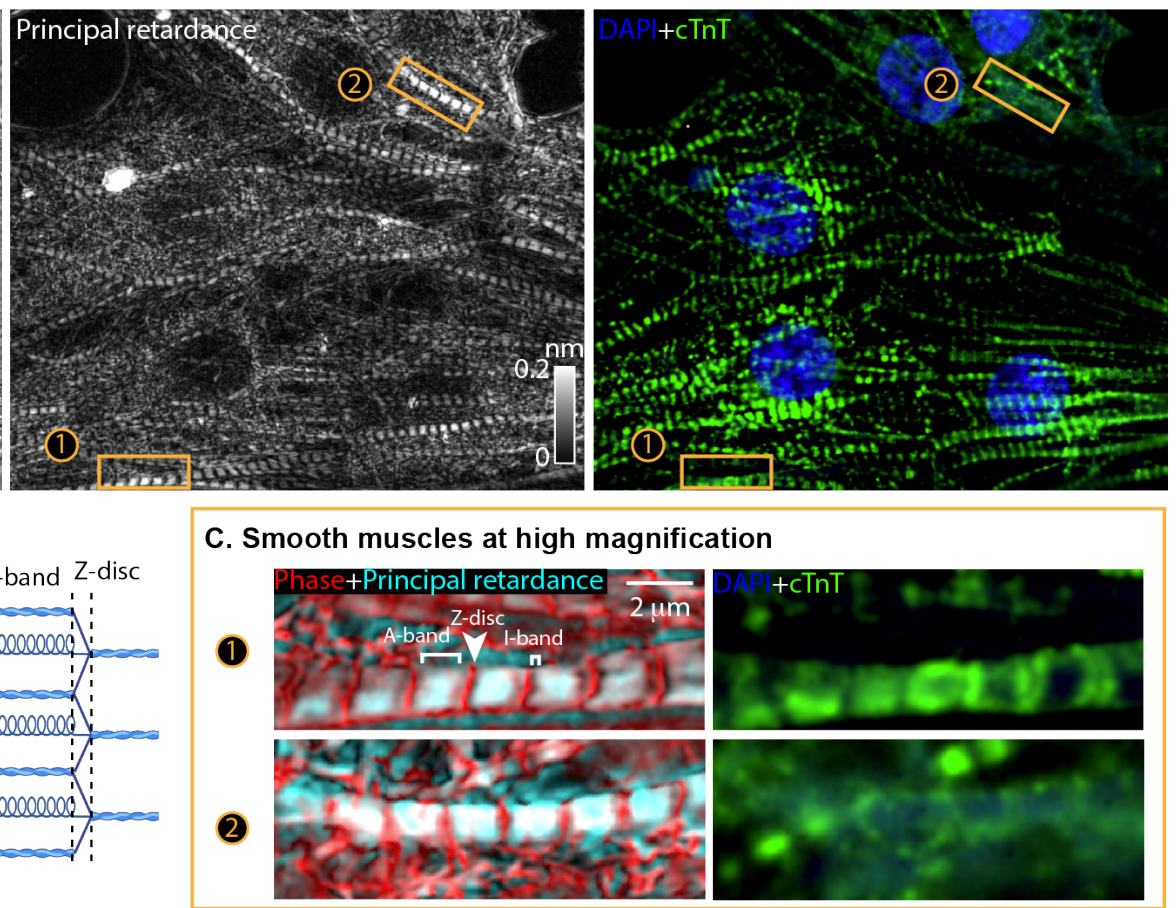

Fig. 5. Imaging physical and molecular architecture of fixed iPSC-derived cardiomyocytes: (A) Phase, principal retardance, and fluorescence images (overlay shows DAPI stain in blue, cTnT stain in green, and dsRNA stain in red) of the healthy iPSC-derived cardiomyocytes. (B) A schematic of the sarcomere architecture shows its key molecular components and their organization to enable interpretation of the images. (C) Two zoomed regions of the smooth muscle are shown with label-free channel (overlay of phase in red and principal retardance in cyan) and the fluorescence channel. Z-disc, A-band, and I-band can be identified in the label-free overlay. A-band and I-band are visible from variations in phase and principal retardance, and Z-disc is visible due to high phase and low retardance. In zoom (1), the cTnT label shows troponin in the actin-rich regions of the sarcomere, overlapping with both I-band and A-band. The zoom (2) shows weak fluorescence signal due to labeling stochasticity, but the sarcomeric architecture is visible in the label-free imaging.

Quantitative label-free imaging is emerging as a valuable method for discovery of biological mechanisms in disease-relevant cell and tissue models, because it provides unbiased and consistent readouts of physical architecture of diverse cell types. On the other hand, immunolabeling reports the molecular architecture. We designed and implemented uPTI to enable multiplexing with a wide-field fluorescence deconvolution imaging, which enables mapping of both physical and molecular architecture of cells at confocal-like spatial resolution. We employ this approach to analyze organelle architecture of cardiac muscle cells (cardiomyocytes).

Induced pluripotent stem cell (iPSC)-derived cardiomyocytes (CM) have emerged as a genetically editable model to study cardiac diseases (63) and drug screening (64). CM are highly specialized contractile cells, so studying the architecture of the myofibril and its building blocks, the sarcomeres, is of critical importance to characterize their function (63). Current methods rely on fluorescent imaging of fixed cells, which can only be done as a terminal assay, or cell lines expressing a fluorescent reporter, which limits its application to new specimens (for example cells derived from different patients). In addition, reliance on a single protein as a reporter for sarcomere structure limits the information that can be obtained.

Figure 5A and the corresponding through-focus video Video 7 show images of fixed iPSC-derived CMs with the labelfree channels (phase and principal retardance) and the fluorescence channels. Polarized light imaging has played an important role in understanding architecture and activity of sarcomeres (65) (fig. 5B). In fact, the A- and I-bands of sarcomeres were named after the anisotropic and isotropic bands first observed in muscle tissue with polarized light microscopy. The phase image shows nuclei, myofibrils, and a crowded meshwork of membranous organelles surrounding sarcomeres. The principal retardance shows the distinct striated pattern of myofibrils. The CMs are stained with DAPI (blue) to label DNA and fluorescent antibody (green) against thin filament marker cardiac Troponin $\mathrm{T}(\mathrm{cTnT})$ to label sarcomeres. We see agreement between the label-free channels and the fluorescence channels in terms of the locations of the nuclei and the sarcomere, however retardance image shows sarcomere organization more consistently than cTnT labeling. 
The schematic in fig. 5B shows sarcomere architecture and its key molecular components to enable interpretation of the images. Each sarcomeric unit is bracketed by two Z-discs, composed of densely packed proteins including $\alpha$-actinin. Between Z-discs, the sarcomere is organized in an I-band (isotropic band) and an A-band (anisotropic band). An I-band is mainly composed of thin actin filaments, while an A-band contains thick myosin filament. We zoom in on two regions of fig. 5A to examine sarcomeric structures in fig. 5C. We display the label-free channels of these two ROIs with an overlay of phase in red and principal retardance in cyan, from which we can clearly resolve sarcomeric components. The phase channel emphasizes the electron-dense Z-disc region. In between Z-discs, we see both strong phase and principal retardance that come from the anisotropic thick myosin filaments, which defines the A-band. We further notice spacing between Z-discs and A-bands. This spacing has lower phase and almost no principal retardance, i.e., it is less dense and isotropic. Comparing its location and size with the transmission electron microscopy images of CMs (66), we identify it as the I-band structure. Figure 5C also shows corresponding fluorescence images for the same ROIs. Since the cTnT is localized in both I-band and A-band, we see most of the signal between two Z-discs in ROI (1) of the fluorescence image. Troponin label in ROI (2) of the fluorescence image shows almost no signal for the sarcomere, while there is a strong signal of sarcomeres detected by the label-free channels. This suggests missing label due to inability of the antibody to bind cTnT or mislocalization of cTnT. Here, label-free imaging complements the potential instability of staining by providing consistent physical measurements of sarcomeres without relying on the labeling of a specific factor. Figure 5-supplement 1 shows the 3D orientation and optic sign probability of the corresponding field of view. The 3D orientation aligns well along with the orientation of myofibrils and the optic sign probability suggests that thick filaments in the sarcomere behave as a positive uniaxial material.

The ability to resolve Z-discs and small I-bands further illustrate high resolution and sensitivity of uPT, and presents it as a feature-rich tool to evaluate sarcomeric structure and maturity. These results show that complementary information can be gained in the architecture of cardiac cells and tissues using uPTI multiplexed with fluorescence imaging. These results present uPTI as a powerful tool for the modeling of sarcomeric cardiomyopathies (63), for screening of cardiotoxic or cardioprotective drugs (64), or for the development of methods to improve iPS-CM maturity (67). It also can be applied to other valuable muscle specimens that are challenging to label, such as primary cardiac or skeletal myocytes, or for the non-disruptive imaging of sarcomeric architecture in live muscle cells without the need of engineering fluorescent reporter cell lines. Furthermore, the same approach can be extended to analysis of changes in the organelle architecture during disease-relevant perturbations, such as infection of CMs by SARS-CoV-2 virus (66), which opens new opportunities for image-based rich phenotyping of the cellular architecture.

\section{Discussion}

We have demonstrated that uPTI provides a more complete description of a specimens' uniaxial permittivity tensor compared to previously reported quantitative phase and polarization microscopy. We have shown that it can be applied to analysis of the architecture of a laser-written anisotropic glass, a mouse brain tissue section, and iPSC derived cardiomyocytes. We also illustrated how the uniaxial permittivity tensor can be interpreted in terms of the physical properties of the specimen and as a function of its 3D orientation. We have implemented automated acquisition and analysis that enable multi-resolution analysis of tissue architecture. Next, we describe how we chose to balance the trade-offs among spatial resolution, temporal resolution, sensitivity, and complexity when designing and implementing uPTI. We also discuss future directions of research enabled by this work.

We have designed uPTI for easy adoption by leveraging commercially available modules and by providing an open-source algorithm. uPTI can be implemented on a commercial microscope by adding an LCD panel, a circular polarizer, and a machine vision polarization camera. This design minimizes physical movement of the specimen during the data acquisition, except for $z$-scanning when imaging 3D specimens and $x y$ scanning when tiling a large specimen. In the present implementation, uPTI is too slow to enable live imaging because of slow refresh rate and slow software communication of the Adafruit LCD. However, faster electronic control of phase and polarization diversity and a more advanced LCD panel (e.g., transmissive spatial light modulator) will enable rapid improvements in the acquisition speeds.

Relative to the existing label-free microscopy methods that report both density and anisotropy (23-25), uPTI is simpler and amenable to high speed acquisition. Despite the relative simplicity of hardware, uPTI provides more complete and quantitative measurements as compared to the methods described in (23-25, 32). uPTI employs partially coherent illumination and propagation-related optical effects to transform invisible density and anisotropy into visible intensities. This optical design makes it easy to multiplex uPTI with other imaging modalities, such as fluorescence (as we demonstrated), H\&E staining, and spatial transcriptomics. The partially coherent illumination can provide $2 \times$ resolution as compared to approaches that use coherent illumination. Synthetic aperture imaging with coherent illumination (24) can approach the same resolution, but has not yet been developed to measure 3D anisotropy. The partial coherence also makes the measurements robust to speckle noise that commonly affects label-free imaging methods (68). Relative to the interference-based optical designs $(20,69,70)$, our non-interferometric design achieves better robsutness to speckle noise by trading-off the sensitivity to low spatial frequencies in phase measurements, which correspond to large-scale variations in the specimen $(27,34)$. uPTI detects polarization-sensitive modulations with a compact polarization camera through linear polarizers embedded on top of the sensor. Our current choice 
of using a machine vision polarization camera guarantees simple setup and robust calibration, but it is not as sensitive to small changes in retardance as the polarization imaging based on elliptical states $(30,32)$. We are currently extending the design of uPTI to utilize elliptical states, which should lead to higher sensitivity to small changes in anisotropy of biological or fabricated specimens.

Volumetric analysis of the architecture of the mouse brain tissue and cardiomyocytes illustrate that a high-NA variant of uPTI can measure density and 3D anisotropy with confocal-like resolution, for the first time to our knowledge. The physical architecture accessible with uPTI is complementary to the molecular architecture that can be imaged with multiplexed fluorescence. The sensitivity and resolution of our data indicate that the measurements provided by uPTI can enable new studies in demyelinating diseases, changes in organelle architectures of infected cells, mechanobiology, among other fields. Improvements to sensitivity and speed discussed above will widen the range of biological questions that can be addressed with uPTI. We have employed uPTI to characterize laser-written anisotropic glass, which is a rapidly emerging high density optical storage technology. Our measurements provided one of the first 3D volumes of phase, retardance, and 3D orientation, which have required distinct instruments before. UPTI can therefore provide a foundation for developing readers of such optical storage devices, which further motivates the improvements in speed and sensitivity.

Multimodal label-free measurements provided by UPTI is its key advantage, but these channels of information experience varying amounts of noise. In particular, the estimation of the optic sign and the inclination of the 3D orientation become unstable when the optic axis is aligned with the imaging axis. This occurs, because imaging with a single lens cannot probe the specimen with polarization states that are aligned with the imaging axis. Nevertheless, we show that the high-NA illumination version of uPTI provides adequate sensitivity to map inclination of axons in mouse brain tissue and enables estimation of optic sign in 2D or 3D space (fig. 4). As far as we know, the estimation of optic sign in 2D or 3D space has never been possible before. Further improvements in robustness of inclination and optic sign can be achieved using ellipitical polarization states that reduce noise or using tilted sensors that enable probing of the axial electric field components.

The deconvolution algorithm of uPTI is designed to reconstruct linear contribution of the uPT to the recorded intensities under the assumption of single-scattering. The assumptions of single-scattering and linearity are applicable to many thin specimens that are mostly transparent. These assumptions also simplified the estimation of components of the scattering potential tensor to a one-step Fourier space division using Cramer's rule. The multi-channel deconvolution is implemented with multiple regularizers to account for different amounts of noise observed in different tensor components. These regularizers are hyper parameters that could introduce biases in the reconstruction unless tuned by a knowledgeable user. These biases can be eliminated by reducing the noise level in the measurement or by identifying a systematic relationship among regularization parameters or by developing priors that can be incorporated in the iterative implementation of the deconvolution algorithm.

Spatio-angular measurements akin to uPTI are being developed with fluorescence polarization imaging $(41,61,62)$. The fluorescence imaging is described by incoherent image formation, which is more amenable to linear approximation. We anticipate that the models and deconvolution approaches we have reported will be of value to spatio-angular fluorescence imaging as well. Similarly, modeling and deconvolution of permittivity tensor can be further refined by adopting models and deconvolution algorithms developed for spatio-angular fluorescence imaging and DTI.

Taken together, we report the unique capability of measuring the uniaxial permittivity tensor of diverse specimens using simple add-on modules on a commercial microscope and open-source diffraction-aware deconvolution algorithm. uPTI has already allowed us to image 3D axon bundle orientation across a mouse brain slice, single axons within complex networks, and the organelle architecture of cardiomyocytes. The comprehensive analysis of architecture enabled by uPTI can provide insights in open questions of fundamental importance and lead to markers of clinical relevance in the biology community. Similarly, it can enable quantitative analysis and discovery of new material properties in the material science community.

\section{Methods}

\section{A: Forward model}

In order to deconvolve components of the uniaxial permittivity tensor (uPT) from the intensity measurement, we first develop a forward model that relates the uPT to measured intensities. Our model integrates vector wave equation $(71,72)$ and partially coherent scalar imaging model (73-75) assuming Born approximation (3), i.e., single-scattering. This partially coherent, vectorial model linearly links the measured Stokes parameters with the 3D distribution of the permittivity tensor of a weakly scattering specimen through transfer functions that describe the optical path. The model is summarized here and the detailed derivation is provided in Supplementary notes 1.

\section{A.1: Permittivity and scattering potential tensor}

The permittivity tensor of an uniaxial material oriented with in-plane orientation of $\omega$ and inclination of $\theta$ as shown in fig. 1B is expressed as

$$
\overline{\bar{\epsilon}}_{\mathrm{r}}=\left[\begin{array}{ccc}
\epsilon_{\mathrm{r}}-\Delta \epsilon_{\mathrm{r}}\left(\cos ^{2} \theta-\sin ^{2} \theta \cos 2 \omega\right) & \Delta \epsilon_{\mathrm{r}} \sin ^{2} \theta \sin 2 \omega & \Delta \epsilon_{\mathrm{r}} \sin 2 \theta \cos \omega \\
\Delta \epsilon_{\mathrm{r}} \sin ^{2} \theta \sin 2 \omega & \epsilon_{\mathrm{r}}-\Delta \epsilon_{\mathrm{r}}\left(\cos ^{2} \theta+\sin ^{2} \theta \cos 2 \omega\right) & \Delta \epsilon_{\mathrm{r}} \sin 2 \theta \sin \omega \\
\Delta \epsilon_{\mathrm{r}} \sin 2 \theta \cos \omega & \Delta \epsilon_{\mathrm{r}} \sin 2 \theta \sin \omega & \epsilon_{\mathrm{r}}+\Delta \epsilon_{\mathrm{r}} \cos 2 \theta
\end{array}\right],
$$


where

$$
\begin{aligned}
\epsilon_{\mathrm{r}} & =\frac{1}{2}\left(n_{\mathrm{e}}^{2}+n_{\mathrm{o}}^{2}\right) \\
\Delta \epsilon_{\mathrm{r}} & =\frac{1}{2}\left(n_{\mathrm{e}}^{2}-n_{\mathrm{o}}^{2}\right),
\end{aligned}
$$

$n_{\mathrm{o}}$ and $n_{\mathrm{e}}$ are refractive indices (RI) experienced by the ordinary and extraordinary wave, respectively. Diffraction tomography approaches have relied on the scattering potential (45) and scattering matrix (46) models to reconstruct volumetric distribution of density and projected anisotropy, respectively. We extend this concept and model scattering potential tensor to reconstruct volumetric distribution of density, 3D anisotropy, and material symmetry. The scattering potential tensor is defined as,

$$
\begin{gathered}
\overline{\bar{f}}=k_{0}^{2}\left(\overline{\bar{\epsilon}}_{\mathrm{r}}-\epsilon_{\mathrm{rm}}\right)=\left[\begin{array}{ccc}
f_{0}+f_{1 \mathrm{c}} & f_{1 \mathrm{~s}} & f_{2 \mathrm{c}} \\
f_{1 \mathrm{~s}} & f_{0}-f_{1 \mathrm{c}} & f_{2 \mathrm{~s}} \\
f_{2 \mathrm{c}} & f_{2 \mathrm{~s}} & f_{0}+f_{3}
\end{array}\right], \\
\text { where } \quad\left\{\begin{array}{l}
f_{0}=k_{0}^{2}\left(\epsilon_{\mathrm{r}}-\epsilon_{\mathrm{rm}}-\Delta \epsilon_{\mathrm{r}} \cos ^{2} \theta\right) \\
f_{1 \mathrm{c}}=k_{0}^{2} \Delta \epsilon_{\mathrm{r}} \sin ^{2} \theta \cos 2 \omega \\
f_{1 \mathrm{~s}}=k_{0}^{2} \Delta \epsilon_{\mathrm{r}} \sin ^{2} \theta \sin 2 \omega \\
f_{2 \mathrm{c}}=k_{0}^{2} \Delta \epsilon_{\mathrm{r}} \sin 2 \theta \cos \omega \\
f_{2 \mathrm{~s}}=k_{0}^{2} \Delta \epsilon_{\mathrm{r}} \sin 2 \theta \sin \omega \\
f_{3}=k_{0}^{2} \Delta \epsilon_{\mathrm{r}}\left(3 \cos ^{2} \theta-1\right)
\end{array}\right.
\end{gathered}
$$

$k_{0}=2 \pi / \lambda_{0}$ is the free-space wavenumber, $\lambda_{0}$ is the free-space wavelength of the light, and $\epsilon_{\mathrm{rm}}$ is the isotropic relative permittivity of the surrounding media. This scattering potential tensor contains the same information as the permittivity tensor of the specimen, except that the scattering potential tensor loses the information of the relative permittivity of the surrounding medium. Subsequently, we will focus on developing a mapping between the scattering potential tensor and the Stokes parameters for our deconvolution algorithm.

\section{A.2: Scattered electric field with Born approximation}

The Stokes parameters are measurements of the scattered electric field. The scattered electric field comes from the interaction between the incident light and the scattering potential tensor, and it is derived based on the vector wave equation $(71,72)$ and Born approximation (3) as

$$
\vec{E}_{\mathrm{out}}(\vec{r}) \approx \vec{E}_{\mathrm{inc}}(\vec{r})+\iiint \overline{\bar{G}}\left(\vec{r}-\vec{r}^{\prime}\right) \overline{\bar{f}}\left(\vec{r}^{\prime}\right) \vec{E}_{\mathrm{inc}}\left(\vec{r}^{\prime}\right) d^{3} \vec{r}
$$

where $\vec{E}_{\text {out }}(\vec{r})=\left[E_{\text {out }, x}(\vec{r}), E_{\text {out }, y}(\vec{r}), E_{\text {out }, z}(\vec{r})\right]^{T}$ is the scattered output electric field in 3D space $\vec{r}=[x, y, z]^{T}, \vec{E}_{\text {inc }}(\vec{r})$ is the incident electric field, and $\overline{\bar{G}}(\vec{r})$ is the dyadic Green's tensor. This equation describes a single scattering event from a coherent incident wave. In our experiment, we adopt partially coherent illumination, which brings a summation of multiple coherent scattering events to the detection. We will further discuss this effect in the Stokes representation.

\section{A.3: Stokes parameters under partially coherent illumination}

We first define the scattered electric field with incident light at spatial frequency of $\vec{\nu}_{\perp}$ to be $\vec{E}_{\text {out }}\left(\vec{r}, \vec{\nu}_{\perp}\right)$. Then, the Stokes parameters under the $\alpha$-th partially coherent source pattern are sums of the contribution from individual coherent scattering events and are expressed as in $(73,74)$

$$
\begin{array}{ll} 
& {\left[\begin{array}{c}
S_{0, \alpha}(\vec{r}) \\
S_{1, \alpha}(\vec{r}) \\
S_{2, \alpha}(\vec{r}) \\
S_{3, \alpha}(\vec{r})
\end{array}\right]=\left[\begin{array}{c}
S_{x x, \alpha}(\vec{r})+S_{y y, \alpha}(\vec{r}) \\
S_{x x, \alpha}(\vec{r})-S_{y y, \alpha}(\vec{r}) \\
S_{x y, \alpha}(\vec{r})+S_{y x, \alpha}(\vec{r}) \\
i\left[S_{x y, \alpha}(\vec{r})-S_{y x, \alpha}(\vec{r})\right]
\end{array}\right],} \\
\text { where } \quad S_{p q, \alpha}(\vec{r})=\iint_{\vec{\nu}_{\perp} \in \alpha} E_{\text {out }, p}\left(\vec{r}, \vec{\nu}_{\perp}\right) E_{\text {out }, q}^{*}\left(\vec{r}, \vec{\nu}_{\perp}\right) d^{2} \vec{\nu}_{\perp} .
\end{array}
$$

From here, we neglect the nonlinear contribution to the Stokes parameters from the scattering potential tensor, which is usually small for weakly scattering specimens, and arrive at our linearized forward model expressed in the Fourier space as

$$
\tilde{S}_{m, \alpha}^{\prime}(\vec{u})=\sum_{\substack{\ell=0 \mathrm{r}, 0 \mathrm{i}, 1 \mathrm{c}, 1 \mathrm{~s}, 2 \mathrm{c}, 2 \mathrm{~s}, 3}} \tilde{H}_{m, \ell, \alpha}(\vec{u}) \tilde{f}_{\ell}(\vec{u}) ; \quad m=0,1,2,3,
$$


where $\tilde{a}(\vec{u})$ denotes the Fourier transform of a function $a(\vec{r})$ at the 3D spatial frequency $\vec{u}=\left[\vec{u}_{\perp}^{T}, u_{z}\right]^{T}, \tilde{S}_{m, \alpha}^{\prime}(\vec{u})$ is the DCsubtracted Stokes parameter, $\tilde{H}_{m, \ell, \alpha}(\vec{u})$ is the transfer function mapping from each scattering potential tensor component $\ell$ to the $m$-th Stokes parameter under illumination pattern $\alpha$. In a 2D imaging case, the forward model only requires a simple modification of $u_{z}$-integration and is expressed as

$$
\begin{aligned}
& \tilde{S}_{m, \alpha}^{\prime}\left(\vec{u}_{\perp}, z=0\right)=\sum_{\substack{\ell=0 \mathrm{r}, 0 \mathrm{i}, 1 \mathrm{c}, 1 \mathrm{~s}, 2 \mathrm{c}, 2 \mathrm{~s}, 3}}\left[\int \tilde{H}_{m, \ell, \alpha}(\vec{u}) \cdot d u_{z}\right] \cdot \tilde{g}_{\ell}\left(\vec{u}_{\perp}\right) ; \quad m=0,1,2,3, \\
& \text { where } \quad \overline{\bar{g}}\left(\vec{r}_{\perp}\right)=\int_{z \in \mathrm{DOF}} \overline{\bar{f}}(\vec{r}) \cdot d z
\end{aligned}
$$

\section{B: Deconvolution algorithm}

Our deconvolution algorithm takes the Stokes parameters of the scattered light under different illuminations as inputs to estimate the physical properties that are encoded in the UPT. Since some parts of this algorithm are highly nonlinear, we decompose this algorithm into three parts for more robust estimation overall. The first part of the algorithm is a least square optimization solver to estimate the entries of the scattering potential tensor in 3D space. Secondly, we estimate the phase, principal retardance, and $3 \mathrm{D}$ orientation from the entries of the scattering potential tensor under positive and negative uniaxial material assumption. The last part of the algorithm takes these two sets of solution to estimate a probability of the material type throughout 3D space.

\section{B.1: Solving for scattering potential tensor}

According to the forward model Eq. (6), the DC-subtracted (background subtracted) Stokes parameters of the scattered light, $\tilde{S}_{m, \alpha}^{\prime}(\vec{u})$, under different illuminations are linearly related to the entries of the scattering potential tensors. Thus, we can write down an inverse problem to retrieve the entries of the scattering potential tensor as

$$
\min _{\tilde{f}_{\ell}(\vec{u})} \sum_{\alpha} \sum_{m=0}^{2}\left\|\tilde{S}_{m, \alpha}^{\prime}(\vec{u})-\sum_{\substack{\ell=0 \mathrm{r}, 0 \mathrm{i}, 1 \mathrm{c}, 1 \mathrm{~s}, 2 \mathrm{c}, 2 \mathrm{~s}, 3}} \tilde{H}_{m, \ell, \alpha}(\vec{u}) \tilde{f}_{\ell}(\vec{u})\right\|_{2}^{2}+\sum_{\substack{\ell=0 \mathrm{r}, 0 \mathrm{i}, 1 \mathrm{c}, 1 \mathrm{~s}, 2 \mathrm{c}, 2 \mathrm{~s}, 3}} \tau_{\ell}\left\|\tilde{f}_{\ell}(\vec{u})\right\|_{2}^{2},
$$

where $\tau_{\ell}$ is the Tikhonov regularization parameter for each independent term in the scattering potential tensor. In our implementation, this optimization is done in the Fourier space, where each voxel (or pixel) in Fourier space is independent to other voxels (or pixels). We then further decompose Eq. (6) into point-wise optimization by removing the dependence of $\vec{u}$ in the original optimization problem. The least square solution of this one-point optimization can be obtained by solving the fully determined inverse problem of

$$
\left[\left(\sum_{\alpha} \overline{\tilde{\tilde{H}}}_{\alpha}^{\dagger} \overline{\tilde{\tilde{H}}}_{\alpha}\right)+\operatorname{diag}(\vec{\tau})\right] \overrightarrow{\tilde{f}}=\overline{\overline{\mathcal{H}}} \overrightarrow{\tilde{f}}=\sum_{\alpha} \overline{\tilde{\tilde{H}}}_{\alpha}^{\dagger} \overrightarrow{\widetilde{S}}_{\alpha}^{\prime}
$$

where

$$
\begin{aligned}
& \overline{\overline{\tilde{H}}}_{\alpha}=\left[\begin{array}{ccccccc}
\tilde{H}_{0,0 \mathrm{r}, \alpha} & \tilde{H}_{0,0 \mathrm{i}, \alpha} & \tilde{H}_{0,1 \mathrm{c}, \alpha} & \tilde{H}_{0,1 \mathrm{~s}, \alpha} & \tilde{H}_{0,2 \mathrm{c}, \alpha} & \tilde{H}_{0,2 \mathrm{~s}, \alpha} & \tilde{H}_{0,3, \alpha} \\
\tilde{H}_{1,0 \mathrm{r}, \alpha} & \tilde{H}_{1,0 \mathrm{i}, \alpha} & \tilde{H}_{1,1 \mathrm{c}, \alpha} & \tilde{H}_{1,1 \mathrm{~s}, \alpha} & \tilde{H}_{1,2 \mathrm{c}, \alpha} & \tilde{H}_{1,2 \mathrm{~s}, \alpha} & \tilde{H}_{1,3, \alpha} \\
\tilde{H}_{2,0 \mathrm{r}, \alpha} & \tilde{H}_{2,0 \mathrm{i}, \alpha} & \tilde{H}_{2,1 \mathrm{c}, \alpha} & \tilde{H}_{2,1 \mathrm{~s}, \alpha} & \tilde{H}_{2,2 \mathrm{c}, \alpha} & \tilde{H}_{2,2 \mathrm{~s}, \alpha} & \tilde{H}_{2,3, \alpha}
\end{array}\right], \\
& \vec{\tau}=\left[\begin{array}{lllllll}
\tau_{0 \mathrm{r}} & \tau_{0 \mathrm{i}} & \tau_{1 \mathrm{c}} & \tau_{1 \mathrm{~s}} & \tau_{2 \mathrm{c}} & \tau_{2 \mathrm{~s}} & \tau_{3}
\end{array}\right]^{T}, \\
& \overrightarrow{\tilde{f}}=\left[\begin{array}{lllllll}
\tilde{f}_{0 \mathrm{r}} & \tilde{f}_{0 \mathrm{i}} & \tilde{f}_{1 \mathrm{c}} & \tilde{f}_{1 \mathrm{~s}} & \tilde{f}_{2 \mathrm{c}} & \tilde{f}_{2 \mathrm{~s}} & \tilde{f}_{3}
\end{array}\right]^{T}, \\
& \overrightarrow{\tilde{S}}_{\alpha}^{\prime}=\left[\begin{array}{lll}
\tilde{S}_{0, \alpha}^{\prime} & \tilde{S}_{1, \alpha}^{\prime} & \tilde{S}_{2, \alpha}^{\prime}
\end{array}\right]^{T}, \\
& \overline{\overline{\mathcal{H}}}=\left(\sum_{\alpha} \overline{\overline{\tilde{H}}}_{\alpha}^{\dagger} \overline{\overline{\tilde{H}}}_{\alpha}\right)+\operatorname{diag}(\vec{\tau}),
\end{aligned}
$$

$\overline{\tilde{\tilde{H}}}_{\alpha}^{\dagger}$ denotes the Hermitian of the matrix $\overline{\overline{\tilde{H}}}_{\alpha}$. With this fully determined inverse problem, we apply the Cramer's rule on it to obtain the analytical solution as

$$
\tilde{f}_{\ell}=\operatorname{det}\left(\overline{\overline{\mathcal{H}}}_{\ell}\right) / \operatorname{det}(\overline{\overline{\mathcal{H}}})
$$

where $\overline{\overline{\mathcal{H}}}_{\ell}$ denotes that the $\ell$-th column of the $\overline{\overline{\mathcal{H}}}$ matrix is replaced with $\sum_{\alpha} \overline{\overline{\tilde{H}}}_{\alpha}^{\dagger} \overrightarrow{\tilde{S}}_{\alpha}^{\prime}$. We set the regularization $\tau_{\ell}$ as a multiple of the mean absolute value of the $\ell$-th diagonal entry of $\overline{\overline{\mathcal{H}}}$ over all spatial frequency $\vec{u}$ to reduce its dependency on different 
imaging parameters. Usually the multiple factor of $\tau_{\ell}$ is ranging from 1 to 100 (smaller for less noisy data to get sharper reconstruction and larger for more noisy data to smooth out the noise). It should also be noted that, for simplicity of the math, we only show the single point solution of this deconvolution problem. In the actual implementation, we vectorize the whole 3D array of the data and the transfer functions for fast parallel processing of the data on GPU.

\section{B.2: Phase, principal retardance and 3D orientation from scattering potential tensor}

After solving Eq. (8), we obtain components of scattering potential tensor as shown in Eq. (3). $f_{0}(\vec{r})$ is related to the difference of average permittivity between the specimen and the environment, which corresponds to accumulated optical phase and absorption information. If the effect of phase and absorption is much stronger than the effect of optical anisotropy, $f_{0}(\vec{r})$ is roughly proportional to the phase and the absorption. If the permittivity of the environment is close to the average permittivity of the specimen, the phase and absorption are approximately related to $f_{0}(\vec{r})$ with the following scaling

$$
\begin{aligned}
\phi(\vec{r}) & \approx \frac{\Delta z}{2 k_{0} \sqrt{\epsilon_{\mathrm{rm}}}} \cdot \operatorname{Re}\left\{f_{0}(\vec{r})\right\} \\
\mu(\vec{r}) & \approx-\frac{\Delta z}{2 k_{0} \sqrt{\epsilon_{\mathrm{rm}}}} \cdot \operatorname{Im}\left\{f_{0}(\vec{r})\right\}
\end{aligned}
$$

where $\phi(\vec{r})$ and $\mu(\vec{r})$ are phase and absorption of the specimen and $\Delta z$ is the sampling size of our data in the axial direction.

Estimating principal retardance and 3D orientation from four terms in the scattering potential tensor, $f_{1 \mathrm{c}}(\vec{r}), f_{1 \mathrm{~s}}(\vec{r}), f_{2 \mathrm{c}}(\vec{r})$, $f_{2 \mathrm{~s}}(\vec{r})$, requires information of the optic sign $\left(n_{\mathrm{e}}>n_{\mathrm{o}}\right.$ or $\left.n_{\mathrm{e}}<n_{\mathrm{o}}\right)$. Here, we first provide two sets of analytical solution for principal retardance and 3D orientation under assumptions of two optic signs. These two sets of solution will be used to estimate the probability of the optic signs in the next part of algorithm. Using trigonometry relations, we express these two solutions of principal retardance and $3 \mathrm{D}$ orientation as

$$
\begin{aligned}
& \omega_{ \pm}(\vec{r})=\frac{1}{2} \tan ^{-1}\left[\frac{ \pm f_{1 \mathrm{~s}}(\vec{r})}{ \pm f_{1 \mathrm{c}}(\vec{r})}\right], \quad \text { when } n_{\mathrm{e}} \gtrless n_{\mathrm{o}} \\
& \theta_{ \pm}(\vec{r})=\tan ^{-1}\left\{\frac{ \pm 2\left[f_{1 \mathrm{~s}}(\vec{r}) \cdot \sin 2 \omega(\vec{r})+f_{1 \mathrm{c}}(\vec{r}) \cdot \cos 2 \omega(\vec{r})\right]}{ \pm\left[f_{2 \mathrm{~s}}(\vec{r}) \cdot \sin \omega(\vec{r})+f_{2 \mathrm{c}}(\vec{r}) \cdot \cos \omega(\vec{r})\right]}\right\}, \quad \text { when } n_{\mathrm{e}} \gtrless n_{\mathrm{o}} \\
& \rho_{ \pm}(\vec{r})=\frac{k_{0} \Delta z}{\sqrt{\epsilon_{\mathrm{rm}}}} \cdot \Delta \epsilon_{\mathrm{r}}(\vec{r})=\frac{\left[f_{1 \mathrm{~s}}(\vec{r}) \cdot \sin 2 \omega(\vec{r})+f_{1 \mathrm{c}}(\vec{r}) \cdot \cos 2 \omega(\vec{r})\right] \cdot \sin ^{2} \theta(\vec{r}) \cdot \Delta z}{k_{0} \sqrt{\epsilon_{\mathrm{rm}}} \cdot\left[\sin ^{4} \theta(\vec{r})+\sigma\right]},
\end{aligned}
$$

where $\omega_{ \pm}, \theta_{ \pm}$, and $\rho_{ \pm}$are in-plane orientation, out-of-plane inclination and principal retardance of the positive or negative uniaxial material. $\sigma$ is a small number to prevent noise amplification in the estimation of principal retardance. Typically, $\sigma=10^{-2} \sim 10^{-3}$ is a good choice to balance between accuracy and noise amplification.

At the end of this computation, the range of the azimuth is $\omega \in[0, \pi)$ and of the inclination is $\theta \in[0, \pi)$. These ranges correspond to the front half of the hemisphere of unit sphere $(y \geq 0)$. For intuitive visualization of 3D orientation, we transform $(\omega, \theta)$ coordinates to span the range of the top hemisphere (fig. $1 \mathrm{D}, z \geq 0)$ by reflecting the $3 \mathrm{D}$ orientations around the origin.

\section{B.3: Optic sign from scattering potential tensor}

The optic sign of the anisotropy reports symmetry of underlying structure. If we know of the type of the material imaged, we pick one set of solution from $\omega_{ \pm}, \theta_{ \pm}$, and $\rho_{ \pm}$. More often, the optic sign of a biological structure is not known and can be spatially variable.

For cases where the optic sign is unknown, here we describe an algorithm that models the scattering potential as a mixture of positive uniaxial and negative uniaxial material. The algorithm starts with constructing the scattering potential tensor components with positive $\left(\omega_{+}, \theta_{+}, \rho_{+}\right)$and negative $\left(\omega_{-}, \theta_{-}, \rho_{-}\right)$set of solutions according to Eq. (3). We then want to know which set of solution is more favorable in our data by solving the below optimization problem

$$
\min _{w_{+}(\vec{r}), w_{-}(\vec{r})} \sum_{\alpha} \sum_{m=0}^{2}\left\|\tilde{S}_{m, \alpha}^{\prime}(\vec{u})-\sum_{\substack{\ell=0 \mathrm{r}, 0 \mathrm{i}, 1 \mathrm{c}, 1 \mathrm{~s}, 2 \mathrm{c}, 2 \mathrm{~s}, 3}} \tilde{H}_{m, \ell, \alpha}(\vec{u}) \mathcal{F}\left\{w_{+}(\vec{r}) \cdot f_{\ell,+}(\vec{r})+w_{-}(\vec{r}) \cdot f_{\ell,-}(\vec{r})\right\}\right\|_{2}^{2},
$$

where $w_{+}(\vec{r})$ and $w_{-}(\vec{r})$ are the weights for positive and negative uniaxial solutions (we only consider positive values of the weight). When the positive material is more favorable (the structure within a voxel is denser along axis of symmetry), $w_{+}(\vec{r})$ is larger than $w_{-}(\vec{r})$. On the other hand, $w_{-}(\vec{r})$ is larger than $w_{+}(\vec{r})$ when negative material is more favorable (the structure is denser perpendicular to the symmetry axis). When the material is isotropic, $w_{+}(\vec{r}) \approx w_{-}(\vec{r})$. We implement a gradient descent iterative algorithm to solve this optimization. To identify material type with these two weights, we define the probability of 
being positive material to be

$$
p_{+}(\vec{r})=\frac{\max \left[\frac{w_{+}(\vec{r})}{\left|w_{+}(\vec{r})\right|+\left|w_{-}(\vec{r})\right|}, w_{c}\right]}{\max \left[\frac{w_{+}(\vec{r})}{\left|w_{+}(\vec{r})\right|+\left|w_{-}(\vec{r})\right|}, w_{c}\right]+\max \left[\frac{w_{-}(\vec{r})}{\left|w_{+}(\vec{r})\right|+\left|w_{-}(\vec{r})\right|}, w_{c}\right]},
$$

where $w_{c}$ is a cut-off weight to threshold out noisy weight estimate for smooth probability reconstruction. $w_{c}$ is usually set around $0.05 \sim 0.2$. The higher the value, the stronger the thresholding effect.

\section{C: Intensity to Stokes parameters}

In fig. 1, we have demonstrated that the raw images from uPTI setup contain intensity modulation induced from specimen's permittivity tensor. We have also established a forward model to describe the relationship between components of specimen's permittivity tensor and the Stokes parameters of the output scattered light. Here we connect the missing link between the Stokes parameters of the scattered light to our measured intensity for further permittivity tensor retrieval.

\section{C.1: Calibration}

Ideally, our polarization sensitive camera perfectly detects four channels of linearly polarized light oriented at $0^{\circ}, 45^{\circ}, 90^{\circ}$, and $135^{\circ}$. According to the definition of the Stokes vector (76), the measured intensity is linearly related to the Stokes parameters in the following form

$$
\vec{I}=\left[\begin{array}{c}
I_{0} \\
I_{45} \\
I_{90} \\
I_{135}
\end{array}\right]=\frac{1}{2}\left[\begin{array}{cccc}
1 & 1 & 0 & 0 \\
1 & 0 & 1 & 0 \\
1 & -1 & 0 & 0 \\
1 & 0 & -1 & 0
\end{array}\right] \cdot\left[\begin{array}{c}
S_{0} \\
S_{1} \\
S_{2} \\
S_{3}
\end{array}\right]=\overline{\bar{A}} \cdot \vec{S}
$$

where $\vec{I}$ is the intensity vector of each polarization measurement, $\overline{\bar{A}}$ is the instrument matrix (47) of the optical system, and $\vec{S}$ is Stokes vector of the light. Applying the inverse of the known instrument matrix, $\overline{\bar{A}}$, on the measured intensity vector, $\vec{I}$, allows us to get the first three Stokes parameters for further deconvolution. However, the actual optical setup has multiple components (e.g. beam splitting prisms) with unexpected polarization effects. Such components provide non-ideal values in the instrument matrix. Thus, a calibration procedure to measure the instrument matrix is essential. To calibrate the unknown instrument matrix, we adopt the approach in (47). We place a linear polarizer mounted on a rotational stage at the specimen plane and rotate it to collect corresponding intensity variations in four polarization channel. According to Mueller matrix formulation, we model the intensities collected at angle $\omega_{\mathrm{LP}}$ of the linear polarizer and retrieve the instrument matrix. Additional to the previous method, we demonstrate extra calibration of the polarization state of the incident light.

Here we show how we model our calibration through Mueller formulation. Assuming the incident light has the Stokes parameters of $\vec{S}_{s}=\left[S_{s 0}, S_{s 1}, S_{s 2}, S_{s 3}\right]^{T}$, the generated polarization state of light after the linear polarizer aligned with angle $\omega_{\mathrm{LP}}$ in Stokes parameters is calculated as

$$
\begin{aligned}
\vec{S}_{\mathrm{LP}}\left(\omega_{\mathrm{LP}}\right) & =\overline{\bar{M}}_{\mathrm{LP}}\left(\omega_{\mathrm{LP}}\right) \cdot \vec{S}_{s}=S_{\mathrm{LP}, 0}\left(\omega_{\mathrm{LP}}\right) \cdot\left[\begin{array}{c}
1 \\
\cos 2 \omega_{\mathrm{LP}} \\
\sin 2 \omega_{\mathrm{LP}} \\
0
\end{array}\right], \\
\text { where } S_{\mathrm{LP}, 0}\left(\omega_{\mathrm{LP}}\right) & =S_{s 0}+S_{s 1} \cdot \cos 2 \omega_{\mathrm{LP}}+S_{s 2} \cdot \sin 2 \omega_{\mathrm{LP}}
\end{aligned}
$$

$\overline{\bar{M}}_{\mathrm{LP}}(\alpha)$ is the Mueller matrix of a linear polarizer aligned at angle $\omega_{\mathrm{LP}}$. This formula shows that the total energy of the generated state, $S_{\mathrm{LP}, 0}\left(\omega_{\mathrm{LP}}\right)$, will be a constant along $\omega_{\mathrm{LP}}$ if the input light is unpolarized or circularly polarized. This total energy can vary if our input light has some linear polarization components. We estimate the total energy of the generated light by summing over intensities of four polarization channel. Through a Fourier series fitting, we obtain the Stokes parameters of the incident light and use it for later deconvolution.

Sending this generated polarization state into the detection optical system, we get the measured intensity through the multiplication of the instrument matrix on the generated Stokes parameters

$$
\vec{I}\left(\omega_{\mathrm{LP}}\right)=\overline{\bar{A}} \vec{S}_{\mathrm{LP}}\left(\omega_{\mathrm{LP}}\right)=S_{\mathrm{LP}, 0}\left(\omega_{\mathrm{LP}}\right) \cdot\left(\vec{a}_{0}+\vec{a}_{1} \cdot \cos 2 \omega_{\mathrm{LP}}+\vec{a}_{2} \cdot \sin 2 \omega_{\mathrm{LP}}\right)
$$

where $\vec{a}_{i}$ is the $i+1$-th column of the instrument matrix $\overline{\bar{A}}$. If we normalize the measured intensity with the total energy of the generated light, $S_{\mathrm{LP}, 0}\left(\omega_{\mathrm{LP}}\right)$, we observe that the first three columns of instrument matrix are the Fourier series coefficients 
of the normalized intensities along $\omega_{\mathrm{LP}}$. We conduct another fitting to estimate our instrument matrix. With the first three columns of the instrument matrix, we then used it to obtain the first three Stokes parameters of the light as

$$
\left[\begin{array}{l}
S_{0} \\
S_{1} \\
S_{2}
\end{array}\right]=\left[\begin{array}{lll}
\vec{a}_{0} & \vec{a}_{1} & \vec{a}_{2}
\end{array}\right]^{+} \vec{I}
$$

where $A^{+}$denotes a pseudo-inverse of the matrix $A$.

\section{C.2: Background correction}

This Stokes parameters may still subject to leftover polarization effects that produce slowly varying background. In addition, the deconvolution works with the DC-subtracted Stokes parameters according to Eq. (6). With the derivation described in the Supplementary Note 2, we obtain the DC-subtracted Stokes parameters with the following operations

$$
\left[\begin{array}{l}
S_{0}^{\prime} \\
S_{1}^{\prime} \\
S_{2}^{\prime}
\end{array}\right]=\left[\begin{array}{l}
S_{0} \\
S_{1} \\
S_{2}
\end{array}\right] \cdot \frac{1}{S_{\mathrm{bg}, 0}}-\left[\begin{array}{c}
0 \\
S_{\mathrm{bg}, 1} \\
S_{\mathrm{bg}, 2}
\end{array}\right] \cdot \frac{S_{0}}{S_{\mathrm{bg}, 0}^{2}}-\left[\begin{array}{l}
1 \\
0 \\
0
\end{array}\right],
$$

where $S_{\mathrm{bg}, \mathrm{i}}$ is the $i$-th background Stokes parameters obtained when imaging at an empty field of view. When the transmission modulation of the specimen is relatively weak, $S_{0} / S_{\mathrm{bg}, 0} \approx 1$ and this is simply removing the DC components of the Stokes parameters. These operations are done to all sets of polarization acquisitions with different illumination patterns and then the deconvolution takes these DC-subtracted Stokes parameters for the retrieval of uniaxial permittivity tensor components.

\section{D: Transfer functions and choice of illumination pattern}

The choice of the illumination patterns for our experiment is modified from past literature of 3D diffrential phase contrast (3D DPC) microscopy (35) and 3D polarized light imaging (3D PLI) (38-40). To get 3D phase information, 3D DPC exploits illumination patterns of 4 rotating half-circles with NA matched in illumination and detection, which is an extension from 2D DPC (34). In 3D PLI, 3D orientation and the principal retardance of the optically anisotropic material (axons in human brain) are extracted with brightfield illumination, polarization-sensitive detection, and a tilting stage. In their acquisition, one untilted condition followed by four tilted conditions in $90^{\circ}$-separated orientations are used. The 4 tilted conditions with brightfield illumination is similar to untilted stage illuminating with 4 off-axis illuminations in rotating orientations as what it has been done in 3D DPC. The one untilted polarization measurement plays an important role for 3D orientation retrieval as shown in (38). From these rules of thumb, it is reasonable to use combination of brightfield illumination pattern plus several offaxis illumination patterns in a polarization-sensitive detection system for acquiring 3D phase, principal retardance, and 3D orientation information.

There are multiple combinations of patterns that could be used to get the information of permittivity tensor. Different illumination patterns result in different shapes of the transfer function in Eq. (6), which affects noise robustness over 3D spatial frequencies. By visualizing these transfer functions, we get a sense of how well specific information is transferred with the illumination patterns. Figure 1-supplement 2 compares the absolute sums of the transfer functions over illumination patterns for two possible combinations of illumination patterns that are deduced from past literature, which describe how individual scattering potential tensor components couple into the measured intensities as a function of their spatial frequencies. In summary, $S_{0}$ carries phase $(\ell=0 \mathrm{r})$ and absorption information $(\ell=0 \mathrm{i})$, while $S_{1}$ and $S_{2}$ carry anisotropy information $(\ell=1 \mathrm{c}, 1 \mathrm{~s}, 2 \mathrm{c}, 2 \mathrm{~s}, 3)$. For both sets of illumination patterns, phase, absorption, and projected anisotropy information $(\ell=1 \mathrm{c}, 1 \mathrm{~s})$ transfer relatively well compared to the last three components $(\ell=2 \mathrm{c}, 2 \mathrm{~s}, 3)$. The last component of the scattering potential tensor, which contains information of optic sign, is the most poorly-transferred in both cases. This is why the optic sign estimation is noisy. Comparing the energy of the transfer function with these two sets of illumination, we find that the second set of illumination patterns generates more uniform energy of transfer function for all the scattering potential components. Offaxis illumination with more orientations tends to give better information transfer and better noise robustness across different spatial frequencies, but it takes more time for each experiment. To balance the trade-off between the noise performance and the acquisition time, we choose the second set of illumination patterns for our experiment, which is 8 off-axis max-NA sector patterns followed by 1 on-axis half-NA brightfield pattern. Note that these are not the only choices for proper permittivity tensor retrieval. Other combinations of patterns are possible, but it would require further investigation to determine the optimal one as that is done in phase imaging community $(77,78)$.

\section{E: Computation of 3D orientations of axons and their cross-sections from structure tensor of phase image}

To obtain the structure tensor for analysis of axons' morphological orientation, we consult and modify the vessel segmentation algorithm from the X-ray angiography community (79) for our data shown in fig. 4B. The algorithm first computes the structure 
tensor, the second-order gradient, of the phase image $\phi(\vec{r})$, as

$$
\mathcal{H}(\vec{r}, s)=\left[\begin{array}{ccc}
\partial_{x, s}^{2} & \partial_{y, s} \partial_{x, s} & \partial_{z, s} \partial_{x, s} \\
\partial_{x, s} \partial_{y, s} & \partial_{y, s}^{2} & \partial_{z, s} \partial_{y, s} \\
\partial_{x, s} \partial_{z, s} & \partial_{y, s} \partial_{z, s} & \partial_{z, s}^{2}
\end{array}\right] \phi(\vec{r}),
$$

where $s$ is the scale parameter and scale-dependent gradient is defined as a convolution between the scaled intensity and the derivative of a Gaussian function written as

$$
\partial_{i, s} I(\vec{r})=[s I(\vec{r})] \otimes_{\vec{r}}\left\{\partial_{i}\left[\left(2 \pi s^{2}\right)^{-3 / 2} \cdot e^{-\|\vec{r}\|^{2} / 2 s^{2}}\right]\right\} .
$$

Depending on the geometry of the interested feature (a shell-like, tubular-like, or blob-like structues), the eigenvalues of the structure tensor may be used in different ways to construct the segmentation map. In our case, we targeted shell-like structure and construct the segmentation map as

$$
\mathcal{V}(\vec{r})=\left\{\begin{array}{c}
\left(1-e^{-\mathcal{R}(\vec{r}) / 2 a^{2}}\right) \cdot\left(1-e^{-\mathcal{S}(\vec{r}) / 2 b^{2}}\right), \\
0, \quad \zeta_{2}(\vec{r})>0
\end{array}\right.
$$

where

$$
\begin{aligned}
& \mathcal{R}(\vec{r})=\frac{\left|\zeta_{2}(\vec{r})\right|}{\sqrt{\zeta_{0}(\vec{r}) \cdot \zeta_{1}(\vec{r})}} \\
& \mathcal{S}(\vec{r})=\sqrt{\sum_{i \leq 2} \zeta_{i}^{2}(\vec{r})},
\end{aligned}
$$

$\left|\zeta_{2}(\vec{r})\right|>\left|\zeta_{1}(\vec{r})\right|>\left|\zeta_{0}(\vec{r})\right|$ are eigenvalues of the structure tensor at position $\vec{r}, a$ and $b$ are parameters to adjust the level of sharpness of the segmentation map. The eigenvectors corresponding to the largest eigenvalue of the structure tensor on every voxel within this map contain the normal orientation of the shell-like structures. We then use these eigenvectors as a different measurement of lipids' 3D orientation to verify our results from 3D anisotropy.

\section{F: Specimen preparation}

\section{Femtosecond laser written anisotropic glass}

The target used in fig. 2 was written into a fused silica cover glass that is about $0.25 \mathrm{~mm}$ thick and $22 \mathrm{~mm}$ by $22 \mathrm{~mm}$ on its sides using a polarized femtosecond laser. The star pattern consists of 32 equally spaced birefringent wedges that rotate in steps of $11.25^{\circ}$. The wedges consist of a single line near the center of the star, flanked by additional lines towards larger diameters ( 1 line between 3 and $20 \mu \mathrm{m}$ diameter, 3 lines between 20 and $40 \mu \mathrm{m}$, and 5 lines between 40 and $60 \mu \mathrm{m}$ ). While the slow axis of a wedge rotated with the wedge, within a wedge, the slow axis was uniform and was parallel to the lines. Each line was written only once and the scanning direction was parallel to the slow axis. The parameters of laser fabrication were the following: pulse duration $-500 \mathrm{fs}$, repetition rate $-500 \mathrm{kHz}$, fabrication speed $-0.01 \mathrm{~mm} / \mathrm{s}$, wavelength $-515 \mathrm{~nm}$, focused with $0.55 \mathrm{NA}$ lens.

\section{F.1: Mouse brain section}

The mice were anesthetized by inhalation of isoflurane in a chemical fume hood and then perfused with $25 \mathrm{ml}$ phosphatebuffered saline (PBS) into the left cardiac ventricle and subsequently with $25 \mathrm{ml}$ of $4 \%$ paraformaldehyde (PFA) in the PBS solution. Thereafter, the brains were post-fixed with $4 \%$ PFA for 12-16 hours and then transferred to $30 \%$ sucrose solution at the temperature of $4^{\circ} \mathrm{C}$ for 2-3 days until the tissue sank to the bottom of the container. Then, the brains were embedded in a tissue freezing medium (Tissue-Tek O.C.T compound 4583, Sakura) and kept at the temperature of $-80^{\circ} \mathrm{C}$. Cryostat-microtome (Leica CM 1850, Huston TX) was used for preparing the tissue sections $(12$ and $50 \mu \mathrm{m})$ at the temperature of $-20^{\circ} \mathrm{C}$ and the slides were stored at the temperature of $-20^{\circ} \mathrm{C}$ until use. Upon experiment, the OCT on the slides were melted by keeping the slides at $37^{\circ} \mathrm{C}$ for $15-30$ minutes. Then, the slides were washed in PBST (PBS+Tween-20 [0.1\%]) for five minutes and then washed in PBS for five minutes and coversliped by mounting media (F4680, FluromountTM aqueousm sigma).

\section{iPSC cardiomyocyte}

Cardiomyocytes were differentiated from iPS cells (WTc cell line (80)) using a modified Wnt pathway modulation protocol (81). Briefly, cells were maintained in mTesr media (Stem Cell Technologies) and three days before differentiation, they were seeded on 12-well plates. During differentiation, basal media was RPMI supplemented with B-27 minus insulin (Gibco) for days 0-7 and RPMI with B-27 (Gibco) on days 7-onwards. Cells were treated with $6 \mu \mathrm{M}$ CHIR99021 (Tocris) for 48 hours on day 0 , and with $5 \mu \mathrm{M}$ IWP2 (Tocris) for 48 hours on day 3 . At day 15 , cells were harvested and stored on cryovials. 
bioRxiv preprint doi: https://doi.org/10.1101/2020.12.15.422951; this version posted December 16, 2020. The copyright holder for this preprint (which was not certified by peer review) is the author/funder, who has granted bioRxiv a license to display the preprint in perpetuity. It is made available under aCC-BY-NC-ND 4.0 International license.

$\mathrm{G}$ Automation for multi-scale imaging and analysis

When ready for experiments, cell pools were thawed in RPMI with B-27 supplemented with 20\% FBS (HyClone) and ROCK inhibitor Y-27632 (10 $\mu \mathrm{M}$, Selleckchem). Cardiomyocytes were then selected in culture using a metabolic switch method (82) by treating the cells with $4 \mathrm{mM}$ lactate media changes every other day for 6 days. Final cultures were $>90 \%$ ACTN+.

On day 30, cardiomyocytes were replated into glass coverslips, and maintained on RPMI with B-27 for five more days. Then they were fixed using $4 \%$ paraformaldehyde for 20 minutes at room temperature, washed three times with PBS supplemented with Triton X-100 (PBS-T) and blocked and permeabilized with 5\% bovine serum albumin in PBS-T. Cells were then stained with an anti-cTnT antibody (Abcam, ab45932) in PBS-T overnight, and with DAPI for 10 minutes. After three PBS-T washes, Alexa Fluor 488 anti-rabbit (ThermoFisher) in PBS-T was used as secondary antibody, followed by three more PBS-T washes. Then, a drop of Prolong Antifade (without DAPI) (ThermoFisher) was added to coverslips for mounting into a glass slide.

\section{G: Automation for multi-scale imaging and analysis}

We automate the multi-scale imaging shown in fig. 3 and Video 3 by controlling individual devices in python. This acquisition requires control of three main devices, the LCD panel for switching illumination patterns, the machine vision polarization camera for collecting images, and the microscope stages for scanning in $x, y$, and $z$ directions. First, we control the LCD panel using the built-in APIs from adafruit with Arduino board. Serial connection is established from the acquisition computer to the arduino board for software triggering in python. Second, we control the polarization camera with a python package, PySpin, developed by the provider of the camera, FLIR. Last, the microscope stage is controlled by Micro-Manager (https: //github.com/micro-manager). In order to build a bridge between the Java-based Micro-Manager and python, we leverage mm2python library (https://github.com/czbiohub/mm2python). Collectively, these packages allow us to compose an acquisition script to control each device to function at the right moment. For the $2 \mathrm{D}$ acquisition shown in fig. 3A3B, we acquired 9 images under different illumination patterns per location for total 609 FOVs in a $29 \times 21(x \times y)$ rectangular grid. The overlap between each location is set to be $\sim 15 \%$ in $x$ direction and $\sim 30 \%$ in $y$ direction. For 3D acquistion shown in fig. 3C-3I and Video 3, we acquired $9 \times 120$ (pattern $\times z$ ) images to form a $z$-stack per location for a total 153 FOVs in a $17 \times 9(x \times y)$ rectangular grid. The overlap parameter is similar to the previous case.

We implement our algorithm to be GPU-compatible on an IBM Power9 server equipped with 4 GPUs (Tesla V100-SXM232GB, NVIDIA) per compute node. One FOV of 2D acquisition easily fits in the memory of one GPU, so we initiate 4 instances of computation with 4 different GPU to process the data. The algorithm takes about 200 seconds to process one FOV of 2D acquisition and about half an hour to stitch one channel of the reconstruction. Fitting a FOV of 3D acquisition in the memory of one GPU is infeasible, so we break one 3D acquisition into 30 (or smaller number) small patches for processing. We also intiate 4 to 8 instances of computation with 4 GPUs to process these small patches in parallel. Each FOV of 3D acquisition takes about 6 hours of the processing time and the stitching process takes about 3 hours for one channel of the reconstruction.

\section{Acknowledgements}

We thank Rafael Gómez-Sjöberg, CZ Biohub, for critical reading of the manuscript. L-H.Y, I.E.I, B.C, S-M.G, J.R.B, and S.B.M are supported by the intramural program of the Chan Zuckerberg Biohub. B.R.C received support from the Gladstone Institutes, Innovative Genomics Institute and NIH grants R01-HL130533, R01-HL13535801, P01-HL146366. This research is supported by the Chan Zuckerberg Biohub.

\section{Competing Interests Statement}

A patent filed by the Chan Zuckerberg Biohub with S.B.M, L-H.Y, and I.E.I as inventors is pending and describes the uPTI method reported in this paper. B.R.C. is a founder of Tenaya Therapeutics (https://www.tenayatherapeutics.com/), a company focused on finding treatments for heart failure, including genetic cardiomyopathies. Other authors declare no competing interests.

\section{References}

1. Z. Zheng, J. S. Lauritzen, E. Perlman, C. G. Robinson, M. Nichols, D. Milkie, O. Torrens, J. Price, C. B. Fisher, N. Sharifi, S. A. Calle-Schuler, L. Kmecova, I. J. Ali, B. Karsh, E. T. Trautman, J. A. Bogovic, P. Hanslovsky, G. S. X. E. Jefferis, M. Kazhdan, K. Khairy, S. Saalfeld, R. D. Fetter, and D. D. Bock, "A Complete Electron Microscopy Volume of the Brain of Adult Drosophila melanogaster," Cell, vol. 174, no. 3, pp. 730-743.e22, Jul. 2018.

2. B. L. Edlow, A. Mareyam, A. Horn, J. R. Polimeni, T. Witzel, M. D. Tisdall, J. C. Augustinack, J. P. Stockmann, B. R. Diamond, A. Stevens, L. S. Tirrell, R. D. Folkerth, L. L. Wald, B. Fischl, and A. van der Kouwe, "7 Tesla MRI of the ex vivo human brain at 100 micron resolution," Scientific Data, vol. 6, no. 1, pp. 1-10, Oct. 2019.

3. M. Born and E. Wolf, Principles of Optics: Electromagnetic Theory of Propagation, Interference and Diffraction of Light. Elsevier, Jun. 2013.

4. S. Lee, H. Park, K. Kim, Y. Sohn, S. Jang, and Y. Park, "Refractive index tomograms and dynamic membrane fluctuations of red blood cells from patients with diabetes mellitus," Scientific Reports, vol. 7, no. 1, p. 1039, Apr. 2017.

5. R. Imai, T. Nozaki, T. Tani, K. Kaizu, K. Hibino, S. Ide, S. Tamura, K. Takahashi, M. Shribak, and K. Maeshima, "Density imaging of heterochromatin in live cells using orientation-independent-DIC microscopy," Molecular Biology of the Cell, vol. 28, no. 23, pp. 3349-3359, Aug. 2017.

6. P. A. Sandoz, C. Tremblay, F. G. van der Goot, and M. Frechin, "Image-based analysis of living mammalian cells using label-free 3D refractive index maps reveals new organelle dynamics and dry mass flux," PLOS Biology, vol. 17, no. 12, p. e3000553, Dec. 2019.

7. H. V. Pham, L. Pantanowitz, and Y. Liu, "Quantitative phase imaging to improve the diagnostic accuracy of urine cytology," Cancer Cytopathology, vol. 124, no. 9, pp. 641-650, 2016.

8. S. Inoué, "Polarization optical studies of the mitotic spindle," Chromosoma, vol. 5, no. 1, pp. 487-500, Dec. 1953.

9. J. Brugués and D. Needleman, "Physical basis of spindle self-organization," Proceedings of the National Academy of Sciences, vol. 111, no. 52, pp. 18496-18500, Dec. 2014. 
bioRxiv preprint doi: https://doi.org/10.1101/2020.12.15.422951; this version posted December 16, 2020. The copyright holder for this preprint (which was not certified by peer review) is the author/funder, who has granted bioRxiv a license to display the preprint in perpetuity. It is made available under aCC-BY-NC-ND 4.0 International license.

10. M. Axer, D. Grässel, M. Kleiner, J. Dammers, T. Dickscheid, J. Reckfort, T. Hütz, B. Eiben, U. Pietrzyk, K. Zilles, and K. Amunts, "High-resolution fiber tract reconstruction in the human brain by means of three-dimensional polarized light imaging," Frontiers in Neuroinformatics, vol. 5, Dec. 2011

11. H. Axer, S. Beck, M. Axer, F. Schuchardt, J. Heepe, A. Flücken, M. Axer, A. Prescher, and O. W. Witte, "Microstructural analysis of human white matter architecture using polarized light imaging: Views from neuroanatomy," Frontiers in Neuroinformatics, vol. 5, 2011.

12. M. Menzel, J. Reckfort, D. Weigand, H. Köse, K. Amunts, and M. Axer, "Diattenuation of brain tissue and its impact on 3D polarized light imaging," arXiv:1703.04343 [physics], Mar. 2017.

13. J. Mollink, M. Kleinnijenhuis, A.-M. van Cappellen van Walsum, S. N. Sotiropoulos, M. Cottaar, C. Mirfin, M. P. Heinrich, M. Jenkinson, M. Pallebage-Gamarallage, O. Ansorge, S. Jbabdi, and K. L. Miller, "Evaluating fibre orientation dispersion in white matter: Comparison of diffusion MRI, histology and polarized light imaging," Neurolmage, vol. 157, pp. 561-574, Aug. 2017.

14. M. M. Zeineh, N. Palomero-Gallagher, M. Axer, D. Gräßel, M. Goubran, A. Wree, R. Woods, K. Amunts, and K. Zilles, "Direct Visualization and Mapping of the Spatial Course of Fiber Tracts at Microscopic Resolution in the Human Hippocampus," Cerebral Cortex, vol. 27, no. 3, pp. 1779-1794, Mar. 2017.

15. D. J. H. A. Henssen, J. Mollink, E. Kurt, R. van Dongen, R. H. M. A. Bartels, D. Grä $\beta$ el, T. Kozicz, M. Axer, and A.-M. Van Cappellen van Walsum, "Ex vivo visualization of the trigeminal pathways in the human brainstem using 11.7T diffusion MRI combined with microscopy polarized light imaging," Brain Structure and Function, vol. 224, no. 1, pp. 159-170, Jan. 2019.

16. N.-J. Jan, K. Lathrop, and I. A. Sigal, "Collagen Architecture of the Posterior Pole: High-Resolution Wide Field of View Visualization and Analysis Using Polarized Light Microscopy," Investigative Ophthalmology \& Visual Science, vol. 58, no. 2, pp. 735-744, Feb. 2017.

17. B. Yang, N.-J. Jan, B. Brazile, A. Voorhees, K. L. Lathrop, and I. A. Sigal, "Polarized light microscopy for 3-dimensional mapping of collagen fiber architecture in ocular tissues," Journal of Biophotonics, vol. 11, no. 8, p. e201700356, 2018.

18. E. Bricchi, B. G. Klappauf, and P. G. Kazansky, "Form birefringence and negative index change created by femtosecond direct writing in transparent materials," Optics Letters, vol. 29, no. 1, pp. 119-121, Jan. 2004.

19. M. Sakakura, Y. Lei, L. Wang, Y.-H. Yu, and P. G. Kazansky, "Ultralow-loss geometric phase and polarization shaping by ultrafast laser writing in silica glass," Light: Science \& Applications, vol. 9 , no. 1, p. 15, Feb. 2020.

20. Y. Cotte, F. Toy, P. Jourdain, N. Pavillon, D. Boss, P. Magistretti, P. Marquet, and C. Depeursinge, "Marker-free phase nanoscopy," Nature Photonics, vol. 7, no. 2, pp. 113-117, Feb. 2013.

21. Y. Park, S. Shin, and G. S. Park, "3d refractive index tomography and structured illumination microscopy system using wavefront shaper and method thereof," EP Patent EP3 255414 A1, Dec., 2017.

22. T. H. Nguyen, M. E. Kandel, M. Rubessa, M. B. Wheeler, and G. Popescu, "Gradient light interference microscopy for 3D imaging of unlabeled specimens," Nature Communications, vol. 8, no. 1, p. 210, Dec. 2017

23. M. I. Shribak, J. LaFountain, D. S. C. Biggs, and S. Inoue, "Orientation-independent differential interference contrast microscopy and its combination with an orientation-independent polarization system," Journal of Biomedical Optics, vol. 13, no. 1, p. 014011, Jan. 2008

24. P. Ferrand, A. Baroni, M. Allain, and V. Chamard, "Quantitative imaging of anisotropic material properties with vectorial ptychography," Optics Letters, vol. 43, no. 4, p. 763, Feb. 2018.

25. A. Baroni, V. Chamard, and P. Ferrand, "Extending quantitative phase imaging to polarization-sensitive materials," Physical Review Applied, vol. 13, no. 5, p. 054028, May 2020.

26. N. Streibl, "Three-dimensional imaging by a microscope," JOSA A, vol. 2, no. 2, pp. 121-127, Feb. 1985.

27. L. Waller, L. Tian, and G. Barbastathis, "Transport of intensity phase-amplitude imaging with higher order intensity derivatives," Optics express, vol. 18, no. 12, pp. 12552-12561, 2010.

28. J. M. Soto, J. A. Rodrigo, and T. Alieva, "Label-free quantitative 3D tomographic imaging for partially coherent light microscopy," Optics Express, vol. 25, no. 14, pp. 15699-15712, Jul. 2017.

29. R. Oldenbourg and G. Mei, "New polarized light microscope with precision universal compensator," Journal of Microscopy, vol. 180, no. 2, pp. 140-147, 1995.

30. M. Shribak and R. Oldenbourg, "Techniques for fast and sensitive measurements of two-dimensional birefringence distributions," Applied Optics, vol. 42, no. 16, pp. 3009-3017, Jun. 2003.

31. S. B. Mehta, M. Shribak, and R. Oldenbourg, "Polarized light imaging of birefringence and diattenuation at high resolution and high sensitivity," Journal of Optics, vol. 15, no. 9 , p. 094007, Sep. 2013.

32. S.-M. Guo, L.-H. Yeh, J. Folkesson, I. E. Ivanov, A. P. Krishnan, M. G. Keefe, E. Hashemi, D. Shin, B. B. Chhun, N. H. Cho, M. D. Leonetti, M. H. Han, T. Nowakowski, and S. B. Mehta, "Revealing architectural order with quantitative label-free imaging and deep learning," eLife, vol. 9, p. e55502, Jul. 2020.

33. S. B. Mehta and C. J. R. Sheppard, "Quantitative phase-gradient imaging at high resolution with asymmetric illumination-based differential phase contrast," Optics Letters, vol. 34 , no. 13, pp. 1924-1926, 2009.

34. L. Tian and L. Waller, "Quantitative differential phase contrast imaging in an LED array microscope," Optics Express, vol. 23, no. 9, pp. 11394-11 403, May 2015.

35. M. Chen, L. Tian, and L. Waller, "3D differential phase contrast microscopy," Biomedical Optics Express, vol. 7, no. 10, pp. 3940-3950, Oct. 2016.

36. H.-H. Chen, Y.-Z. Lin, and Y. Luo, "Isotropic differential phase contrast microscopy for quantitative phase bio-imaging," Journal of Biophotonics, vol. 11, no. 8, p. e201700364, 2018.

37. W. Kaminsky, E. Gunn, R. Sours, and B. Kahr, "Simultaneous false-colour imaging of birefringence, extinction and transmittance at camera speed," Journal of Microscopy, vol. 228, no. 2, pp. 153-164, 2007.

38. H. Wiese, "Enhancing the Signal Interpretation and Microscopical Hardware Concept of 3D Polarized Light Imaging," Wissenschaftliche Abschlussarbeiten " Dissertation, Universität Wuppertal, Fakultät für Mathematik und Naturwissenschaften » Physik » Dissertationen, Jan. 2018.

39. D. Schmitz, S. E. A. Muenzing, M. Schober, N. Schubert, M. Minnerop, T. Lippert, K. Amunts, and M. Axer, "Derivation of fiber orientations from oblique views through human brain sections in 3D-polarized light imaging," Frontiers in Neuroanatomy, vol. 12, 2018

40. D. Schmitz, K. Amunts, T. Lippert, and M. Axer, "A least squares approach for the reconstruction of nerve fiber orientations from tiltable specimen experiments in 3D-PLI," in 2018 IEEE 15 th International Symposium on Biomedical Imaging (ISBI 2018), Apr. 2018, pp. 132-135.

41. S. B. Mehta, M. McQuilken, P. J. L. Riviere, P. Occhipinti, A. Verma, R. Oldenbourg, A. S. Gladfelter, and T. Tani, "Dissection of molecular assembly dynamics by tracking orientation and position of single molecules in live cells," Proceedings of the National Academy of Sciences, vol. 113, no. 42, pp. E6352-E6361, Oct. 2016.

42. M. Koike-Tani, T. Tominaga, R. Oldenbourg, and T. Tani, "Instantaneous polarized light imaging reveals activity dependent structural changes of dendrites in mouse hippocampal slices," bioRxiv, p. 523571,2019

43. S.-T. Wu, R. Voltoline, and C. L. Yasuda, "A view-independent line-coding colormap for diffusion tensor imaging," Computers \& Graphics, vol. 60, pp. 66-75, Nov. 2016.

44. A. L. Alexander, J. E. Lee, M. Lazar, and A. S. Field, "Diffusion tensor imaging of the brain," Neurotherapeutics, vol. 4, no. 3, pp. 316-329, Jul. 2007.

45. P. Müller, M. Schürmann, and J. Guck, "The Theory of Diffraction Tomography," arXiv:1507.00466 [physics, q-bio], Jul. 2015.

46. B. Ge, "Quantitative anisotropy imaging with polarized interference microscopy," Thesis, Massachusetts Institute of Technology, 2018.

47. R. M. A. Azzam and A. G. Lopez, "Accurate calibration of the four-detector photopolarimeter with imperfect polarizing optical elements," Journal of the Optical Society of America A, vol. 6, no. 10, p. 1513, Oct. 1989

48. R. Oldenbourg, "Analysis of edge birefringence," Biophysical Journal, vol. 60, no. 3, pp. 629-641, Sep. 1991.

49. C. Corbari, A. Champion, M. Gecevičius, M. Beresna, Y. Bellouard, and P. G. Kazansky, "Femtosecond versus picosecond laser machining of nano-gratings and micro-channels in silica glass," Optics Express, vol. 21, no. 4, pp. 3946-3958, Feb. 2013.

50. Y. Shimotsuma, P. G. Kazansky, J. Qiu, and K. Hirao, "Self-Organized Nanogratings in Glass Irradiated by Ultrashort Light Pulses," Physical Review Letters, vol. 91, no. 24, p. 247405, Dec. 2003.

51. E. S. Lein, M. J. Hawrylycz, N. Ao, M. Ayres, A. Bensinger, A. Bernard, A. F. Boe, M. S. Boguski, K. S. Brockway, E. J. Byrnes, L. Chen, L. Chen, T.-M. Chen, M. Chi Chin, J. Chong, B. E. Crook, A. Czaplinska, C. N. Dang, S. Datta, N. R. Dee, A. L. Desaki, T. Desta, E. Diep, T. A. Dolbeare, M. J. Donelan, H.-W. Dong, J. G. Dougherty, B. J. Duncan, A. J. Ebbert, G. Eichele, L. K. Estin, C. Faber, B. A. Facer, R. Fields, S. R. Fischer, T. P. Fliss, C. Frensley, S. N. Gates, K. J. Glattfelder, K. R. Halverson, M. R. Hart, J. G. Hohmann, M. P. Howell, D. P. Jeung, R. A. Johnson, P. T. Karr, R. Kawal, J. M. Kidney, R. H. Knapik, C. L. Kuan, J. H. Lake, A. R. Laramee, K. D. Larsen, C. Lau, T. A. Lemon, A. J. Liang, Y. Liu, L. T. Luong, J. Michaels, J. J. Morgan, R. J. Morgan, M. T. Mortrud, N. F. Mosqueda, L. L. Ng, R. Ng, G. J. Orta, C. C. Overly, T. H. Pak, S. E. Parry, S. D. Pathak, O. C. Pearson, R. B. Puchalski, Z. L. Riley, H. R. Rockett, S. A. Rowland, J. J. Royall, M. J. Ruiz, N. R. Sarno, K. Schaffnit, N. V. Shapovalova, T. Sivisay, C. R. Slaughterbeck, S. C. Smith, K. A. Smith, B. I. Smith, A. J. Sodt, N. N. Stewart, K.-R. Stumpf, S. M. Sunkin, M. Sutram, A. Tam, C. D. Teemer, C. Thaller, C. L. Thompson, L. R. Varnam, A. Visel, R. M. Whitlock, P. E. Wohnoutka, C. K. Wolkey, V. Y. Wong, M. Wood, M. B. Yaylaoglu, R. C. Young, B. L. Youngstrom, X. Feng Yuan, B. Zhang, T. A. Zwingman, and A. R. Jones, "Genome-wide atlas of gene expression in the adult mouse brain," Nature, vol. 445, no. 7124, pp. 168-176, Jan. 2007.

52. K. L. West, N. D. Kelm, R. P. Carson, D. F. Gochberg, K. C. Ess, and M. D. Does, "Myelin volume fraction imaging with MRI," Neurolmage, vol. 182, pp. 511-521, Nov. 2018.

53. D. Kleinfeld, A. Bharioke, P. Blinder, D. D. Bock, K. L. Briggman, D. B. Chklovskii, W. Denk, M. Helmstaedter, J. P. Kaufhold, W.-C. A. Lee, H. S. Meyer, K. D. Micheva, M. Oberlaender, S. Prohaska, R. C. Reid, S. J. Smith, S. Takemura, P. S. Tsai, and B. Sakmann, "Large-scale automated histology in the pursuit of connectomes," Journal of Neuroscience, vol. 31, no. 45, pp. 16125-16 138, Nov. 2011.

54. K. Zilles, N. Palomero-Gallagher, D. Gräßel, P. Schlömer, M. Cremer, R. Woods, K. Amunts, and M. Axer, "Chapter 18 - High-resolution fiber and fiber tract imaging using polarized light microscopy in the human, monkey, rat, and mouse brain," in Axons and Brain Architecture, K. S. Rockland, Ed. San Diego: Academic Press, Jan. 2016, pp. 369-389.

55. B. de Campos Vidal, M. L. S. Mello, A. C. Caseiro-Filho, and C. Godo, "Anisotropic properties of the myelin sheath," Acta Histochemica, vol. 66, no. 1, pp. 32-39, Jan. 1980.

56. M. Menzel, K. Michielsen, H. De Raedt, J. Reckfort, K. Amunts, and M. Axer, "A Jones matrix formalism for simulating three-dimensional polarized light imaging of brain tissue," Journal of The Royal Society Interface, vol. 12, no. 111, p. 20150734, Oct. 2015.

57. E. Min, M. E. Kandel, C. J. Ko, G. Popescu, W. Jung, and C. Best-Popescu, "Label-free, multi-scale imaging of ex-vivo mouse brain using spatial light interference microscopy," Scientific Reports, vol. 6, no. 1, p. 39667, Dec. 2016

58. J. Kwon, M. Kim, H. Park, B.-M. Kang, Y. Jo, J.-H. Kim, O. James, S.-H. Yun, S.-G. Kim, M. Suh, and M. Choi, "Label-free nanoscale optical metrology on myelinated axons in vivo," Nature Communications, vol. 8, no. 1, p. 1832, Nov. 2017.

59. M. Lee, E. Lee, J. Jung, H. Yu, K. Kim, J. Yoon, S. Lee, Y. Jeong, and Y. Park, "Label-free optical quantification of structural alterations in Alzheimer's disease," Scientific Reports, vol. 6, no. 1, p. 31034, Aug. 2016 
bioRxiv preprint doi: https://doi.org/10.1101/2020.12.15.422951; this version posted December 16, 2020. The copyright holder for this preprint (which was not certified by peer review) is the author/funder, who has granted bioRxiv a license to display the preprint in perpetuity. It is made available under aCC-BY-NC-ND 4.0 International license.

$\mathrm{G}$ Automation for multi-scale imaging and analysis

60. L.-A. Harsan, C. Dávid, M. Reisert, S. Schnell, J. Hennig, D. von Elverfeldt, and J. F. Staiger, "Mapping remodeling of thalamocortical projections in the living reeler mouse brain by diffusion tractography," Proceedings of the National Academy of Sciences, vol. 110, no. 19, pp. E1797-E1806, May 2013.

61. T. Chandler, S. Mehta, H. Shroff, R. Oldenbourg, and P. J. L. Rivière, "Single-fluorophore orientation determination with multiview polarized illumination: Modeling and microscope design," Optics Express, vol. 25, no. 25, pp. 31309-31325, Dec. 2017.

62. T. Chandler, H. Shroff, R. Oldenbourg, and P. La Rivière, "Spatio-angular fluorescence microscopy III. Constrained angular diffusion, polarized excitation, and high-NA imaging," arXiv:2001.09232 [physics, q-bio], Jan. 2020

63. T. Eschenhagen, C. Mummery, and B. C. Knollmann, "Modelling sarcomeric cardiomyopathies in the dish: From human heart samples to iPSC cardiomyocytes," Cardiovascular Research, vol. 105, no. 4, pp. 424-438, Apr. 2015.

64. J. C. del Álamo, D. Lemons, R. Serrano, A. Savchenko, F. Cerignoli, R. Bodmer, and M. Mercola, "High throughput physiological screening of iPSC-derived cardiomyocytes for drug development," Biochimica et Biophysica Acta (BBA) - Molecular Cell Research, vol. 1863, no. 7, Part B, pp. 1717-1727, Jul. 2016.

65. J. F. Aronson, "POLARIZED LIGHT OBSERVATIONS ON STRIATED MUSCLE CONTRACTION IN A MITE," The Journal of Cell Biology, vol. 32 , no. 1, pp. $169-179$, Jan. 1967.

66. J. A. Pérez-Bermejo, S. Kang, S. J. Rockwood, C. R. Simoneau, D. A. Joy, G. N. Ramadoss, A. C. Silva, W. R. Flanigan, H. Li, K. Nakamura, J. D. Whitman, M. Ott, B. R. Conklin, and T. C. McDevitt, "SARS-CoV-2 infection of human iPSC-derived cardiac cells predicts novel cytopathic features in hearts of COVID-19 patients," bioRxiv, p. 2020.08.25.265561, Sep. 2020.

67. Yang Xiulan, Pabon Lil, and Murry Charles E., "Engineering Adolescence," Circulation Research, vol. 114, no. 3, pp. 511-523, Jan. 2014.

68. H. Fujii and T. Asakura, "A contrast variation of image speckle intensity under illumination of partially coherent light," Optics Communications, vol. 12, no. 1, pp. 32-38, Sep. 1974.

69. G. Popescu, T. Ikeda, R. R. Dasari, and M. S. Feld, "Diffraction phase microscopy for quantifying cell structure and dynamics," Optics Letters, vol. 31, no. 6, pp. 775-777, Mar. 2006.

70. D. Kim, S. Lee, M. Lee, J. Oh, S.-A. Yang, and Y. Park, "Holotomography: Refractive index as an intrinsic imaging contrast for 3-D label-free live cell imaging," bioRxiv, p. 106328, Nov. 2018.

71. A. D. Yaghjian, A Direct Approach to the Derivation of Electric Dyadic Green's Functions. Nat. Bur. Stand. (U.S.), Tech. Note $1000,1978$.

72. R. Paknys, "Applied Frequency-Domain Electromagnetics," in Applied Frequency-Domain Electromagnetics. John Wiley \& Sons, Ltd, $2016, \mathrm{pp} .335-354$.

73. Emil Wolf, "Unified theory of coherence and polarization of random electromagnetic beams," Physics Letters A, vol. 312, pp. 263-267, Apr. 2003.

74. O. Korotkova and E. Wolf, "Generalized Stokes parameters of random electromagnetic beams," Optics Letters, vol. 30, no. 2, p. 198, Jan. 2005.

75. Joseph Goodman, Statistical Optics. Wiley, 2015.

76. R. A. Chipman, W. S. T. Lam, and G. Young, Polarized Light and Optical Systems, 1st ed. Boca Raton: CRC Press, Aug. 2018.

77. M. R. Kellman, E. Bostan, N. A. Repina, and L. Waller, "Physics-Based Learned Design: Optimized Coded-Illumination for Quantitative Phase Imaging," IEEE Transactions on Computational Imaging, vol. 5, no. 3, pp. 344-353, Sep. 2019.

78. Y. Fan, J. Sun, Q. Chen, X. Pan, L. Tian, and C. Zuo, "Optimal illumination scheme for isotropic quantitative differential phase contrast microscopy," Photonics Research, vol. 7, no. 8, pp. 890-904, Aug. 2019.

79. A. F. Frangi, W. J. Niessen, K. L. Vincken, and M. A. Viergever, "Multiscale vessel enhancement filtering." Springer-Verlag, 1998, pp. $130-137$.

80. Y. Miyaoka, A. H. Chan, L. M. Judge, J. Yoo, M. Huang, T. D. Nguyen, P. P. Lizarraga, P.-L. So, and B. R. Conklin, "Isolation of single-base genome-edited human iPS cells without antibiotic selection," Nature Methods, vol. 11, no. 3, pp. 291-293, Mar. 2014

81. X. Lian, J. Zhang, S. M. Azarin, K. Zhu, L. B. Hazeltine, X. Bao, C. Hsiao, T. J. Kamp, and S. P. Palecek, "Directed cardiomyocyte differentiation from human pluripotent stem cells by modulating Wnt $\beta$-catenin signaling under fully defined conditions," Nature Protocols, vol. 8, no. 1, pp. 162-175, Jan. 2013.

82. S. Tohyama, F. Hattori, M. Sano, T. Hishiki, Y. Nagahata, T. Matsuura, H. Hashimoto, T. Suzuki, H. Yamashita, Y. Satoh, T. Egashira, T. Seki, N. Muraoka, H. Yamakawa, Y. Ohgino, T. Tanaka, M. Yoichi, S. Yuasa, M. Murata, M. Suematsu, and K. Fukuda, "Distinct Metabolic Flow Enables Large-Scale Purification of Mouse and Human Pluripotent Stem Cell-Derived Cardiomyocytes," Cell Stem Cell, vol. 12, no. 1, pp. 127-137, Jan. 2013.

83. J. A. Kong, "Optics of bianisotropic media*," Journal of the Optical Society of America, vol. 64, no. 10, p. 1304, Oct. 1974

84. U. S. Kamilov, D. Liu, H. Mansour, and P. T. Boufounos, "A Recursive Born Approach to Nonlinear Inverse Scattering," IEEE Signal Processing Letters, vol. 23 , no. 8, pp. 1052-1056, Aug. 2016.

85. H.-Y. Liu, D. Liu, H. Mansour, P. T. Boufounos, L. Waller, and U. S. Kamilov, "SEAGLE: Sparsity-Driven Image Reconstruction Under Multiple Scattering," IEEE Transactions on Computational Imaging, vol. 4, no. 1, pp. 73-86, Mar. 2018.

86. M. H. Jenkins and T. K. Gaylord, "Quantitative phase microscopy via optimized inversion of the phase optical transfer function," Applied Optics, vol. 54, no. 28 , p. 8566, Oct. 2015.

87. R. A. Claus, P. P. Naulleau, A. R. Neureuther, and L. Waller, "Quantitative phase retrieval with arbitrary pupil and illumination," Optics Express, vol. 23 , no. 20 , pp. 26 672-26 682, Oct. 2015.

88. M. R. Foreman and P. Török, "Computational methods in vectorial imaging," Journal of Modern Optics, vol. 58, no. 5-6, pp. 339-364, Mar. 2011.

89. J. R. Fienup, "Phase retrieval algorithms: A comparison," Applied Optics, vol. 21, no. 15, pp. 2758-2769, Aug. 1982.

90. L.-H. Yeh, J. Dong, J. Zhong, L. Tian, M. Chen, G. Tang, M. Soltanolkotabi, and L. Waller, "Experimental robustness of Fourier ptychography phase retrieval algorithms," Optics Express, vol. 23 , no. 26, pp. 33214-33240, Dec. 2015 
bioRxiv preprint doi: https://doi.org/10.1101/2020.12.15.422951; this version posted December 16,2020 . The copyright holder for this preprint

(which was not certified by peer review) is the author/funder, who has granted bioRxiv a license to display the preprint in perpetuity. It is made available under aCC-BY-NC-ND 4.0 International license.

\section{Supplementary Figures}

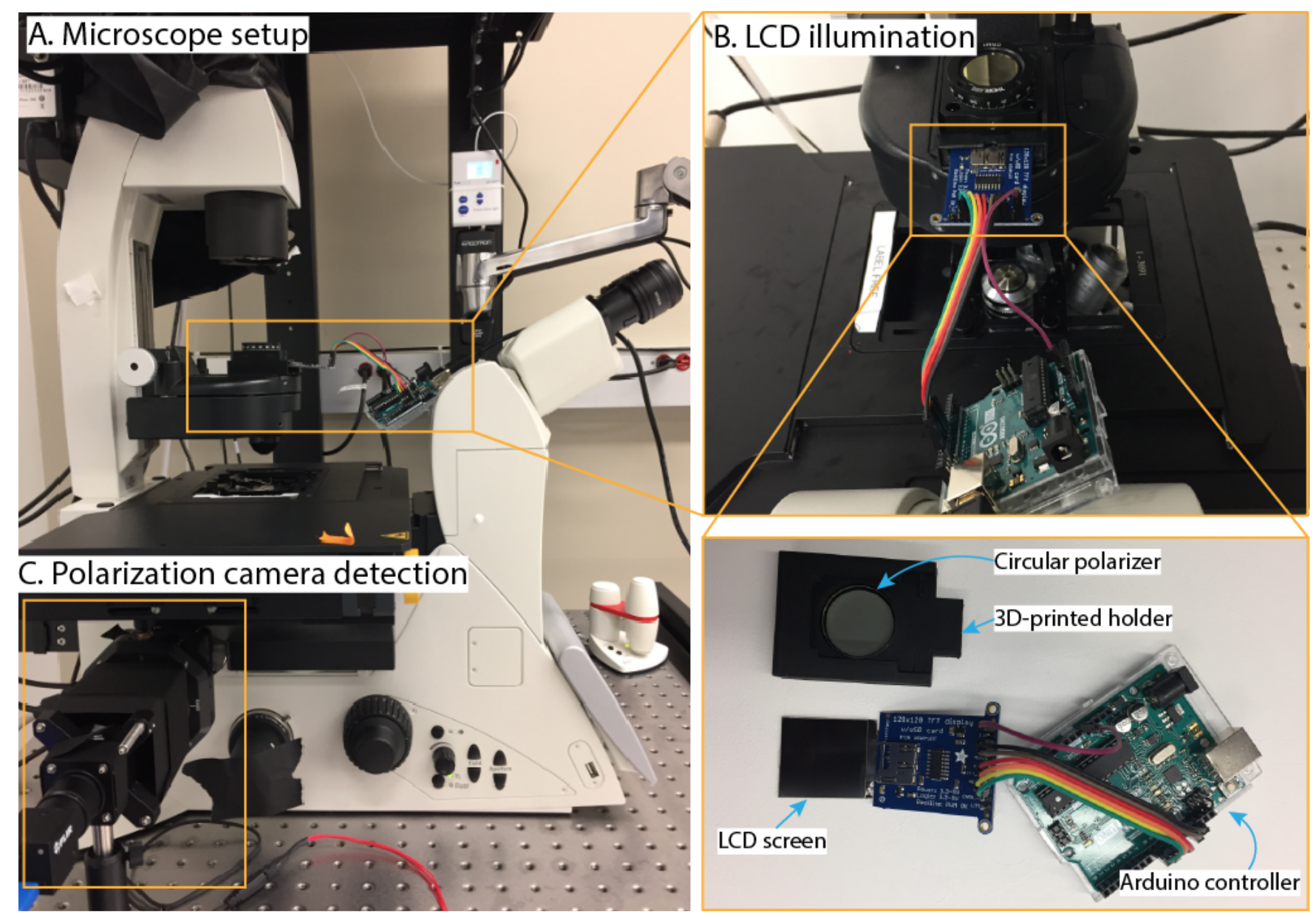

Figure 1-supplement 1. Photographs of the uPTI setup: (A) The microscope setup. (B) The add-on LCD illumination setup composed of adafruit LCD screen (Adafruit, ST7735R) and the circular polarizer (Thorlabs, CP1R532). (C) The add-on polarization camera (FLIR, BFS-U3-51S5P-C) for detection. 
bioRxiv preprint doi: https://doi.org/10.1101/2020.12.15.422951; this version posted December 16,2020 . The copyright holder for this preprint (which was not certified by peer review) is the author/funder, who has granted bioRxiv a license to display the preprint in perpetuity. It is made available under aCC-BY-NC-ND 4.0 International license.

$\mathrm{G}$ Automation for multi-scale imaging and analysis

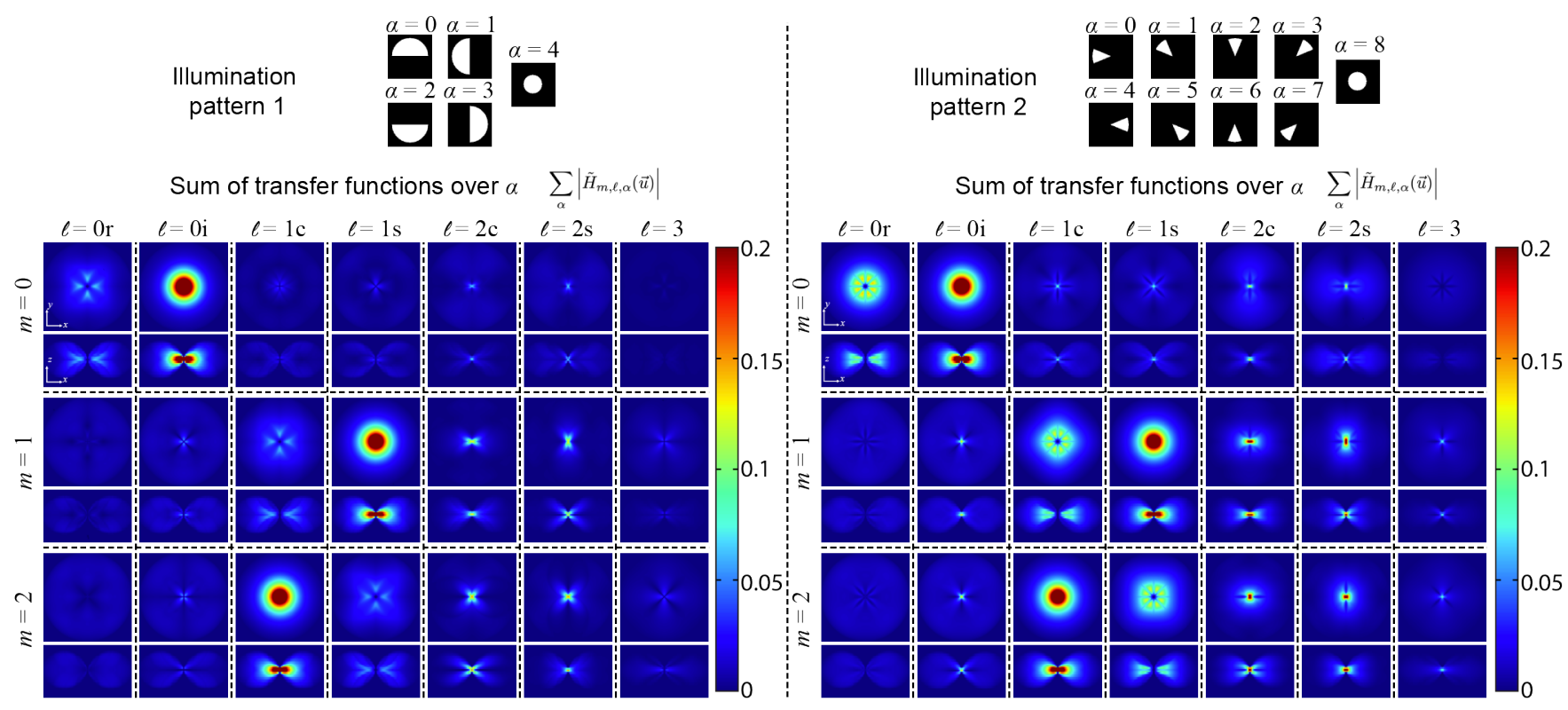

Figure 1-supplement 2. Comparison of transfer functions for two sets of illumination patterns: Sum of transfer functions (mapping from the $\ell$-th individual scattering potential tensor component to the $m$-th Stokes parameres) over all illumination patterns $(\alpha)$ with the combination of semi-circular (typically used for differential phase contrast) and brightfield illumination (left) and the combination of sector and brightfield illumination (right). We adopt the right set of illumination pattern, which transfers more uniformly across 3D spatial frequencies in all tensor components. 
bioRxiv preprint doi: https://doi.org/10.1101/2020.12.15.422951; this version posted December 16, 2020. The copyright holder for this preprint (which was not certified by peer review) is the author/funder, who has granted bioRxiv a license to display the preprint in perpetuity. It is made available under aCC-BY-NC-ND 4.0 International license.

3D orientation of laser-written birefringent target

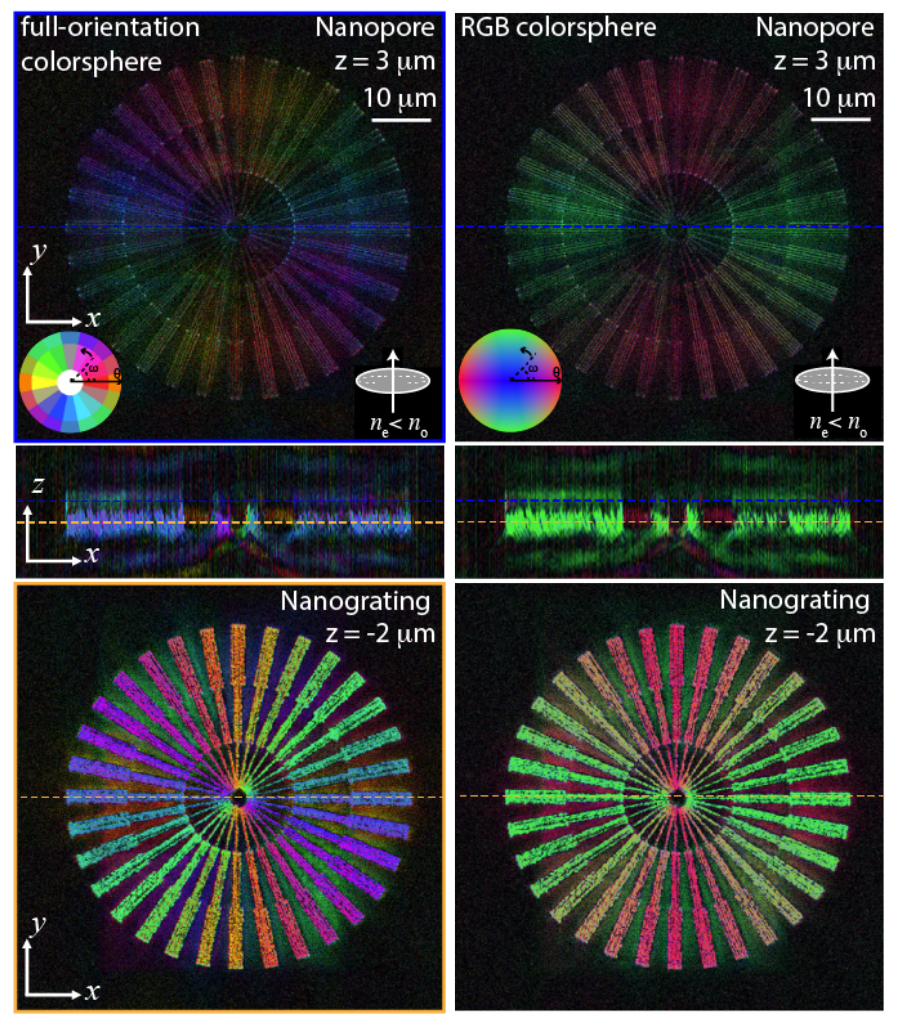

Figure 2-supplement 1. 3D orientation of the anisotropic glass modified with femtosecond laser: The 3D orientation of the anisotropic glass shown in fig. $2 \mathrm{C}$ is rendered with the full-orientation colorsphere (left) and the RGB colorsphere (right). The full-orientation colorsphere encodes full range (upper hemisphere) of measurable $3 \mathrm{D}$ orientation, but is more complex to read. The RGB colorsphere maps the absolute $x, y$, and $z$ components of the 3D orientation into red, green, and blue color, which is more intuitive to read, but maps only one octant of the $3 \mathrm{D}$ orientation, i.e., quarter of the measurable range. From both renderings, we see the $3 \mathrm{D}$ orientation of the target is perpendicular to the spoke feature and mostly in the $x-y$ plane. 
bioRxiv preprint doi: https://doi.org/10.1101/2020.12.15.422951; this version posted December 16,2020 . The copyright holder for this preprint (which was not certified by peer review) is the author/funder, who has granted bioRxiv a license to display the preprint in perpetuity. It is made available under aCC-BY-NC-ND 4.0 International license.

$\mathrm{G}$ Automation for multi-scale imaging and analysis

\section{Laser written birefringent target with different writing parameters (double line-scan, 300 -fs pulse duration, nanograting)}
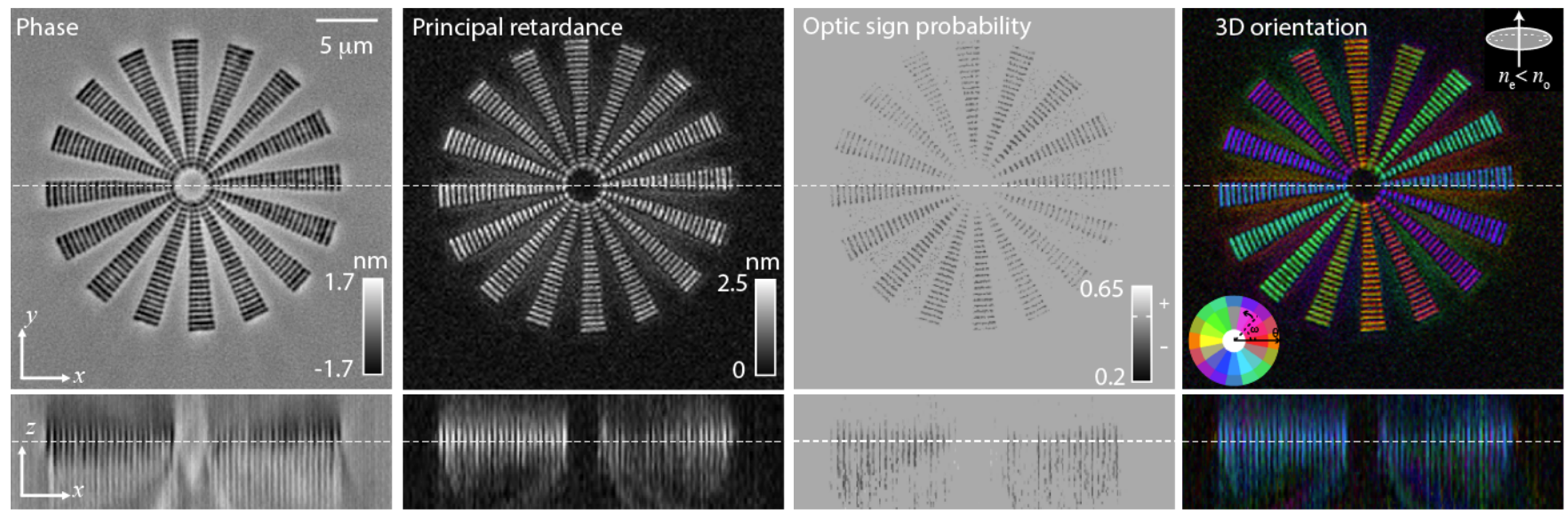

Figure 2-supplement 2. uPTI discriminates the properties of laser written anisotropic glass as writing parameters are tuned: Phase, principal retardance, optic sign probability and 3D orientation of a nanograting birefringent target that was written with 300 -fs laser pulse duration, $515 \mathrm{~nm}$ wavelength, and illumination NA of 0.55 using a double line scan process, which writes a feature by scanning the laser two times in opposite directions. Differences in the laser parameters relative to the target shown in fig. 2 prevents the creation of two layers of modification as shown in fig. $2 \mathrm{C}$. In addition, the double line scan creates more uniform line features compared to fig. $2 \mathrm{C}$, where we see stronger phase and principal retardance at the end of each grating line within a spoke. 
bioRxiv preprint doi: https://doi.org/10.1101/2020.12.15.422951; this version posted December 16, 2020. The copyright holder for this preprint (which was not certified by peer review) is the author/funder, who has granted bioRxiv a license to display the preprint in perpetuity. It is made available under aCC-BY-NC-ND 4.0 International license.
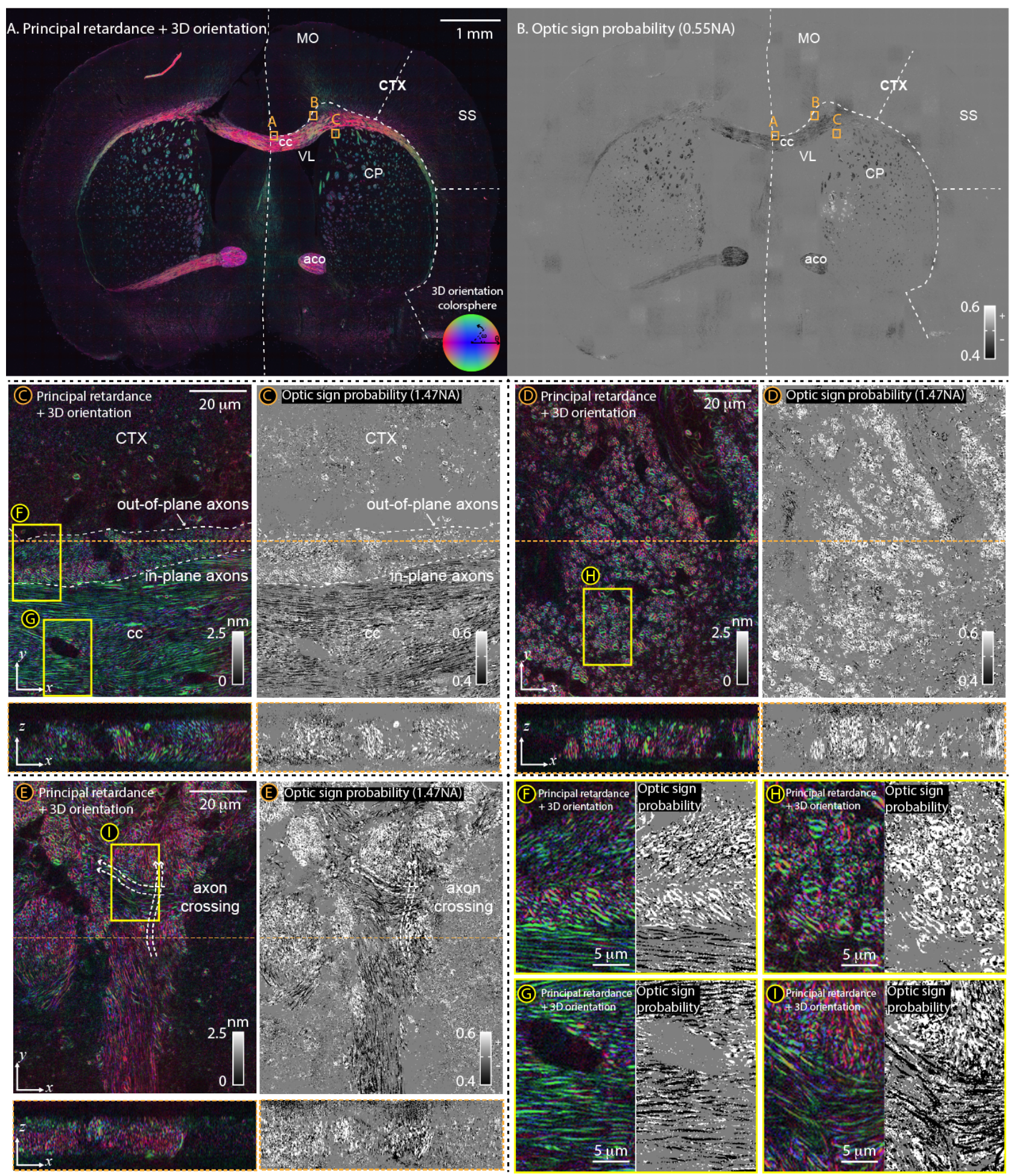

Figure 3-supplement 1. 3D orientation with RGB colorsphere and optic sign probability of an adult mouse brain section: (top row) $2 \mathrm{D}$ low-resolution imaging of the whole brain section with (A) principal retardance, 3D orientation and (B) optic sign probability channels. (bottom: C, D, and E) 3D high-resolution principal retardance, 3D orientation with RGB colorsphere and optic sign probability of three areas marked with orange boxes in top row. At the imaging and illumination NA of 0.55 (spatial resolution of $\sim 0.5 \times 0.5 \times 3.2 \mu \mathrm{m}$ ), axons behave like negative uniaxial material as seen in the optic sign probability. Therefore, we assume the negative uniaxial material when displaying 3D orientation over the whole slide. Multiple anatomical landmarks are labeled according to the coronal section at level 51 of the Allen brain reference atlas (51), aco: anterior commissure (olfactory limb), cc: corpus callosum, CP: caudoputamen, CTX: cortex, MO: motor cortex, SS: somatosensory area, VL: ventricle. Following areas are imaged in high-resolution 3D mode with imaging NA of 1.47 and illumination NA of 1.4 (spatial resolution of $\sim 0.25 \times 0.25 \times 0.8 \mu \mathrm{m}$ ): (C) the boundary of the corpus callosum and the cortex region, (D) the cingulum bundle region, and (E) the fiber crossing in the caudoputamen region. We further zoom in four parts from (C-E) to demonstrate complex axon networks in (F-I). With high resolution, we resolve the boundaries of individual axons, which behave as the positive uniaxial material with 3D orientation perpendicular to the surface of the membrane. The RGB colorsphere provides an easier interpretation to see if the structure is (red and green) or isn't (blue) in the $x-y$ plane. 
bioRxiv preprint doi: https://doi.org/10.1101/2020.12.15.422951; this version posted December 16, 2020. The copyright holder for this preprint (which was not certified by peer review) is the author/funder, who has granted bioRxiv a license to display the preprint in perpetuity. It is made available under aCC-BY-NC-ND 4.0 International license.

$\mathrm{G}$ Automation for multi-scale imaging and analysis

\section{Permittivity tensor at low-resolution (assume negative uniaxial): 3D orientation of axons}
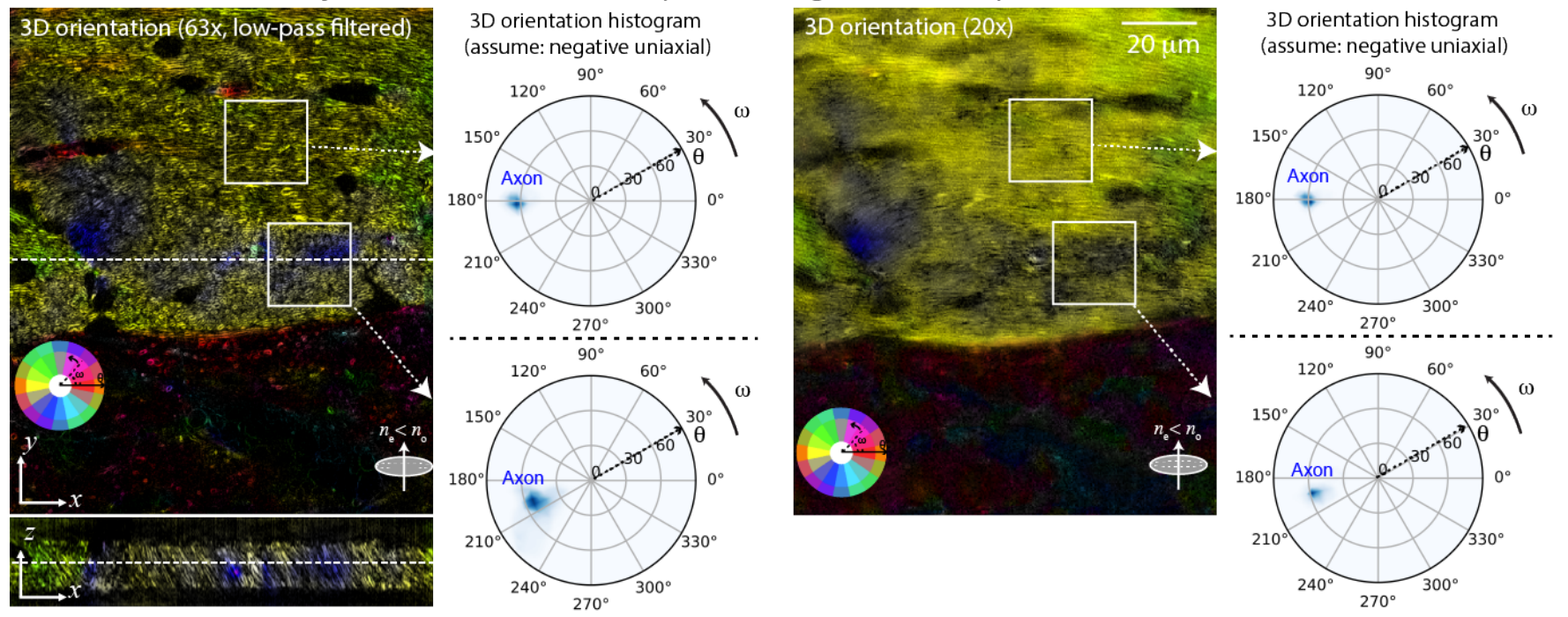

Figure 3-supplement 2. Interpretation of 3D orientation measurements at low resolution: The 3D orientation measurements of two axon bundles (the same region as shown in fig. 4) computed from (left) the low-pass filtered scattering potential tensor acquired at $63 \times$ objective (1.47 NA) and (right) the scattering potential tensor acquired at $20 \times$ objective $(0.55 \mathrm{NA})$ assuming the specimen is negative uniaxial. The averaged orientation measurements acquired at $63 \times$ show similar distribution of $3 \mathrm{D}$ orientation to the distribution measured at $20 \times$, indicating that uPTI provides sensitive measurement of $3 \mathrm{D}$ anisotropy across different spatial scales. 
bioRxiv preprint doi: https://doi.org/10.1101/2020.12.15.422951; this version posted December 16,2020 . The copyright holder for this preprint (which was not certified by peer review) is the author/funder, who has granted bioRxiv a license to display the preprint in perpetuity. It is made available under aCC-BY-NC-ND 4.0 International license.

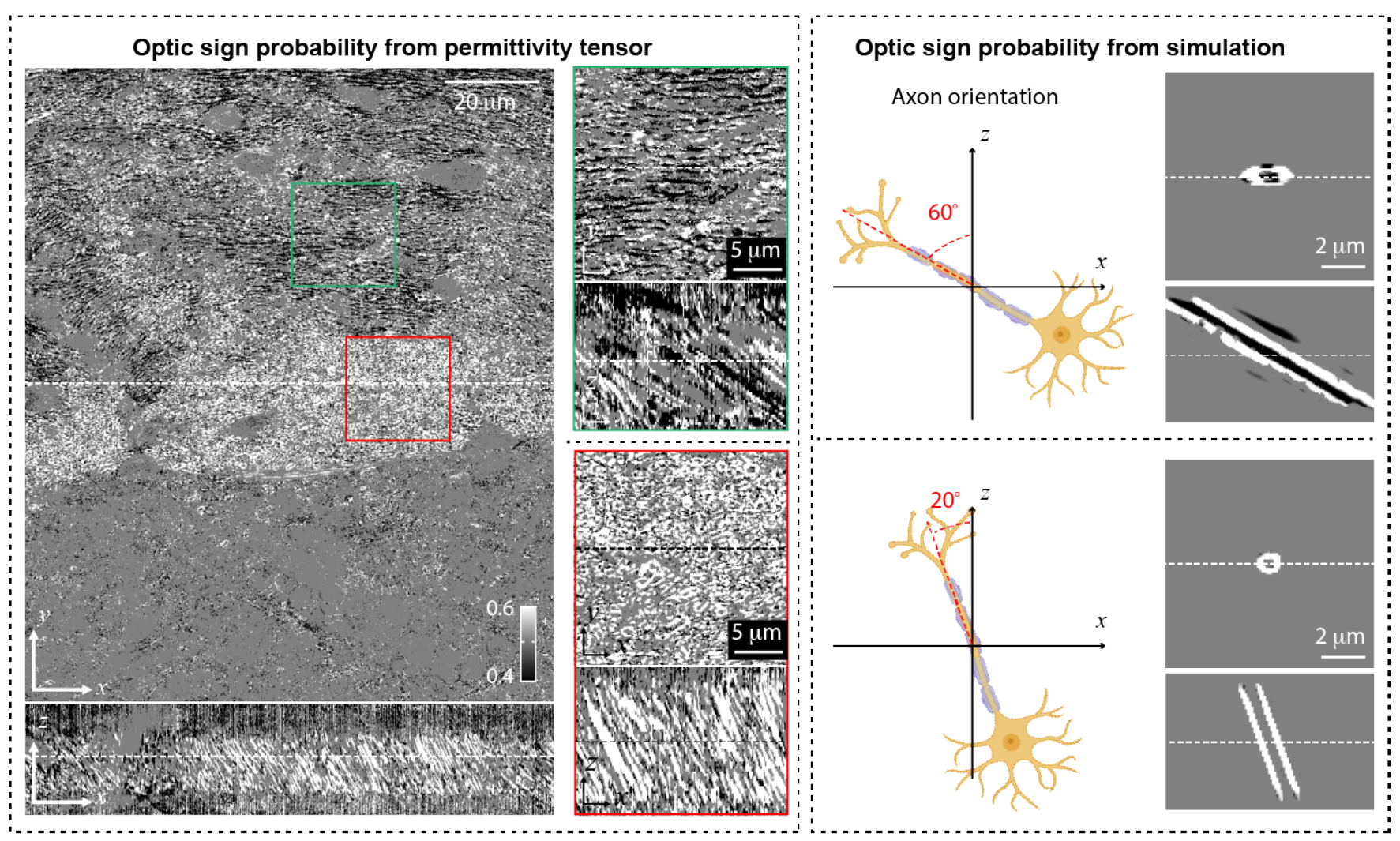

Figure 4-supplement 1. Interpreting the dependence of the optic sign of axons on their inclination with a simulation: We compare high-resolution maps of the optic sign probability in the volume of mouse brain tissue shown in fig. 4 against the optic sign probability of a single simulated axon. The data showed that majority of axons are inclined at $60^{\circ}$ and at $20^{\circ}$ in the green and red FOVs. We notice that as the axon inclination changes from the microscope axis $(z)$ to the focal plane $(x-y)$, the optic sign of the voxels changes from positive to negative. We interpret these variation by simulating permittivity tensor of single axons (illustrated by myelinated neuron in the cartoon) inclined at $60^{\circ}$ and $20^{\circ}$. The experimental results match well with the simulated results in these two axon inclinations: The optic sign is mostly positive on voxels that span the axon cross sections when axon is inclined at $20^{\circ}$ and it oscillates between positive and negative when axon is inclined at $60^{\circ}$. 
bioRxiv preprint doi: https://doi.org/10.1101/2020.12.15.422951; this version posted December 16,2020 . The copyright holder for this preprint (which was not certified by peer review) is the author/funder, who has granted bioRxiv a license to display the preprint in perpetuity. It is made available under aCC-BY-NC-ND 4.0 International license.

$\mathrm{G}$ Automation for multi-scale imaging and analysis

\section{Structure tensor: 3D orientation from phase}

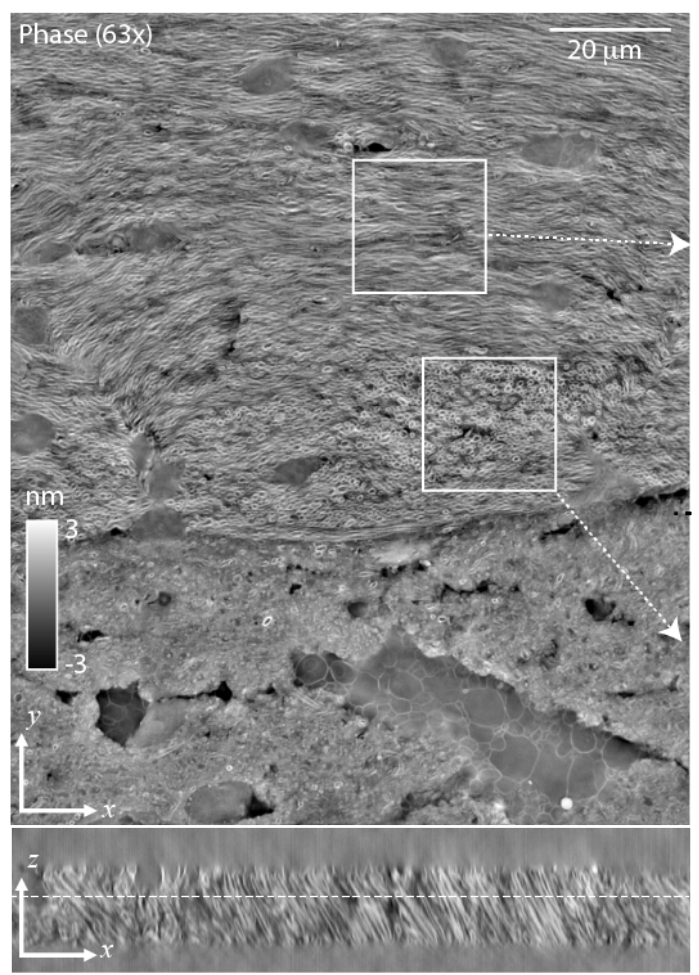

3D orientation histogram

( $1^{\text {st }}$ eigenvector, lipid orientation)

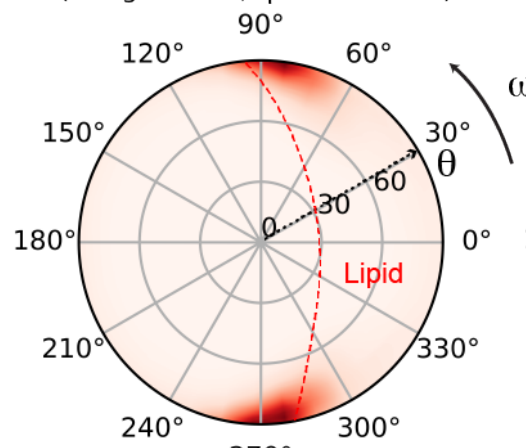
$270^{\circ}$

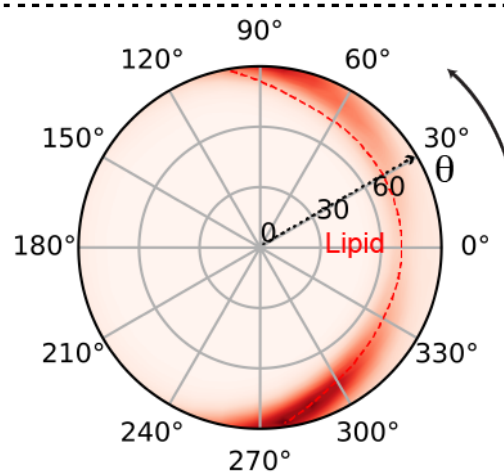

3D orientation histogram

( $3^{\text {rd }}$ eigenvector, axon orientation)

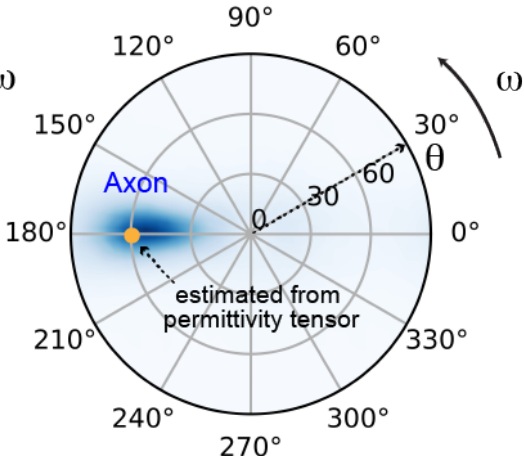

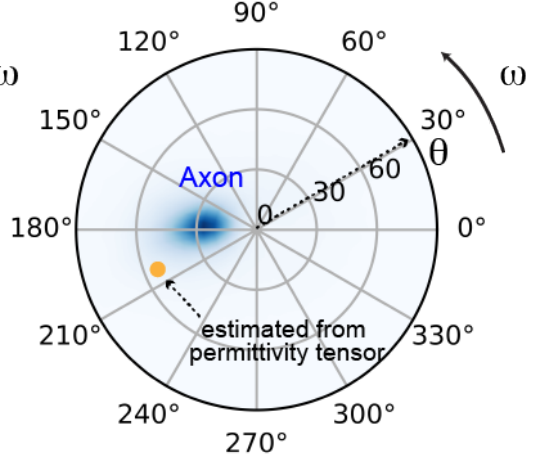

Figure 4-supplement 2. Comparison of 3D orientation of the permittivity tensors and the structure tensors at high resolution: The 3D orientation of the largest (orientation of lipids) and the smallest (orientation of axons) eigenvector of the structure tensor of the phase image for two same regions. The 3D orientation of the third eigenvector of the structure tensor matches with the 3D orientation of axons estimated from the low-resolution permittivity tensor with the assumption of negative uniaxial material (indicated by an orange dot). Slight bias of the 3D orientation from permittivity tensor is observed when axons are inclined toward the imaging axis. Similarly, when the boundaries of axons are resolved, the 3D orientation from the high-resolution permittivity tensor with the assumption of positive uniaxial material matches with the 3D orientation of the first eigenvector of the structure tensor. 
bioRxiv preprint doi: https://doi.org/10.1101/2020.12.15.422951; this version posted December 16, 2020. The copyright holder for this preprint

(which was not certified by peer review) is the author/funder, who has granted bioRxiv a license to display the preprint in perpetuity. It is made available under aCC-BY-NC-ND 4.0 International license.

Simulated permittivity tensor as a function of axon orientation
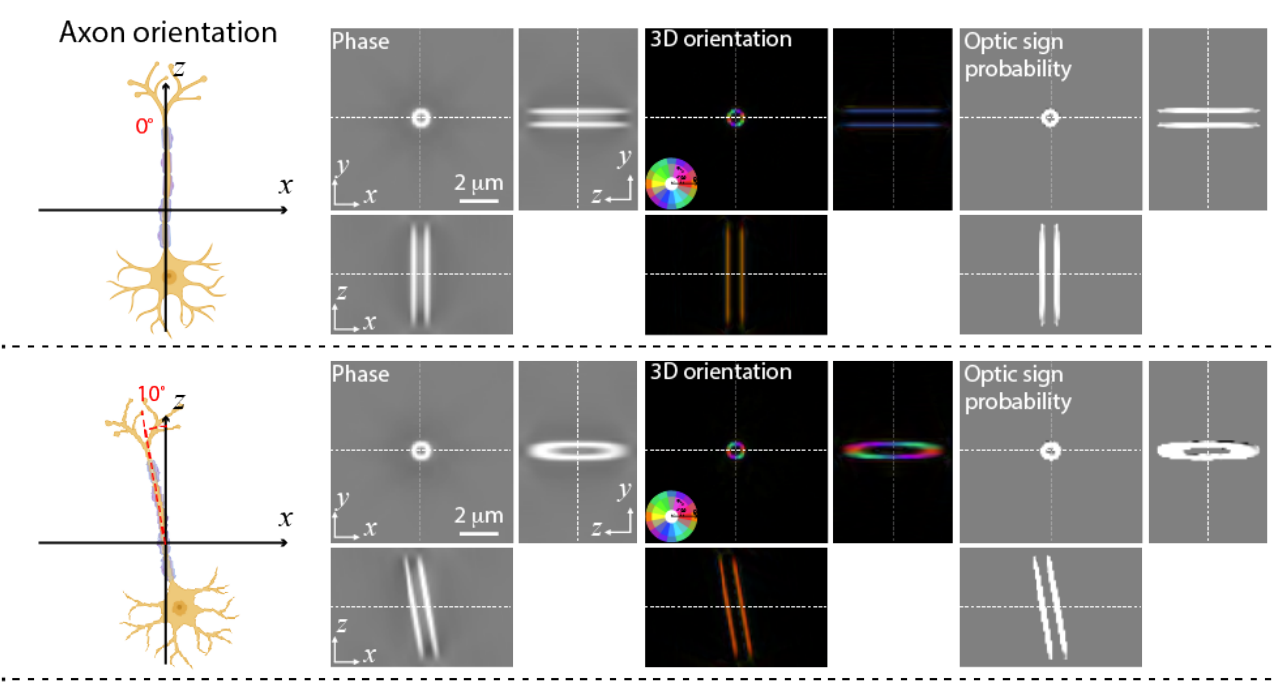

3D orientation histogram
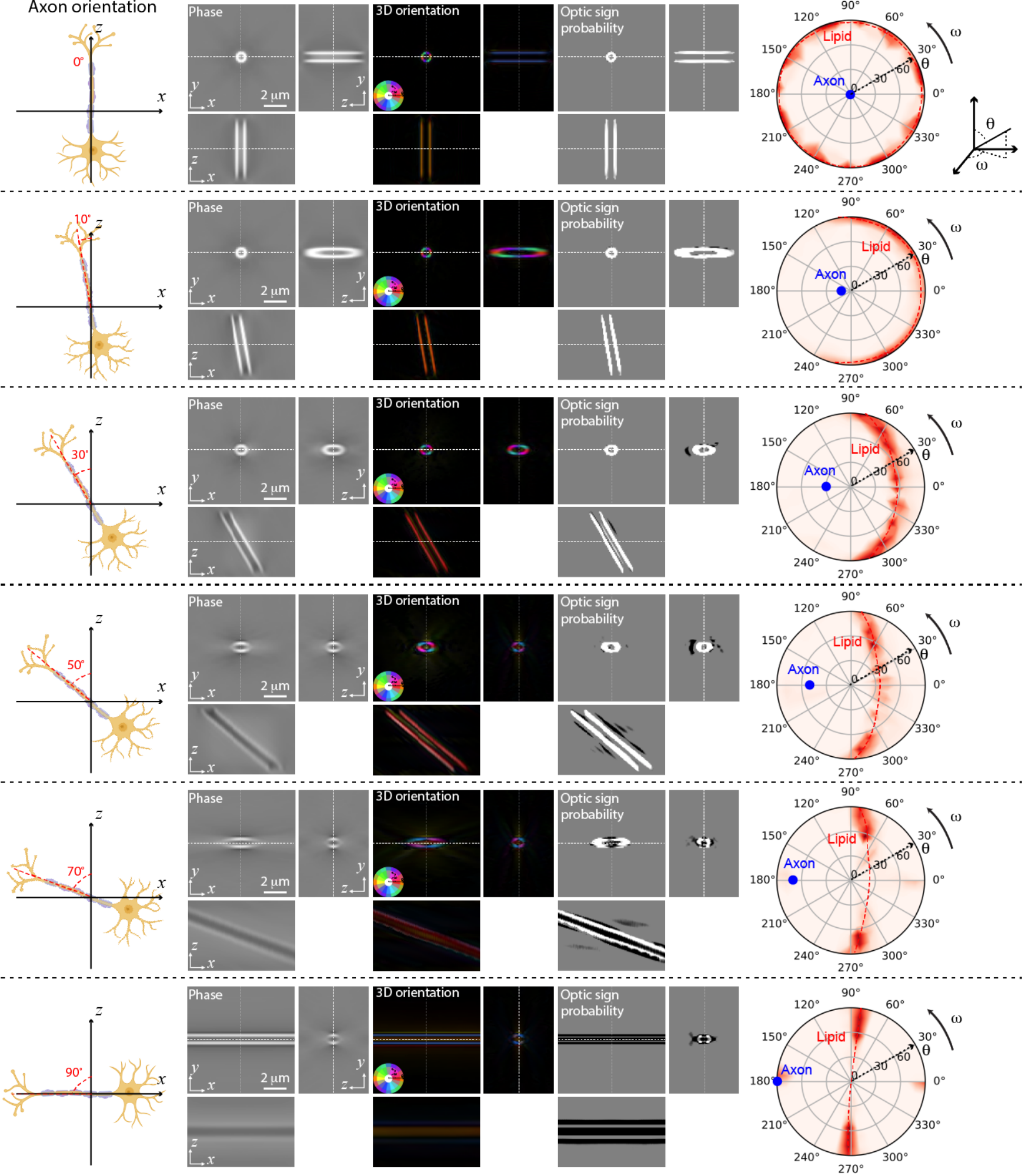

Figure 4-supplement 3. Sensitivity of measured uniaxial permittivity tensor to inclination of anisotropic material: Simulated reconstruction of the uniaxial permittivity tensor components for single axons tilted with $0^{\circ}, 10^{\circ}, 30^{\circ}, 50^{\circ}, 70^{\circ}, 90^{\circ}$ from $z$-axis to the left. In this simulation, we assume an axon is a perfect cylindrical shell composed of uniform positive uniaxial material with radially arranged 3D orientation around the cylinder axis. The reconstruction (phase, principal retardance, 3D orientation and optic sign) generally performs better when axons incline slightly from $z$-axis, corresponding to the case when the 3D orientation of lipid molecules in axon cross sections lies within the $x-y$ plane. The histograms of 3D orientation of the axon cross sections shows a circular shape when the axon is oriented along $z$. The 3D orientation of axon cross sections shifts gradually towards the pole of the top hemisphere as the axon inclines towards the $x-y$ plane. The 3D distribution gets distorted when axon inclination is larger than about $70^{\circ}$ or when inclination of axon cross sections is less than $20^{\circ}$, because of poorer sensitivity of our method to the anisotropy aligned with the microscope axis. 
bioRxiv preprint doi: https://doi.org/10.1101/2020.12.15.422951; this version posted December 16,2020 . The copyright holder for this preprint (which was not certified by peer review) is the author/funder, who has granted bioRxiv a license to display the preprint in perpetuity. It is made available under aCC-BY-NC-ND 4.0 International license.

$\mathrm{G}$ Automation for multi-scale imaging and analysis

\section{Imaging of fixed cardiomyocytes}
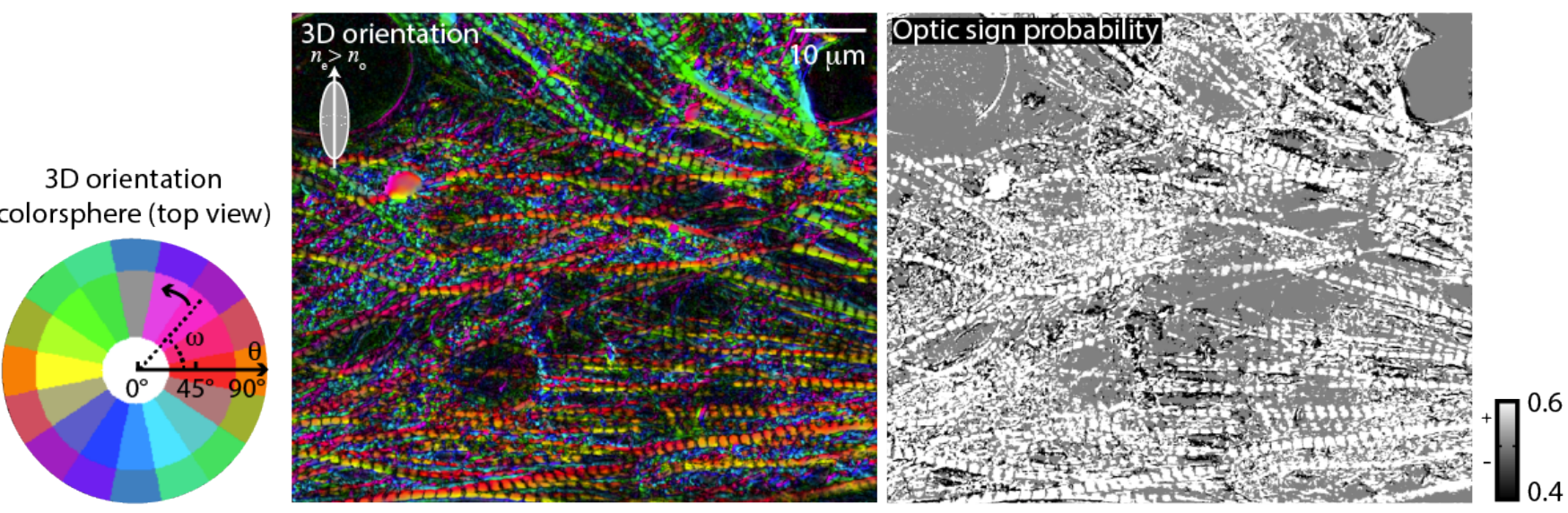

Figure 5-supplement 1. 3D orientation and optic sign probability of the iPSC-derived cardiomyocytes: The 3D orientation and optic sign probability of the (A) healthy and (B) SARS-COV-2 infected cardiomyocytes shown in fig. 5 . In the healthy cardiomyocytes, the optic sign of the sarcomere structure is found to be a positive and $3 \mathrm{D}$ orientation is found to point along the axis of the sarcomere. In the infected cardiomyocytes, the principal retardance drops as seen in fig. $5 \mathrm{D}$, which makes the optic sign estimate noisier. The 3D orientation map in the infected cells shows small stretches of consistent orientation that suggests broken sarcomeres, but the orientation estimate is also noisier due to reduced retardance. 
bioRxiv preprint doi: https://doi.org/10.1101/2020.12.15.422951; this version posted December 16, 2020. The copyright holder for this preprint (which was not certified by peer review) is the author/funder, who has granted bioRxiv a license to display the preprint in perpetuity. It is made available under aCC-BY-NC-ND 4.0 International license.

\section{Video}

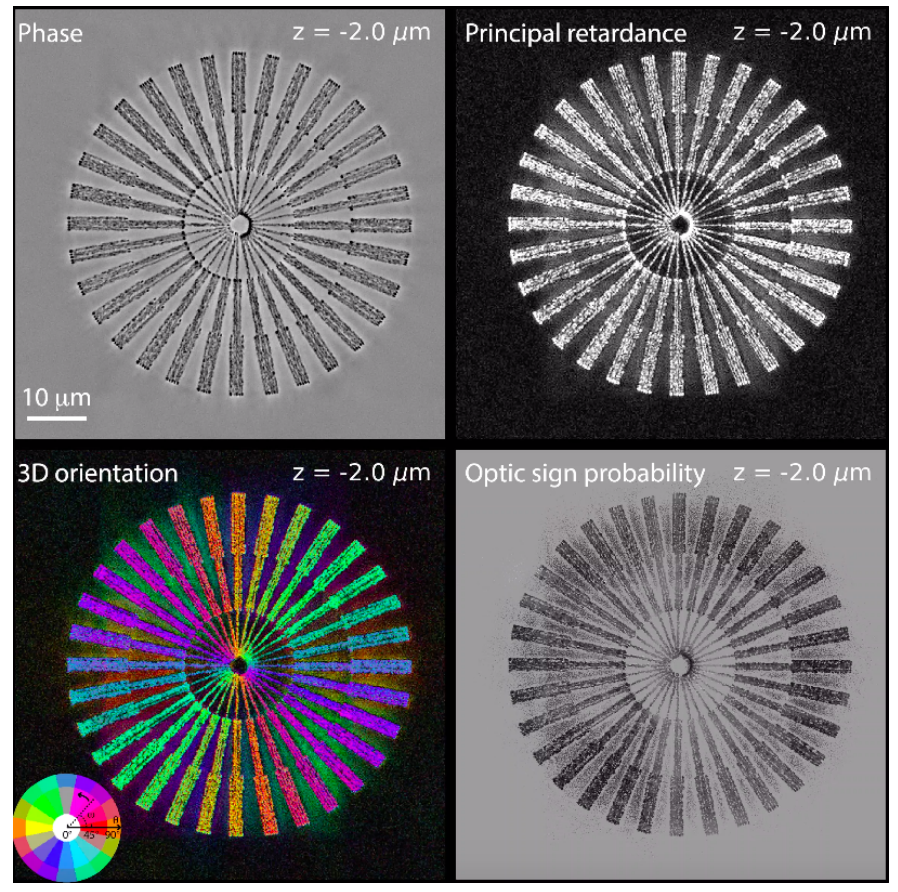

Video 1. $z$-stacks of phase, principal retardance, 3D orientation and optic sign probability images of the laser written birefringent target shown in fig. 2.

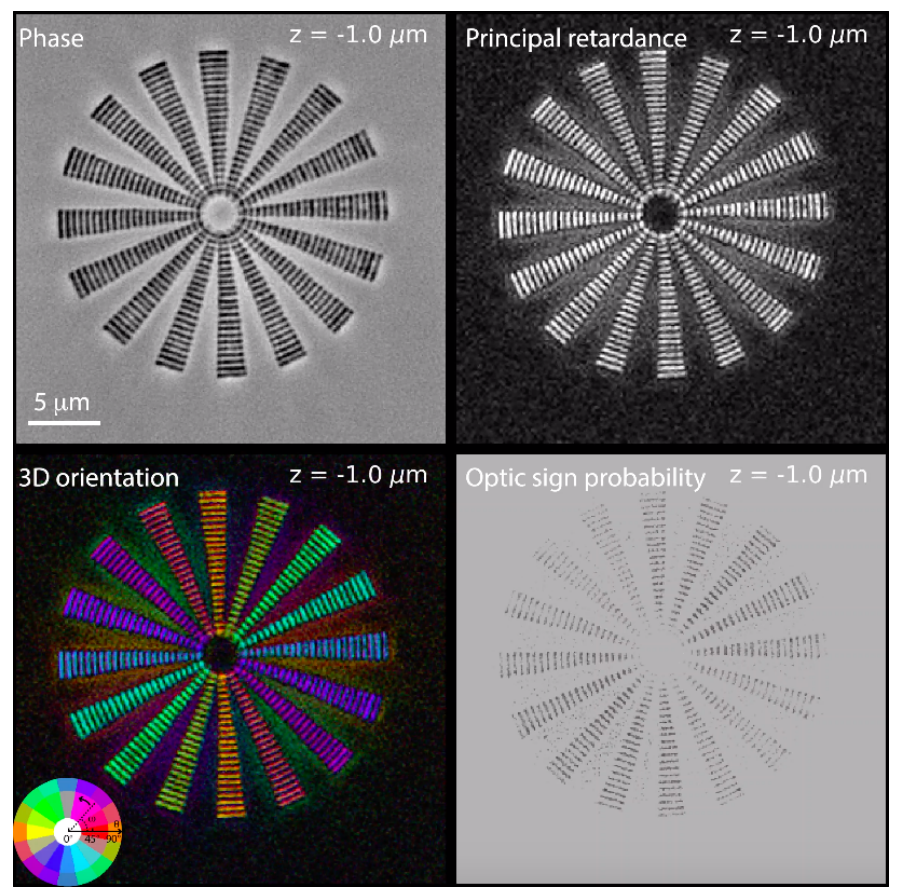

Video 2. $z$-stacks of phase, principal retardance, 3D orientation and optic sign probability images of the laser written birefringent target shown in Figure 2-supplement 2. 
bioRxiv preprint doi: https://doi.org/10.1101/2020.12.15.422951; this version posted December 16, 2020. The copyright holder for this preprint

(which was not certified by peer review) is the author/funder, who has granted bioRxiv a license to display the preprint in perpetuity. It is made available under aCC-BY-NC-ND 4.0 International license.

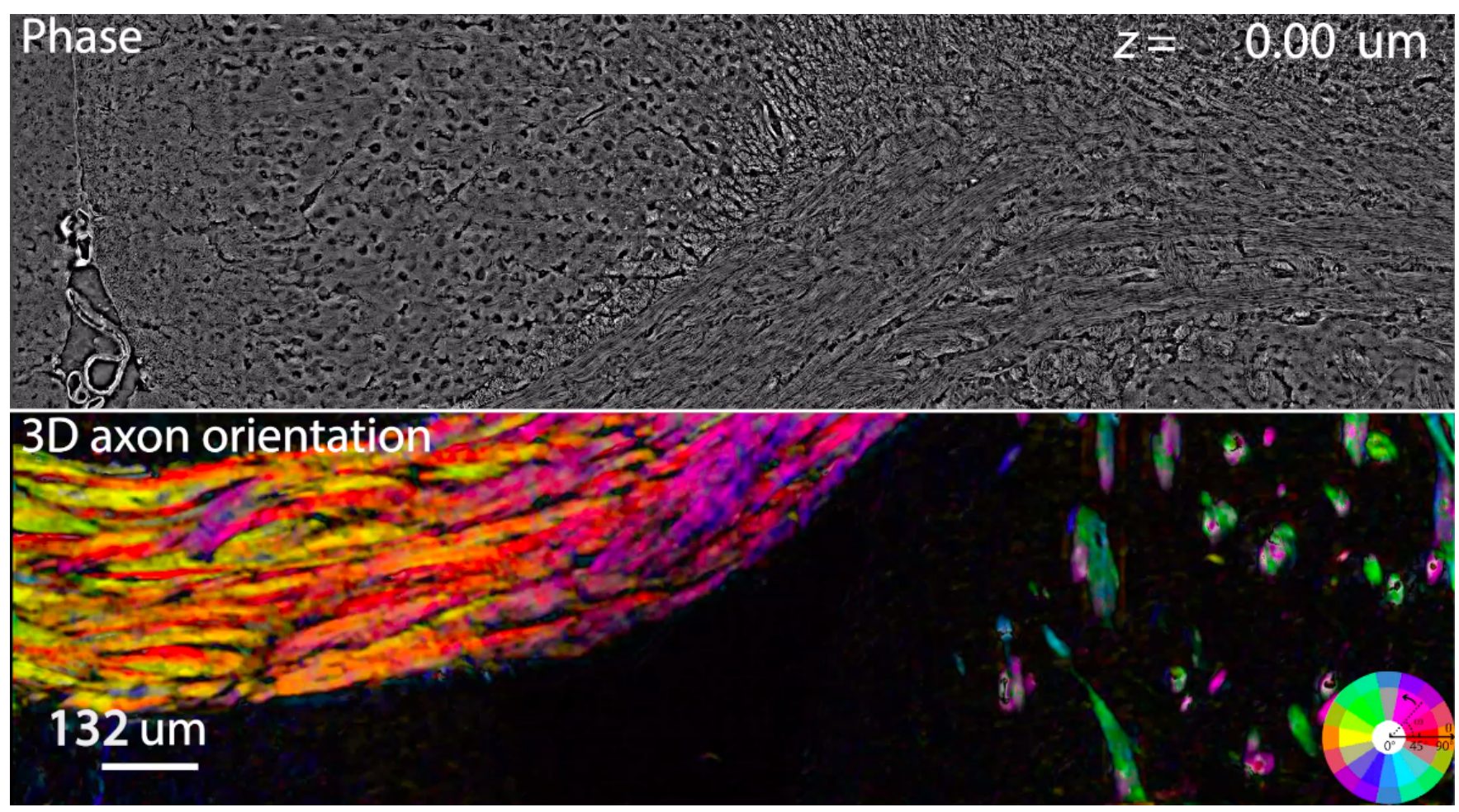

Video 3. Visualization of 3D high-resolution (1.47NA) phase and principal retardance of the adult mouse brain section.

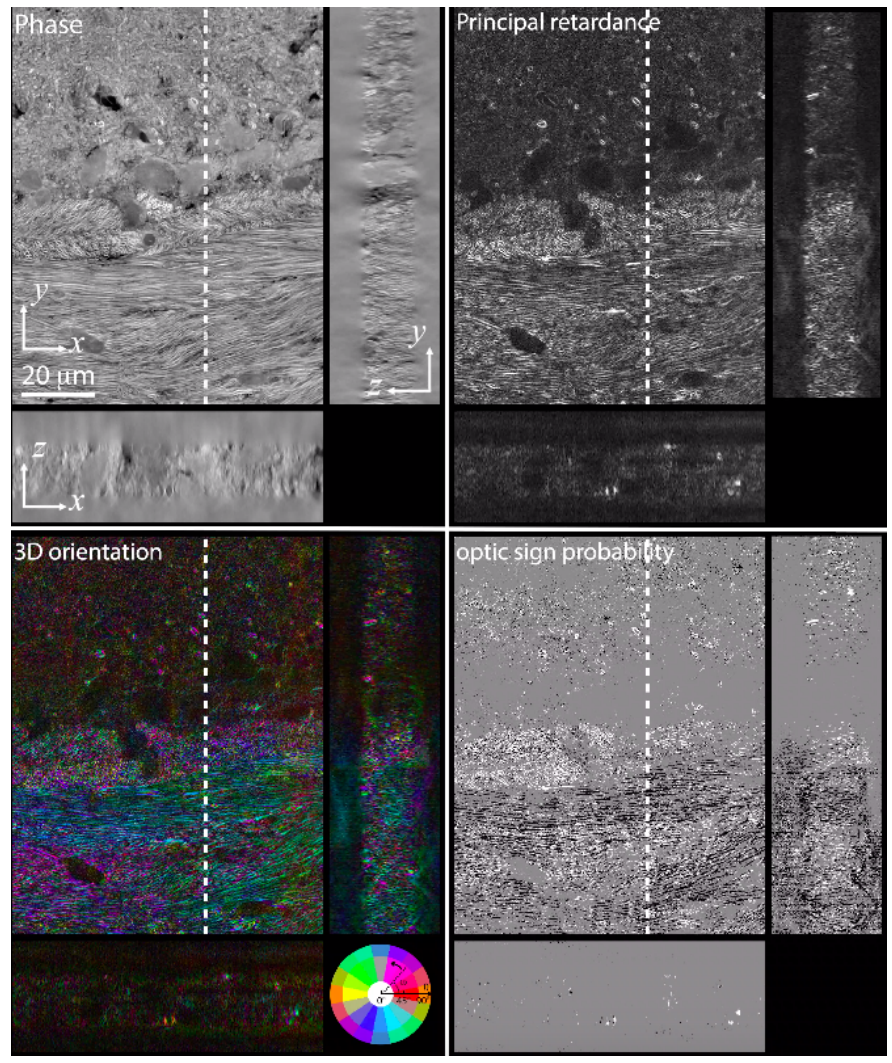

Video 4. $z$-stacks, $x-z$ section, and $y-z$ section of phase, principal retardance, 3D orientation and optic sign probability images of field of view A of the mouse brain section shown in fig. 3. 
bioRxiv preprint doi: https://doi.org/10.1101/2020.12.15.422951; this version posted December 16, 2020. The copyright holder for this preprint (which was not certified by peer review) is the author/funder, who has granted bioRxiv a license to display the preprint in perpetuity. It is made available under aCC-BY-NC-ND 4.0 International license.
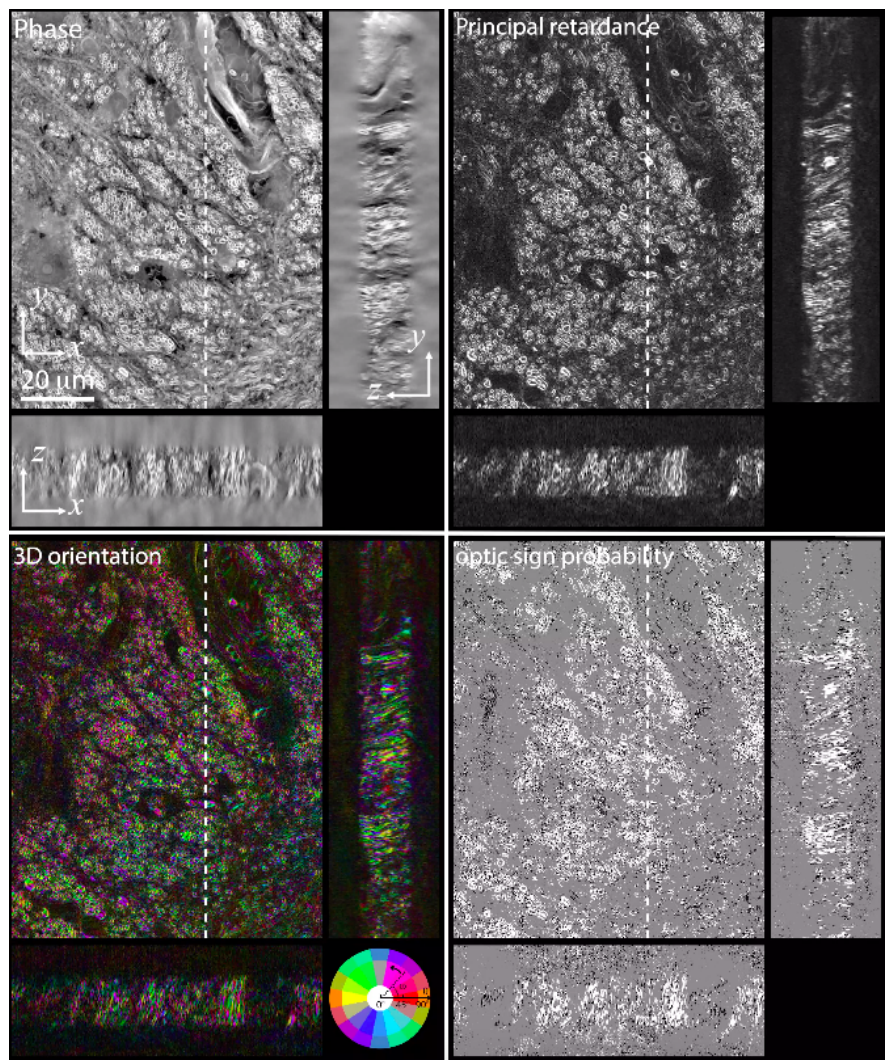

Video 5. $z$-stacks, $x$ - $z$ section, and $y-z$ section of phase, principal retardance, 3D orientation and optic sign probability images of field of view $\mathrm{B}$ of the mouse brain section shown in fig. 3.
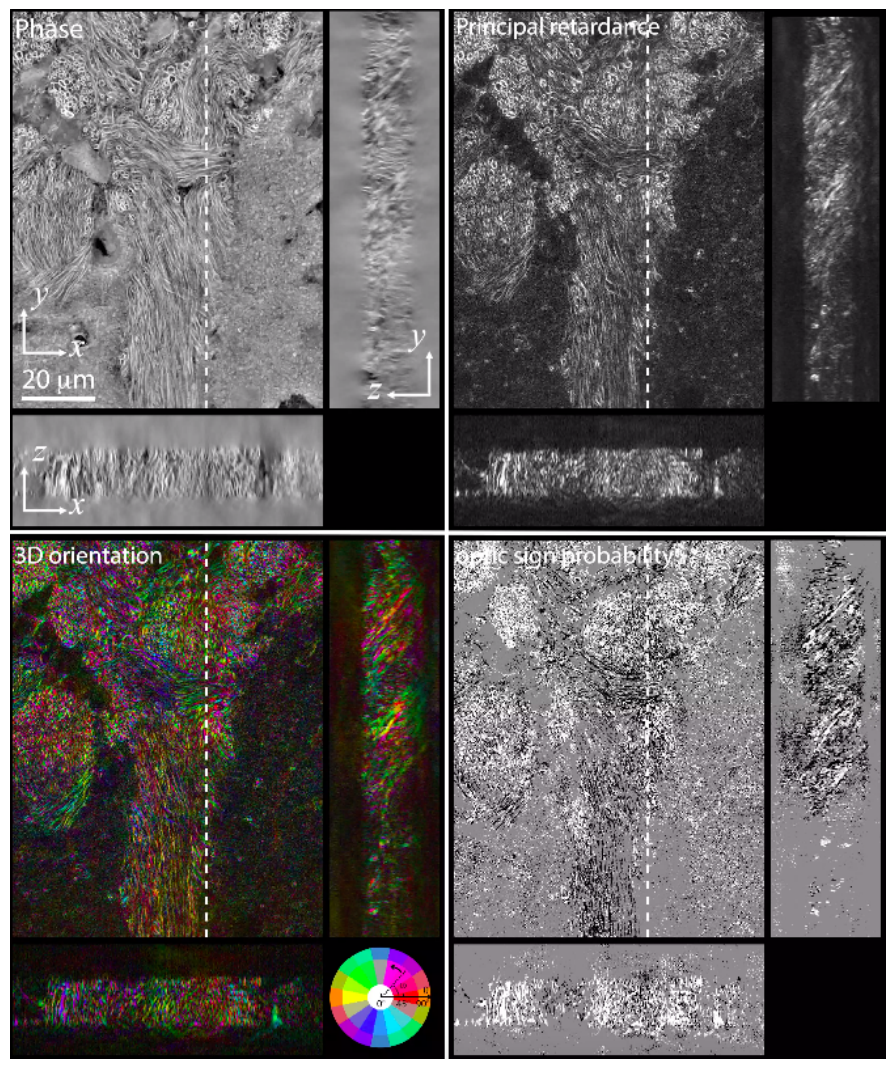

Video 6. $z$-stacks, $x$ - $z$ section, and $y$ - $z$ section of phase, principal retardance, 3D orientation and optic sign probability images of field of view $\mathrm{C}$ of the mouse brain section shown in fig. 3. 

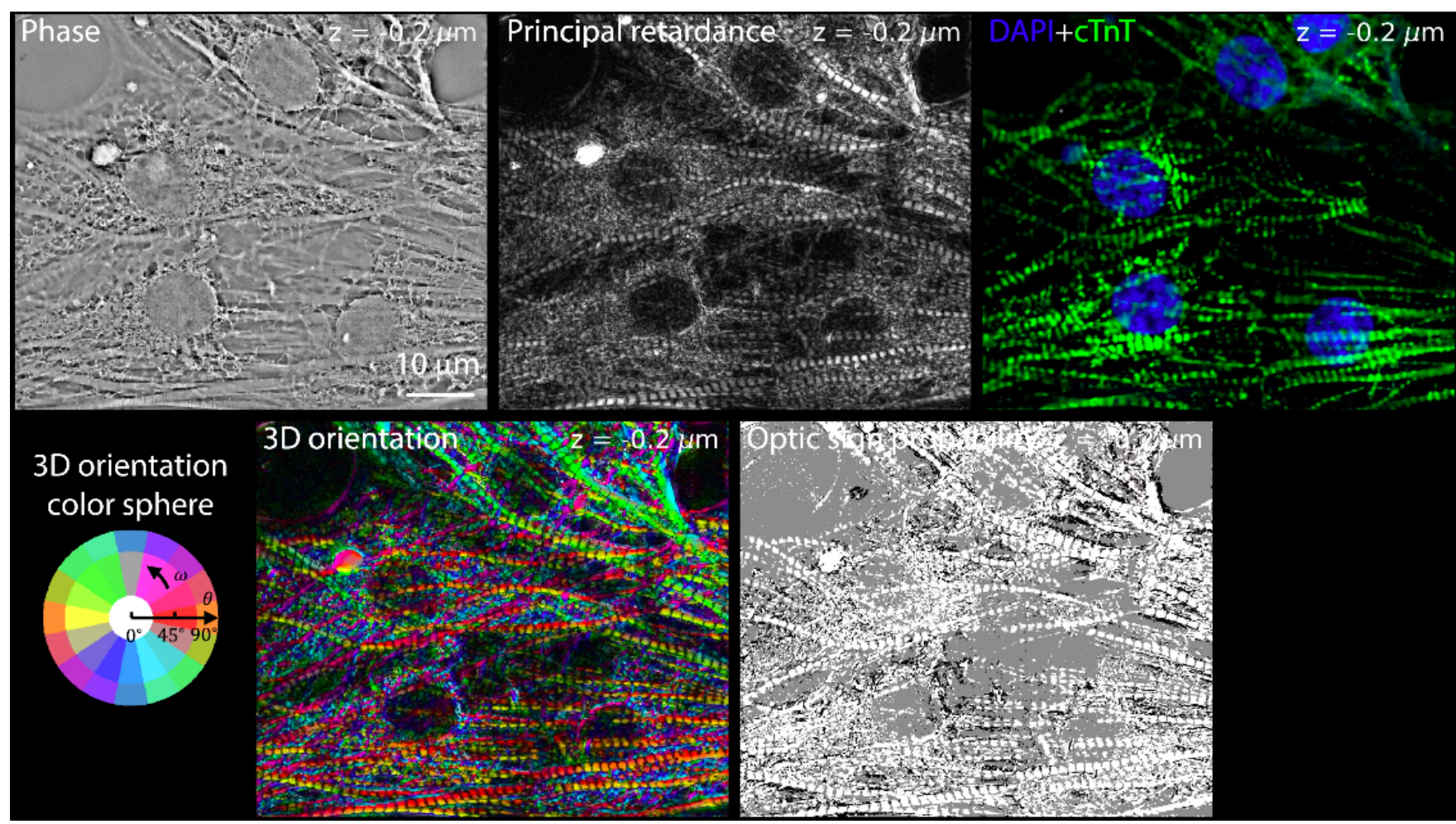

Video 7. $z$-stacks of phase, principal retardance, 3D orientation, optic sign probability, and fluorescence images of fixed healthy cardiomyocytes shown in fig. 5A.

\section{Supplementary Note 1: Derivation of the forward model}

3D distribution of optical phase and anisotropy of a specimen arise from the 3D distribution of the material's relative permittivity. This permittivity determines the amount of average and differential optical phase shift and the characteristic axes responding to different input polarization modes, which corresponds to specimens' density and anisotropy information. In the following subsections, we explain how the information of this permittivity is coupled to measurable quantities through vector wave equation $(71,72)$ and partially coherent imaging model $(73-75)$.

\section{A: Permittivity tensor}

Permittivity is a measure of how much a material is polarized by an applied electric field. The more easily a material is polarized, the slower a eletromagnetic wave is travelling in it, and more optically dense the material is. A polarized material radiates with a group of induced electric dipoles and creates measurable scattered fields. Understanding the permittivity of a material is key to extracting scattering-related optical quantities such as optical phase and anisotropy.

The permittivity is a scalar for an isotropic material such as water. The result of a scalar permittivity is that the induced electric dipole moment aligns and scales linearly with the applied electric field. In the case of an anisotropic material, the induced electric dipole moment still scales linearly with the applied electric field. However, its direction is dependent on the angle between the direction of the applied electric field and the orientation of the optic axis of the material. The permittivity of an anisotropic material hence takes a form of a tensor (3).

There are two types of anisotropic material depending on the number of optic axes they have, the uniaxial material and the biaxial material (3). Here we consider biological structure with at most one anisotropic axis (an uniaxial material). Assuming that the imaging system's optical axis is aligned along the $z$-axis as shown in fig. 1. We express the relative permittivity tensor of an uniaxial material oriented in the $z$-axis as

$$
\overline{\bar{\epsilon}}_{\mathrm{r}}=\left[\begin{array}{ccc}
n_{\mathrm{o}}^{2} & 0 & 0 \\
0 & n_{\mathrm{o}}^{2} & 0 \\
0 & 0 & n_{\mathrm{e}}^{2}
\end{array}\right]=\left[\begin{array}{ccc}
\epsilon_{\mathrm{r}}-\Delta \epsilon_{\mathrm{r}} & 0 & 0 \\
0 & \epsilon_{\mathrm{r}}-\Delta \epsilon_{\mathrm{r}} & 0 \\
0 & 0 & \epsilon_{\mathrm{r}}+\Delta \epsilon_{\mathrm{r}}
\end{array}\right],
$$

where $n_{\mathrm{o}}$ and $n_{\mathrm{e}}$ are refractive indices experienced by the ordinary and extraordinary wave, respectively. Refractive indices are generally complex numbers, whose real part causes phase delay and imaginary part introduces absorption. For the convenience of the following derivation, we express this permittivity tensor with the average permittivity $\left(\epsilon_{\mathrm{r}}\right)$ and the difference of 
permittivity $\left(\Delta \epsilon_{\mathrm{r}}\right)$ from the ordinary and etraordanary wave. They are related to the refractive indices as follows:

$$
\begin{aligned}
\epsilon_{\mathrm{r}} & =\frac{1}{2}\left(n_{\mathrm{e}}^{2}+n_{\mathrm{o}}^{2}\right) \\
\Delta \epsilon_{\mathrm{r}} & =\frac{1}{2}\left(n_{\mathrm{e}}^{2}-n_{\mathrm{o}}^{2}\right)
\end{aligned}
$$

To generally understand the induced dipole moment of an arbitrarily oriented uniaxial material, we consider the material oriented with in-plane orientation of $\omega$ and inclination of $\theta$ as shown in fig. 1B. The permittivity tensor in the microscope's frame of reference is obtained through applying rotational transformation on $\overline{\bar{\epsilon}}_{\mathrm{r}}$ and is expressed as (83)

$$
\begin{aligned}
\overline{\bar{\epsilon}}_{\mathrm{r}}(\omega, \theta) & =\overline{\bar{T}}(\omega, \theta)^{-1} \cdot \overline{\bar{\epsilon}}_{\mathrm{r}} \cdot \overline{\bar{T}}(\omega, \theta) \\
& =\left[\begin{array}{ccc}
\epsilon_{\mathrm{r}}-\Delta \epsilon_{\mathrm{r}}\left(\cos ^{2} \theta-\sin ^{2} \theta \cos 2 \omega\right) & \Delta \epsilon_{\mathrm{r}} \sin ^{2} \theta \sin 2 \omega & \Delta \epsilon_{\mathrm{r}} \sin 2 \theta \cos \omega \\
\Delta \epsilon_{\mathrm{r}} \sin ^{2} \theta \sin 2 \omega & \epsilon_{\mathrm{r}}-\Delta \epsilon_{\mathrm{r}}\left(\cos ^{2} \theta+\sin ^{2} \theta \cos 2 \omega\right) & \Delta \epsilon_{\mathrm{r}} \sin 2 \theta \sin \omega \\
\Delta \epsilon_{\mathrm{r}} \sin 2 \theta \cos \omega & \Delta \epsilon_{\mathrm{r}} \sin 2 \theta \sin \omega & \epsilon_{\mathrm{r}}+\Delta \epsilon_{\mathrm{r}} \cos 2 \theta
\end{array}\right]
\end{aligned}
$$

where $\overline{\bar{T}}(\omega, \theta)$ is the overall coordinate rotational matrix that is composed of two consecutive coordinate rotations around $z$ and $y$ axes as

$$
\overline{\bar{T}}(\omega, \theta)=\left[\begin{array}{ccc}
\cos \theta & 0 & -\sin \theta \\
0 & 1 & 0 \\
\sin \theta & 0 & \cos \theta
\end{array}\right] \cdot\left[\begin{array}{ccc}
\cos \omega & \sin \omega & 0 \\
-\sin \omega & \cos \omega & 0 \\
0 & 0 & 1
\end{array}\right]
$$

With this general form of permittivity, we will move to introduce how the induced dipole moment creates scattered electric fields that encode information of this permittivity tensor.

\section{B: Vector wave equation and Born approximation}

Following the scalar wave scattering theory (3), the core understanding of wave scattering event lies in the combined solution of homogeneous and inhomogeneous wave equation. In order to account for a more accurate description of the interaction between a polarized light and an anisotropic material, we start by considering the vector wave equation derived from Maxwell's equation as $(71,72)$

$$
\nabla \times \nabla \times \vec{E}(\vec{r})=k_{0}^{2} \overline{\bar{\epsilon}}_{\mathrm{r}}(\vec{r}) \vec{E}(\vec{r}),
$$

where $\vec{E}(\vec{r})=\left[E_{x}(\vec{r}), E_{y}(\vec{r}), E_{z}(\vec{r})\right]^{T}$ is the electric field in 3D space $\vec{r}=[x, y, z]^{T}, k_{0}=2 \pi / \lambda_{0}$ is the free-space wavenumber, and $\lambda_{0}$ is the free-space wavelength of the light. Our goal here is to find a general solution of the electric field in this equation. To solve this equation, we rewrite this equation as

$$
\nabla \times \nabla \times \vec{E}(\vec{r})-k_{0}^{2} \epsilon_{\mathrm{rm}} \vec{E}=k_{0}^{2}\left[\overline{\bar{\epsilon}}_{\mathrm{r}}(\vec{r})-\epsilon_{\mathrm{rm}}\right] \vec{E}(\vec{r})=\overline{\bar{f}}(\vec{r}) \vec{E}(\vec{r})
$$

where $\epsilon_{\mathrm{rm}}$ is the isotropic relative permittivity of the surrounding media and is a scalar value and $\overline{\bar{f}}(\vec{r})=k_{0}^{2}\left[\overline{\bar{\epsilon}}_{\mathrm{r}}(\vec{r})-\epsilon_{\mathrm{rm}}\right]$ is the scattering potential tensor. Setting the right-hand side of the Eq. (S6) to zero, we get a homogeneous solution $\vec{E}_{\text {inc }}(\vec{r})$, which can be simply an incident propagating plane wave. Treating the right-hand side of the Eq. (S6) as a source term, people have found its inhomogeneous solution as $(71,72)$

$$
\vec{E}_{\text {scat }}=\iiint \overline{\bar{G}}\left(\vec{r}-\vec{r}^{\prime}\right) \overline{\bar{f}}\left(\vec{r}^{\prime}\right) \vec{E}\left(\vec{r}^{\prime}\right) d^{3} \vec{r} .
$$

The $\overline{\bar{G}}(\vec{r})$ is the dyadic Green's tensor expressed as $(71,72)$

$$
\begin{gathered}
\overline{\bar{G}}(\vec{r})=\left[\begin{array}{lll}
G_{x x}(\vec{r}) & G_{x y}(\vec{r}) & G_{x z}(\vec{r}) \\
G_{y x}(\vec{r}) & G_{y y}(\vec{r}) & G_{y z}(\vec{r}) \\
G_{z x}(\vec{r}) & G_{z y}(\vec{r}) & G_{z z}(\vec{r})
\end{array}\right], \\
\text { where }\left\{\begin{array}{l}
G_{p q}(\vec{r})=\left(\delta_{p q}+\frac{\partial_{p} \partial_{q}}{\epsilon_{\mathrm{r}} k_{0}^{2}}\right) G(\vec{r}), \quad p, q=x, y, z \\
G(\vec{r})=\frac{e^{i \sqrt{\epsilon_{\mathrm{r}}} k_{0} r}}{4 \pi r}
\end{array}\right.
\end{gathered}
$$

Combining the homogeneous and inhomogenous solution, we get a solution in the following form

$$
\begin{aligned}
\vec{E}(\vec{r}) & =\vec{E}_{\mathrm{inc}}(\vec{r})+\vec{E}_{\mathrm{scat}}(\vec{r}) \\
& =\vec{E}_{\mathrm{inc}}(\vec{r})+\iiint \overline{\bar{G}}\left(\vec{r}-\vec{r}^{\prime}\right) \overline{\bar{f}}\left(\vec{r}^{\prime}\right) \vec{E}\left(\vec{r}^{\prime}\right) d^{3} \vec{r}
\end{aligned}
$$


The interaction term of scattering potential tensor $(\overline{\bar{f}}(\vec{r}))$ and the total electric field $(\vec{E}(\vec{r}))$ acts as an induced dipole moment radiating electric field to the space through the dyadic Green's tensor. This radiating field is the scattered field that is triggered by the incident electric field. The total field is sum of the incident and the scattered fields. However, this is not yet a solution, because the scattered field depends on the knowledge of the total electric field, which is a sum of the incident and scattered fields. A recursive approach $(84,85)$ is needed for a complete solution.

Rigorous approaches to solve the total electric field can be computationally intensive $(84,85)$. Here we consider biological specimens that are relatively weakly scattering. Hence, we are able to replace $\vec{E}(\vec{r})$ with $\vec{E}_{\text {inc }}(\vec{r})$ in Eq. (S7) by assuming the first Born approximation (3) and get

$$
\vec{E}(\vec{r}) \approx \vec{E}_{\mathrm{inc}}(\vec{r})+\iiint \overline{\bar{G}}\left(\vec{r}-\vec{r}^{\prime}\right) \overline{\bar{f}}\left(\vec{r}^{\prime}\right) \vec{E}_{\mathrm{inc}}\left(\vec{r}^{\prime}\right) d^{3} \vec{r} .
$$

This equation explains how the material is interacting with the incident electric field under weakly scattering condition.

\section{C: 3D vectorial partially coherent imaging model}

Here, we first establish the link from the scattered fields to measurable generalized Stokes parameters $(73,74)$ under partially coherent illumination. The relationship between the measurable Stokes parameters and the permittivity tensor (or scattering potential tensor) is generally nonlinear. In order to develop a more computationally efficient algorithm for the permittivity tensor retrieval, we sacrifice some accuracy by linearizing the model with the approach similar to $(26,34,35,86,87)$. We will show this linearization leads to handy transfer functions for further reconstruction of permittivity tensor. In the end, we follow this model with a reduction of dimensionality to consider 2D imaging case.

\section{C.1: Electric field of an incident plane wave}

Our polarization microscope measures Stokes parameters of scattered light from the specimen under quasi-monochromatic spatially partially coherent illumination in a Köhler geometry. Each point on the source plane (aperture plane of a illumination condenser lens) generates a plane wave with 3D spatial frequency of $\vec{\nu}=\left[\vec{\nu}_{\perp}^{T}, \nu_{z}\right]^{T}$, where $\vec{\nu}_{\perp}=\left[\nu_{x}, \nu_{y}\right]^{T}$ is the transverse spatial frequency and $\nu_{z}$ is the axial spatial frequency satisfying $\left|\vec{\nu}_{\perp}\right|^{2}+\nu_{z}^{2}=\frac{\epsilon_{\mathrm{rm}}}{\lambda_{0}^{2}}=\frac{1}{\lambda^{2}} . \lambda$ is the wavelength of light inside a media with permittivity of $\epsilon_{\mathrm{rm}}$. This plane wave is expressed as

$$
\vec{E}_{\text {inc }}\left(\vec{r}, \vec{\nu}_{\perp}\right)=\vec{E}_{0}\left(\vec{\nu}_{\perp}\right) \cdot e^{i 2 \pi \vec{\nu} \cdot \vec{r}}
$$

where $\vec{E}_{0}\left(\vec{\nu}_{\perp}\right)=\left[E_{0 x}\left(\vec{\nu}_{\perp}\right), E_{0 y}\left(\vec{\nu}_{\perp}\right), E_{0 z}\left(\vec{\nu}_{\perp}\right)\right]^{T}$ is the illumination-angle-dependent vector component of the incident plane wave from the lens-focusing effect.

Considering the polarization state of the light is known as $\left.\vec{E}_{s}\left(\vec{\nu}_{\perp}\right)=\left[E_{s x}\left(\vec{\nu}_{\perp}\right), E_{s y}\left(\vec{\nu}_{\perp}\right), 0\right)\right]$ in the source plane, which is usually the case experimentally, we relate it to the illumination-angle-dependent electric field of the incident wave through the lens focusing effect (88) as

$$
\begin{aligned}
& E_{0 x}\left(\vec{\nu}_{\perp}\right)=\frac{1}{\left\|\vec{\nu}_{\perp}\right\|_{2}^{2}} \cdot\left[E_{s x}\left(\vec{\nu}_{\perp}\right) \cdot\left(\nu_{x}^{2} \sqrt{1-\lambda^{2}\left\|\vec{\nu}_{\perp}\right\|_{2}^{2}}+\nu_{y}^{2}\right)+E_{s y}\left(\vec{\nu}_{\perp}\right) \cdot \nu_{x} \nu_{y}\left(\sqrt{1-\lambda^{2}\left\|_{\perp}\right\|_{2}^{2}}-1\right)\right] \\
& E_{0 y}\left(\vec{\nu}_{\perp}\right)=\frac{1}{\left\|\vec{\nu}_{\perp}\right\|_{2}^{2}} \cdot\left[E_{s x}\left(\vec{\nu}_{\perp}\right) \cdot \nu_{x} \nu_{y}\left(\sqrt{1-\lambda^{2}\left\|\vec{\nu}_{\perp}\right\|_{2}^{2}}-1\right)+E_{s y}\left(\vec{\nu}_{\perp}\right) \cdot\left(\nu_{y}^{2} \sqrt{1-\lambda^{2}\left\|\vec{\nu}_{\perp}\right\|_{2}^{2}}+\nu_{x}^{2}\right)\right] \\
& E_{0 z}\left(\vec{\nu}_{\perp}\right)=-\left[E_{s x}\left(\vec{\nu}_{\perp}\right) \cdot \lambda \nu_{x}+E_{s y}\left(\vec{\nu}_{\perp}\right) \cdot \lambda \nu_{y}\right]
\end{aligned}
$$

\section{C.2: Scattered field to generalized Stokes parameters}

For a Köhler geometry, each independent coherent plane wave coming from the source plane interacts with the specimen through Eq. (S10) to create scattered field and each $z$-plane of this 3D scattered field is convolved with a 2D coherent point spread function $h_{c}\left(\vec{r}_{\perp}\right)$ to form a 3D stack of output total field $\vec{E}_{\text {out }}(\vec{r})$. By expressing the scatter potential tensor as

$$
\overline{\bar{f}}(\vec{r})=\left[\begin{array}{lll}
f_{x x}(\vec{r}) & f_{x y}(\vec{r}) & f_{x z}(\vec{r}) \\
f_{y x}(\vec{r}) & f_{y y}(\vec{r}) & f_{y z}(\vec{r}) \\
f_{z x}(\vec{r}) & f_{z y}(\vec{r}) & f_{z z}(\vec{r})
\end{array}\right]
$$

we express one arbitrary component of $\vec{E}_{\text {out }}(\vec{r})$ as

$$
E_{\text {out }, p}\left(\vec{r}, \vec{\nu}_{\perp}\right)=\left\{E_{0 p}\left(\vec{\nu}_{\perp}\right) \cdot e^{i 2 \pi \vec{\nu} \cdot \vec{r}}+\sum_{q=x, y, z} G_{p q}(\vec{r}) \otimes\left[\left(\sum_{\ell=x, y, z} f_{q \ell}(\vec{r}) E_{0 \ell}\left(\overrightarrow{\nu_{\perp}}\right)\right) \cdot e^{i 2 \pi \vec{\nu} \cdot \vec{r}}\right]\right\} \otimes h_{c}\left(\vec{r}_{\perp}\right) \delta(z),(\text { S14) }
$$

where $p=x, y, z, \otimes$ denotes $3 \mathrm{D}$ convolution, and $\vec{r}_{\perp}=[x, y]^{T}$ is the transverse coordinate of $\vec{r}$. 
We have so far derived the scattered electric field on our detector from a single plane wave in a partially coherent source pattern. Different partially coherent source patterns $(\alpha)$ are specified by different sets of $\vec{\nu}_{\perp}$. By integrating the contribution of all the coherent scattered modes from one illumination pattern, we obtain the general Stokes parameters $(73,74)$ as

$$
\left[\begin{array}{c}
S_{0, \alpha}(\vec{r}) \\
S_{1, \alpha}(\vec{r}) \\
S_{2, \alpha}(\vec{r}) \\
S_{3, \alpha}(\vec{r})
\end{array}\right]=\left[\begin{array}{c}
S_{x x, \alpha}(\vec{r})+S_{y y, \alpha}(\vec{r}) \\
S_{x x, \alpha}(\vec{r})-S_{y y, \alpha}(\vec{r}) \\
S_{x y, \alpha}(\vec{r})+S_{y x, \alpha}(\vec{r}) \\
i\left[S_{x y, \alpha}(\vec{r})-S_{y x, \alpha}(\vec{r})\right]
\end{array}\right],
$$

$$
\text { where } \quad S_{p q, \alpha}(\vec{r})=\iint_{\vec{\nu}_{\perp} \in \alpha} E_{\text {out }, p}\left(\vec{r}, \vec{\nu}_{\perp}\right) E_{\text {out }, q}^{*}\left(\vec{r}, \vec{\nu}_{\perp}\right) d^{2} \vec{\nu}_{\perp} \text {. }
$$

For ease of the following analysis, we take a closer look at the Fourier space of $S_{p q, \alpha}(\vec{r})$ as

$$
\begin{aligned}
\tilde{S}_{p q, \alpha}(\vec{u})= & \iint_{\vec{\nu}_{\perp} \in \alpha} E_{\text {out }, p}\left(\vec{u}, \vec{\nu}_{\perp}\right) \otimes E_{\text {out }, q}^{*}\left(-\vec{u}, \vec{\nu}_{\perp}\right) d^{2} \vec{\nu}_{\perp} \\
= & \iint_{\vec{\nu}_{\perp} \in \alpha} d^{2} \vec{\nu}_{\perp}\left\{\left[E_{0 p}\left(\vec{\nu}_{\perp}\right) \delta(\vec{u}-\vec{\nu})+\sum_{\beta=x, y, z} \tilde{G}_{p \beta}(\vec{u}) \cdot\left(\sum_{\gamma=x, y, z} \tilde{f}_{\beta \gamma}(\vec{u}-\vec{\nu}) E_{0 \gamma}\left(\overrightarrow{\nu_{\perp}}\right)\right)\right] \cdot \tilde{h}_{c}\left(\vec{u}_{\perp}\right)\right\} \\
& \otimes\left\{\left[E_{0 q}^{*}\left(\vec{\nu}_{\perp}\right) \delta(-\vec{u}-\vec{\nu})+\sum_{\xi=x, y, z} \tilde{G}_{q \xi}^{*}(-\vec{u}) \cdot\left(\sum_{\eta=x, y, z} \tilde{f}_{\xi \eta}^{*}(-\vec{u}-\vec{\nu}) E_{0 \eta}^{*}\left(\overrightarrow{\nu_{\perp}}\right)\right)\right] \cdot \tilde{h}_{c}^{*}\left(-\vec{u}_{\perp}\right)\right\}
\end{aligned}
$$

where $\tilde{a}(\vec{u})$ denotes the Fourier transform of a function $a(\vec{r})$ at the 3D spatial frequency $\vec{u}=\left[\vec{u}_{\perp}^{T}, u_{z}\right]^{T}$. From this equation, we observe the measurable Stokes components, $\tilde{S}_{p q, \alpha}(\vec{u})$, are nonlinearly related to our targeted information, $\tilde{f}_{p q}(\vec{u})$. To solve this rigorously, it requires an iterative optimization algorithm as in the classic phase retrieval problem (89), which can be computationally heavy (time consuming) and subject to convergence instability (90). Instead, we adopt the linearization approach to simplify this equation for simpler, faster, and more robust solution.

\section{C.3: Linearization of the model}

Following previous section, we expand Eq. (S16) further into three major contribution terms

$$
\begin{aligned}
& \tilde{S}_{p q, \alpha}(\vec{u})=\tilde{S}_{p q, \alpha, \mathrm{DC}}(\vec{u})+\tilde{S}_{p q, \alpha, \mathrm{L}}(\vec{u})+\tilde{S}_{p q, \alpha, \mathrm{NL}}(\vec{u}), \\
& \text { where }\left\{\begin{array}{c}
\tilde{S}_{p q, \alpha, \mathrm{DC}}(\vec{u})=\left[\iint_{\vec{\nu}_{\perp} \in \alpha} d^{2} \vec{\nu}_{\perp} \cdot E_{0 p}\left(\vec{\nu}_{\perp}\right) E_{0 q}^{*}\left(\vec{\nu}_{\perp}\right)\left|\tilde{h}_{c}\left(\vec{\nu}_{\perp}\right)\right|^{2}\right] \cdot \delta(\vec{u}) \\
\tilde{S}_{p q, \alpha, \mathrm{L}}(\vec{u})=\sum_{\substack{\beta=x, y, z \\
\gamma=x, y, z}} \iint_{\vec{\nu}_{\perp} \in \alpha} d^{2} \vec{\nu}_{\perp} \cdot E_{0 q}^{*}\left(\vec{\nu}_{\perp}\right) E_{0 \gamma}\left(\vec{\nu}_{\perp}\right) \tilde{h}_{c}^{*}\left(\vec{\nu}_{\perp}\right) \tilde{G}_{p \beta}(\vec{u}+\vec{\nu}) \tilde{h}_{c}\left(\vec{u}_{\perp}+\vec{\nu}_{\perp}\right) \tilde{f}_{\beta \gamma}(\vec{u}) \\
+\sum_{\substack{\xi=x, y, z \\
\eta=x, y, z}} \iint_{\vec{\nu}_{\perp} \in \alpha} d^{2} \vec{\nu}_{\perp} \cdot E_{0 p}\left(\vec{\nu}_{\perp}\right) E_{0 \eta}^{*}\left(\vec{\nu}_{\perp}\right) \tilde{h}_{c}\left(\vec{\nu}_{\perp}\right) \tilde{G}_{q \xi}^{*}(-\vec{u}+\vec{\nu}) \tilde{h}_{c}^{*}\left(-\vec{u}_{\perp}+\vec{\nu}_{\perp}\right) \tilde{f}_{\xi \eta}^{*}(-\vec{u}) \\
\tilde{S}_{p q, \alpha, \mathrm{NL}}(\vec{u})=\sum_{\substack{\beta=x, y, z \xi=x, y, z \\
\gamma=x, y, z}} \iint_{\vec{\nu}_{\perp} \in \alpha} d^{2} \vec{\nu}_{\perp} \cdot\left[\tilde{G}_{p \beta}(\vec{u}) \tilde{h}_{c}\left(\vec{u}_{\perp}\right) \tilde{f}_{\beta \gamma}(\vec{u}-\vec{\nu}) E_{0 \gamma}\left(\vec{\nu}_{\perp}\right)\right] \\
\otimes\left[\tilde{G}_{q \xi}^{*}(-\vec{u}) \tilde{h}_{c}^{*}\left(-\vec{u}_{\perp}\right) \tilde{f}_{\xi \eta}(-\vec{u}-\vec{\nu}) E_{0 \eta}^{*}\left(\overrightarrow{\nu_{\perp}}\right)\right]
\end{array}\right.
\end{aligned}
$$

$\tilde{S}_{p q, \alpha, \mathrm{DC}}$ is the DC component of a Stokes component, $\tilde{S}_{p q, \alpha}(\vec{u})$, that has the strongest energy that comes from the incident electric field. $\tilde{S}_{p q, \alpha, \mathrm{L}}$ is the term that the scattering potential tensor are linearly related to the Stokes component. This is the second dominant term and it comes from the interference of the scattered electric field and the incident electric field. $\tilde{S}_{p q, \alpha, \mathrm{NL}}$ comes from interference between scattered fields, which is the weakest among three, and are nonlinearly related to the Stokes component.

In transparent biological specimen, most of the signals in the Stokes parameters come from the DC and the linear terms. We hence assume the contribution from the scattered field interference is negligible. Neglecting the nonlinear term and subtracting the DC term from $\tilde{S}_{p q, \alpha}(\vec{u})$, we deal only with the linear term as

$$
\tilde{S}_{p q, \alpha}^{\prime}(\vec{u})=\tilde{S}_{p q, \alpha}(\vec{u})-\tilde{S}_{p q, \alpha, \mathrm{DC}}(\vec{u}) \approx \tilde{S}_{p q, \alpha, \mathrm{L}}(\vec{u})
$$

After this linearization, we see the DC-substracted Stokes component is a sum of filtered scattering potential tensor components, $f_{\beta \gamma}(\vec{u})$ and $f_{\xi \eta}^{*}(-\vec{u})$, as seen in Eq. (S17). These corresponding filters are essentially weak object transfer functions presented 
in the scalar wave scattering theory $(26,34,35,86,87)$. We introduce a set of notations to simplify their expressions as

$$
\begin{aligned}
\mathcal{M}_{+}\left[E_{0 q}^{*}, E_{0 \gamma}, \tilde{G}_{p \beta}\right] & =\iint_{\vec{\nu}_{\perp} \in \alpha} d^{2} \vec{\nu}_{\perp} \cdot E_{0 q}^{*}\left(\vec{\nu}_{\perp}\right) E_{0 \gamma}\left(\overrightarrow{\nu_{\perp}}\right) \tilde{h}_{c}^{*}\left(\vec{\nu}_{\perp}\right) \tilde{G}_{p \beta}(\vec{u}+\vec{\nu}) \tilde{h}_{c}\left(\vec{u}_{\perp}+\vec{\nu}_{\perp}\right) \\
\mathcal{M}_{-}\left[E_{0 p}, E_{0 \eta}^{*}, \tilde{G}_{q \xi}^{*}\right] & =\iint_{\vec{\nu}_{\perp} \in \alpha} d^{2} \vec{\nu}_{\perp} \cdot E_{0 p}\left(\vec{\nu}_{\perp}\right) E_{0 \eta}^{*}\left(\overrightarrow{\nu_{\perp}}\right) \tilde{h}_{c}\left(\vec{\nu}_{\perp}\right) \tilde{G}_{q \xi}^{*}(-\vec{u}+\vec{\nu}) \tilde{h}_{c}^{*}\left(-\vec{u}_{\perp}+\vec{\nu}_{\perp}\right),
\end{aligned}
$$

where $\mathcal{M}_{+}$and $\mathcal{M}_{-}$are mappings from the associated incident electric field components and dyadic Green functions to the actual transfer functions.

To relate the Stokes parameters to more physical interpretable quantities from the scattering potential tensor, we decompose the tensor components into the following forms

$$
\begin{aligned}
& f_{x x}(\vec{r})=f_{0}(\vec{r})+f_{1 \mathrm{c}}(\vec{r}), \quad f_{y y}(\vec{r})=f_{0}(\vec{r})-f_{1 \mathrm{c}}(\vec{r}), \quad f_{z z}(\vec{r})=f_{0}(\vec{r})+f_{3}(\vec{r}), \\
& f_{x y}(\vec{r})=f_{y x}(\vec{r})=f_{1 \mathrm{~s}}(\vec{r}), \quad f_{y z}(\vec{r})=f_{z y}(\vec{r})=f_{2 \mathrm{~s}}(\vec{r}), \quad f_{z x}(\vec{r})=f_{x z}(\vec{r})=f_{2 \mathrm{c}}(\vec{r}), \\
& \text { where } \quad\left\{\begin{array}{l}
f_{0}(\vec{r})=k_{0}^{2}\left[\epsilon_{\mathrm{r}}(\vec{r})-\epsilon_{\mathrm{rm}}-\Delta \epsilon_{\mathrm{r}}(\vec{r}) \cdot \cos ^{2} \theta(\vec{r})\right] \\
f_{1 \mathrm{c}}(\vec{r})=k_{0}^{2} \Delta \epsilon_{\mathrm{r}}(\vec{r}) \cdot \sin ^{2} \theta(\vec{r}) \cdot \cos 2 \omega(\vec{r}) \\
f_{1 \mathrm{~s}}(\vec{r})=k_{0}^{2} \Delta \epsilon_{\mathrm{r}}(\vec{r}) \cdot \sin ^{2} \theta(\vec{r}) \cdot \sin 2 \omega(\vec{r}) \\
f_{2 \mathrm{c}}(\vec{r})=k_{0}^{2} \Delta \epsilon_{\mathrm{r}}(\vec{r}) \cdot \sin ^{2} 2 \theta(\vec{r}) \cdot \cos \omega(\vec{r}) \\
f_{2 \mathrm{~s}}(\vec{r})=k_{0}^{2} \Delta \epsilon_{\mathrm{r}}(\vec{r}) \cdot \sin 2 \theta(\vec{r}) \cdot \sin \omega(\vec{r}) \\
f_{3}(\vec{r})=k_{0}^{2} \Delta \epsilon_{\mathrm{r}}(\vec{r})\left[3 \cos ^{2} \theta(\vec{r})-1\right]
\end{array}\right.
\end{aligned}
$$

Here we assume $f_{0}(\vec{r})=f_{0 \mathrm{r}}(\vec{r})+i f_{0 \mathrm{i}}(\vec{r})$ is a complex function carrying density (average refractive indices) information of the material $\left(\operatorname{Re}\left\{\epsilon_{\mathrm{r}}(\vec{r})-\epsilon_{\mathrm{rm}}\right\}\right)$ in the real part and the absorption information of the material $\left(\operatorname{Im}\left\{\epsilon_{\mathrm{r}}(\vec{r})-\epsilon_{\mathrm{rm}}\right\}\right)$ in the imaginary part. $f_{1 \mathrm{c}}(\vec{r}), f_{1 \mathrm{~s}}(\vec{r}), f_{2 \mathrm{c}}(\vec{r}), f_{2 \mathrm{~s}}(\vec{r})$, and $f_{3}(\vec{r})$ are assumed to be real functions (ignoring the effect of diattenuation), providing information of anisotropy $\left(\Delta \epsilon_{\mathrm{r}}(\vec{r})\right)$, in-plane orientation $(\omega(\vec{r}))$, and inclination $(\theta(\vec{r}))$.

By grouping all the linear contribution from the same tensor component in Eq. (S17) together, we arrive at this 7-unknownvariable linear equation to solve for

$$
\begin{aligned}
\tilde{S}_{p q, \alpha}^{\prime}(\vec{u})= & \tilde{f}_{0 \mathrm{r}}(\vec{u}) \cdot\left\{\sum_{\beta=x, y, z}\left(\mathcal{M}_{+}\left[E_{0 q}^{*}, E_{0 \beta}, \tilde{G}_{p \beta}\right]+\mathcal{M}_{-}\left[E_{0 p}, E_{0 \beta}^{*}, \tilde{G}_{q \beta}^{*}\right]\right)\right\} \\
& +\tilde{f}_{0 \mathrm{i}}(\vec{u}) \cdot i\left\{\sum_{\beta=x, y, z}\left(\mathcal{M}_{+}\left[E_{0 q}^{*}, E_{0 \beta}, \tilde{G}_{p \beta}\right]-\mathcal{M}_{-}\left[E_{0 p}, E_{0 \beta}^{*}, \tilde{G}_{q \beta}^{*}\right]\right)\right\} \\
& +\tilde{f}_{1 \mathrm{c}}(\vec{u}) \cdot\left\{\mathcal{M}_{+}\left[E_{0 q}^{*}, E_{0 x}, \tilde{G}_{p x}\right]-\mathcal{M}_{+}\left[E_{0 q}^{*}, E_{0 y}, \tilde{G}_{p y}\right]+\mathcal{M}_{-}\left[E_{0 p}, E_{0 x}^{*}, \tilde{G}_{q x}^{*}\right]-\mathcal{M}_{-}\left[E_{0 p}, E_{0 y}^{*}, \tilde{G}_{q y}^{*}\right]\right\} \\
& +\tilde{f}_{1 \mathrm{~s}}(\vec{u}) \cdot\left\{\mathcal{M}_{+}\left[E_{0 q}^{*}, E_{0 y}, \tilde{G}_{p x}\right]+\mathcal{M}_{+}\left[E_{0 q}^{*}, E_{0 x}, \tilde{G}_{p y}\right]+\mathcal{M}_{-}\left[E_{0 p}, E_{0 y}^{*}, \tilde{G}_{q x}^{*}\right]+\mathcal{M}_{-}\left[E_{0 p}, E_{0 x}^{*}, \tilde{G}_{q y}^{*}\right]\right\} \\
& +\tilde{f}_{2 \mathrm{c}}(\vec{u}) \cdot\left\{\mathcal{M}_{+}\left[E_{0 q}^{*}, E_{0 z}, \tilde{G}_{p x}\right]+\mathcal{M}_{+}\left[E_{0 q}^{*}, E_{0 x}, \tilde{G}_{p z}\right]+\mathcal{M}_{-}\left[E_{0 p}, E_{0 z}^{*}, \tilde{G}_{q x}^{*}\right]+\mathcal{M}_{-}\left[E_{0 p}, E_{0 x}^{*}, \tilde{G}_{q z}^{*}\right]\right\} \\
& +\tilde{f}_{2 \mathrm{~s}}(\vec{u}) \cdot\left\{\mathcal{M}_{+}\left[E_{0 q}^{*}, E_{0 z}, \tilde{G}_{p y}\right]+\mathcal{M}_{+}\left[E_{0 q}^{*}, E_{0 y}, \tilde{G}_{p z}\right]+\mathcal{M}_{-}\left[E_{0 p}, E_{0 z}^{*}, \tilde{G}_{q y}^{*}\right]+\mathcal{M}_{-}\left[E_{0 p}, E_{0 y}^{*}, \tilde{G}_{q z}^{*}\right]\right\} \\
& +\tilde{f}_{3}(\vec{u}) \cdot\left\{\mathcal{M}_{+}\left[E_{0 q}^{*}, E_{0 z}, \tilde{G}_{p z}\right]+\mathcal{M}_{-}\left[E_{0 p}, E_{0 z}^{*}, \tilde{G}_{q z}^{*}\right]\right\}
\end{aligned}
$$

Each scattering potential tensor component has its transfer function to link to the Stokes component. However, the actual Stokes parameters in Eq. (S15) are a sum or a difference of the Stokes components. By merging all the contribution once again for $p, q=x, y$, we summarize our linearization of the Stokes parameters to scattering potential tensor in one equation as

$$
\tilde{S}_{m, \alpha}^{\prime}(\vec{u})=\sum_{\substack{\ell=0 \mathrm{r}, 0 \mathrm{i}, 1 \mathrm{c}, 1 \mathrm{~s}, 2 \mathrm{c}, 2 \mathrm{~s}, 3}} \tilde{H}_{m, \ell, \alpha}(\vec{u}) \tilde{f}_{\ell}(\vec{u}) ; \quad m=0,1,2,3,
$$

where $\tilde{S}_{m, \alpha}^{\prime}(\vec{u})$ is the DC-subtracted Stokes parameter and $\tilde{H}_{m, \ell, \alpha}(\vec{u})$ is the transfer function mapping from each tensor component $\ell$ to the $m$-th Stokes parameter under illumination pattern $\alpha$. A detailed expression for these transfer functions are shown in the Appendix.

\section{C.4: Reduction to $2 D$ imaging model}

When the thickness of a specimen is smaller than the depth of field of a certain imaging condition, acquiring data at one plane by changing the partially coherent illumination patterns enables extraction of $2 \mathrm{D}$ phase and anisotropic information at that 
plane. This $2 \mathrm{D}$ information extraction requires a slight modification of the transfer functions. In this $2 \mathrm{D}$ imaging case, we consider the scattering potential tensor to be concentrated in a single axial layer and is expressed as

$$
\overline{\bar{f}}(\vec{r})=\left[\int_{z \in \mathrm{DOF}} \overline{\bar{f}}(\vec{r}) \cdot d z\right] \cdot \delta(z)=\overline{\bar{g}}\left(\vec{r}_{\perp}\right) \cdot \delta(z),
$$

where $\overline{\bar{g}}\left(\vec{r}_{\perp}\right)$ is the scattering potential tensor integrated in the $z$-direction over depth of field. We then substitute this expression for $\tilde{f}_{\ell}(\vec{u})$ in Eq. (S22) and integrated in $u_{z}$-direction to get a corresponding relationship in the 2D imaging case between 2D Stokes parameters and the integrated scattering potential tensor as

$$
\tilde{S}_{m, \alpha}^{\prime}\left(\vec{u}_{\perp}, z=0\right)=\int \tilde{S}_{m, \alpha}^{\prime}(\vec{u}) \cdot d u_{z}=\sum_{\substack{\ell=0 \mathrm{r}, 0 \mathrm{i}, 1 \mathrm{c}, 1 \mathrm{~s}, 2 \mathrm{c}, 2 \mathrm{~s}, 3}}\left[\int \tilde{H}_{m, \ell, \alpha}(\vec{u}) \cdot d u_{z}\right] \cdot \tilde{g}_{\ell}\left(\vec{u}_{\perp}\right) ; \quad m=0,1,2,3,
$$

The transfer functions in 2D imaging case are simply a $u_{z}$-integration of the previously established 3D transfer functions.

\section{Supplementary Note 2: Derivation of the Stokes background correction}

Since background variation is usually slowly varying, we adopt a non-diffraction-based Mueller calculus to develop our background removal scheme. An anisotropic sample with transmittance $t_{s}=\left|e^{\mu+i \phi}\right|^{2}$, in-plane orientation of $\omega$, out-of-plane orientation of $\theta$, apparent retardance $\rho_{\text {ap }}(\omega, \theta)$ can be modeled with an Mueller matrix as

$$
\overline{\bar{M}}_{s}\left(t_{s}, \omega, \theta, \rho_{\mathrm{ap}}\right) \approx t_{s}\left[\begin{array}{cccc}
1 & 0 & 0 & 0 \\
0 & 1 & 0 & -\sin 2 \omega \cdot \rho_{\mathrm{ap}}(\omega, \theta) \\
0 & 0 & 1 & \cos 2 \omega \cdot \rho_{\mathrm{ap}}(\omega, \theta) \\
0 & \sin 2 \omega \cdot \rho_{\mathrm{ap}}(\omega, \theta) & -\cos 2 \omega \cdot \rho_{\mathrm{ap}}(\omega, \theta) & 1
\end{array}\right] \text {, when } \rho_{\mathrm{ap}}(\omega, \theta) \ll 1 \text { (S25) }
$$

Sending in the circularly polarized light with the Stokes parameter of $\vec{S}_{s}=S_{s 0} \cdot[1,0,0,1]^{T}$, where $S_{s 0}$ is the energy of the incident light, the output Stokes parameters are obtained as

$$
\vec{S}_{s, \text { out }}=\bar{M}_{s}\left(t_{s}, \omega, \theta, \rho_{\mathrm{ap}}\right) \cdot \vec{S}_{s}=S_{s 0} \cdot\left[\begin{array}{c}
t_{s} \\
-t_{s} \cdot \sin 2 \omega \cdot \rho_{\mathrm{ap}}(\omega, \theta) \\
t_{s} \cdot \cos 2 \omega \cdot \rho_{\mathrm{ap}}(\omega, \theta) \\
t_{s}
\end{array}\right]
$$

This is the Stokes parameters we get assuming we do not have extra background polarization effects from the imaging optics in the microscope. When the imaging optics are not perfect, slowly varying polarization modulation appears to mask the weak anisotropy signal out. To find a way to remove this effect, we model the background polarization effect to be an additional transparent retarder with the Mueller matrix of $\overline{\bar{M}}_{\mathrm{bg}}\left(t_{\mathrm{bg}}, \omega_{\mathrm{bg}}, \theta_{\mathrm{bg}}, \rho_{\mathrm{bg}}\right)$. With this background Mueller matrix, the overall output Stokes parameters are obtained as

$$
\begin{aligned}
\vec{S}_{\text {out }}=\left[\begin{array}{l}
S_{\text {out }, 0} \\
S_{\text {out }, 1} \\
S_{\text {out }, 2} \\
S_{\text {out }, 3}
\end{array}\right] & =\overline{\bar{M}}_{\mathrm{bg}}\left(t_{\mathrm{bg},}, \omega_{\mathrm{bg}}, \theta_{\mathrm{bg}}, \rho_{\mathrm{bg}}\right) \cdot \overline{\bar{M}}_{s}\left(t_{s}, \omega, \theta, \rho_{\mathrm{ap}}\right) \cdot \vec{S}_{s} \\
& \approx t_{\mathrm{bg}} \cdot \vec{S}_{s, \text { out }}+S_{s 0} \cdot\left[\begin{array}{c}
0 \\
-t_{s} \cdot t_{\mathrm{bg}} \cdot \sin 2 \omega_{\mathrm{bg}} \cdot \rho_{\mathrm{bg}} \\
t_{s} \cdot t_{\mathrm{bg}} \cdot \cos 2 \omega_{\mathrm{bg}} \cdot \rho_{\mathrm{bg}} \\
0
\end{array}\right]
\end{aligned}
$$

This overall Stokes parameters contain contributions from the specimen and the background effect. To separate these effects, we acquire the background Stokes parameters by imaging an area without the specimen and we get

$$
\vec{S}_{\mathrm{bg}}=\left[\begin{array}{c}
S_{\mathrm{bg}, 0} \\
S_{\mathrm{bg}, 1} \\
S_{\mathrm{bg}, 2} \\
S_{\mathrm{bg}, 3}
\end{array}\right]=\overline{\bar{M}}_{\mathrm{bg}}\left(t_{\mathrm{bg}}, \omega_{\mathrm{bg}}, \theta_{\mathrm{bg}}, \rho_{\mathrm{bg}}\right) \cdot \vec{S}_{s}=S_{s 0} \cdot\left[\begin{array}{c}
t_{\mathrm{bg}} \\
-t_{\mathrm{bg}} \cdot \sin 2 \omega_{\mathrm{bg}} \cdot \rho_{\mathrm{bg}} \\
t_{\mathrm{bg}} \cdot \cos 2 \omega_{\mathrm{bg}} \cdot \rho_{\mathrm{bg}} \\
t_{\mathrm{bg}}
\end{array}\right]
$$

Comparing Eq. (S27) with Eq. (S28), we then come up with a correction to get the corrected Stokes parameters as

$$
\vec{S}_{c}=\frac{\vec{S}_{s, \mathrm{out}}}{S_{s 0}}=\frac{\vec{S}_{\mathrm{out}}}{S_{\mathrm{bg}, 0}}-\left[\begin{array}{c}
0 \\
S_{\mathrm{bg}, 1} \\
S_{\mathrm{bg}, 2} \\
0
\end{array}\right] \cdot \frac{S_{\mathrm{out}, 0}}{S_{\mathrm{bg}, 0}^{2}}
$$


By doing this correction, we also remove the DC components of the Stokes parameters in $S_{\text {out }, 1}$ and $S_{\text {out }, 2}$. The only remaining Stokes parameters with the DC-component is the $S_{0}$ component of $\vec{S}_{c}$. With the additional subtraction, we arrive at the final DC-subtracted Stokes parameters that we used for the deconvolution

$$
\vec{S}^{\prime}=\vec{S}_{c}-\left[\begin{array}{l}
1 \\
0 \\
0 \\
1
\end{array}\right]
$$

We apply these corrections on all sets of intensities from each illumination patterns before the deconvolution algorithm.

\section{Supplementary Note 3: The formation of laser-induced modifications in fused silica}

When an intense femtosecond laser pulse is focused into transparent material, e. g. silica glass, high-order nonlinear absorption allows the energy to be deposited predominantly within the focal volume, producing a local permanent refractive-index modification. Although the process of energy absorption is now well understood, the actual formation of the directly written structures is still controversial.

Depending on the level of laser intensity, one can induce any of four qualitatively different types of material modification: positive refractive-index change (type 1) at relatively low intensity; birefringent regions with the evidence of nanograting formation (type 2) $(18,50)$ at intermediate intensity and voids (type 3 ) at high intensity. The transition threshold from one kind of structure to another depends on the laser writing parameters such as pulse duration, wavelength and irradiation time. The nanograting type (type 2) of modifications is observed under SEM as periodic subwavelength arrangement of nanoplanes with features as small $20 \mathrm{~nm}$. Recently new type of birefringent modification has been observed as random arrangement of prolate-shape nanopores at transition from the type 1 to type 2 and coined as type X modification (19). The orientation of both nanogratings and elongated nanopores can be controlled by the polarization of the writing laser and results in form birefringence with slow axis oriented perpendicular to the polarization of writing laser and along the nanoplanes or long axis of prolate-shape nanopores. The induced birefringence is negative with the value for type 2 close to the birefringence in quartz crystal, and for type $\mathrm{X}$ of about 4 times lower value.

Birefringent structures produced with type $\mathrm{X}$ modification reveal ultra- low scattering loss and were observed when writing with infrared $1030 \mathrm{~nm}$ laser wavelength. Writing with shorter wavelength, such as $515 \mathrm{~nm}$ is advantageous for imprinting with high spatial resolution. However, only type 2 birefringent structures were revealed with the Abrio birefringence imaging system, which can only measure the average change of birefringence along axial direction. The structure of imprinted birefringent modification, e. g. the possible coexistence of different types of modification along the axial direction shown in fig. 2C, in principle can be characterized by SEM. However non-destructive method of 3D birefringence imaging, such as uPTI, is more convenient and resulted in the observation of type $\mathrm{X}$ structures in the tail of birefringent modification. This observation allows improving resolution of low loss birefringent structuring in silica glass. 